\title{
Estados Coerentes: O Grupo Simplético e Generalizações
}

\author{
Marcel Novaes
}

Tese apresentada ao Instituto de Física de São Carlos, da Universidade de São Paulo, para obtenção do título de Doutor em Ciências: Física Básica.

Orientador: Prof. Dr. José Eduardo Martinho Hornos

São Carlos 2003 
Novaes, Marcel

"Estados Coerentes: O Grupo Simplético e Generalizações"

Marcel Novaes - São Carlos, 2003.

Tese (Doutorado)-Instituto de Física de São Carlos

Universidade de São Paulo, 2003 - 128 páginas.

Orientador: Prof. Dr. José Eduardo Martinho Hornos

1. Estados Coerentes. 2. Álgebras de Lie 3. Métodos Semiclássicos. I. Título. 


\section{Agradecimentos}

- Ao Prof. José Eduardo Hornos, por ter aceito orientar este trabalho, por têlo feito com empenho e pelo clima de liberdade intelectual que me propiciou

- Ao Prof. Esmerindo Bernardes, presença indispensável em diversas etapas, sempre amigo e prestativo

- Ao Prof. Jean-Pierre Gazeau e o pessoal do LPTMC-Paris 7, pela hospitalidade

- Aos Profs. Marcus Aguiar, John Klauder, Sven Gnutzmann, Syed Ali, Reginaldo Napolitano e Miled Moussa, por todas as discussões que tiveram comigo ao longo do trabalho

- A todos os meus queridos amigos e amigas; sem eles a vida seria muito difícil

- Aos meus pais, Celso e Regina, e aos meus irmãos, Paulinha e Rica, por estarem sempre ao meu lado

- À Alê, essencial, por tudo, principalmente pela paciência

- A Salinger, Bukowski, Dylan, Leminski, Viana, Russo, Mann, Hesse e tantos outros; aos meus antigos professores, em particular na Oca; aos funcionários do Instituto de Física, especialmente os da biblioteca.

- A todos aqueles que esqueci de mencionar

Esta Tese é dedicada aos meus pais 
Este trabalho teve o apoio financeiro da Fundação de Amparo à Pesquisa do Estado de São Paulo (FAPESP). 


\author{
Ô Nêga \\ O que é que tem no balaio? \\ Nêga, Nêga Nêga \\ O que é que tem nesse balaio? \\ No balaio tem de tudo \\ Que alegra o coração \\ Divise o seu conteúdo \\ E fique quase mudo \\ De estupefação \\ Mas precisa ser sensível \\ Se quiser apreciar \\ Fica tudo invisível \\ A quem não sabe sonhar
}

Ouviste, Nêgo?

Itamar Assumpção, Balaio, 1994 


\section{Conteúdo}

1 Introdução 12

2 Estados Coerentes $\quad 17$

2.1 Oscilador Harmônico . . . . . . . . . . . . . . . . . . . . . . 17

2.1.1 Definição e Propriedades . . . . . . . . . . . . . . . 17

2.1.2 Integral de trajetória . . . . . . . . . . . . . . . . 20

2.1.3 Limite Semiclássico . . . . . . . . . . . . . . . . . . 22

2.2 Momentum Angular . . . . . . . . . . . . . . . . . . . . . . 23

2.2.1 Definição e Propriedades . . . . . . . . . . . . . . . . . . . 23

2.2 .2 Integral de trajetória . . . . . . . . . . . . . . . . 25

2.2 .3 Limite Semiclássico . . . . . . . . . . . . . . . . . . . 26

2.3 Caso Geral . . . . . . . . . . . . . . . . . . . . . . . . . 28

2.3.1 Estados coerentes e órbitas coadjuntas . . . . . . . . . . 28

2.3.2 Decomposição de Cartan . . . . . . . . . . . . . . 30

2.3.3 Decomposição de Gauss e BCH . . . . . . . . . . . . . . . 31

2.3.4 Os estados coerentes . . . . . . . . . . . . . . . . 33

2.3.5 Supercompleteza . . . . . . . . . . . . . . . 34

2.3.6 Limite clássico . . . . . . . . . . . . . . . . . . 35

$\begin{array}{llr}3 & \text { Estados Coerentes para } S p(4) & \mathbf{3 7}\end{array}$

3.1 O grupo simplético . . . . . . . . . . . . . . . . . . 37

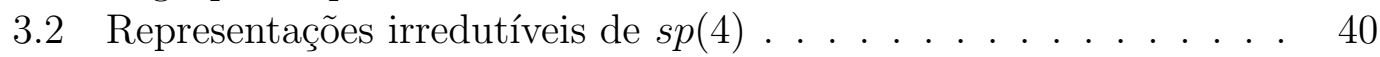

3.3 Escolha de coordenadas . . . . . . . . . . . . . . . . 43

3.4 Estados Coerentes . . . . . . . . . . . . . . . . 45

3.4 .1 Definição . . . . . . . . . . . . . . . . . . 45

3.4.2 Ação dos elementos da álgebra nos estados coerentes . . . 45

3.4 .3 Normalização . . . . . . . . . . . . . . . . . . . . . . 47

3.5 Representações simétricas . . . . . . . . . . . . . . . . . . . . . . 49

3.5.1 Resolução da identidade . . . . . . . . . . . . . . . . . 49

3.5.2 Parêntese de Poisson e coordenadas canônicas . . . . . . . 51

3.6 Caos quântico . . . . . . . . . . . . . . . . . . . . . 53

3.6.1 Distribuição de espaçamento de níveis para sistemas classicamente caóticos . . . . . . . . . . . . . 53

3.6.2 Distribuições de espaçamento de níveis para sistemas classicamente mistos . . . . . . . . . . . . . 56

3.7 Caos quântico em uma Hamiltoniana de $s p(4) \ldots \ldots$. . . . . . . 58 
3.8 O subgrupo maximal $S p(2) \times S p(2) \ldots \ldots \ldots 63$

4 Estados Coerentes de Gazeau-Klauder $\quad 68$

4.1 Introdução . . . . . . . . . . . . . . . . . . . . . . . 69

4.2 Estados coerentes de Gazeau-Klauder . . . . . . . . . . . . . . 70

4.2.1 Exemplos . . . . . . . . . . . . . . . . . 72

4.2.2 Símbolos e desigualdades de Berezin-Lieb . . . . . . . . . 75

4.2 .3 Generalização . . . . . . . . . . . . . . . . . . 77

4.3 Aplicação a magnetismo bidimensional . . . . . . . . . . . . . . . 81

4.3.1 Simetria do oscilador harmônico . . . . . . . . . . . . 83

4.3 .2 Simetria $s u(2) \ldots \ldots \ldots \ldots$. . . . . . . . . . . . 84

$4.3 .3 \quad$ Simetria $s u(1,1) \ldots \ldots \ldots \ldots$. . . . . . . . . . . . 87

4.4 Poço de mínimos duplos . . . . . . . . . . . . . . . . . . . . . 91

4.4.1 O potencial de mínimos duplos clássico . . . . . . . . . . . 91

4.4.2 O potencial de mínimos duplos quântico . . . . . . . . . . 94

4.4.3 Estados coerentes generalizados . . . . . . . . . . 96

$\begin{array}{lll}5 & \text { Conclusões } & 107\end{array}$

A Funções de Wigner e de Husimi $\quad 110$

A.1 Definição e propriedades . . . . . . . . . . . . . . . . . 110

A.2 Exemplo: Oscilador Harmônico . . . . . . . . . . . . . . . . . 113

A.3 O potencial de mínimos duplos . . . . . . . . . . . . . . . 114 


\section{Lista de Figuras}

3.1 Distribuição de espaçamento de níveis para uma representação não-simétrica (esquerda) e para uma simétrica (direita). A linha cheia é a distribuição de Wigner, suposta válida para sistemas classicamente caóticos. . . . . . . . .

3.2 Na coluna da esquerda vemos a distribuição de espaçamento de níveis para diferentes valores de $\epsilon$. A linha cheia é em (a) e (c) é a distribuição de BerryRobnik-Brody, e em (e) é a distribuição de Wigner. Na coluna direita vemos as correspondentes seções de Poincaré. Acompanhamos assim a transição da integrabilidade para o caos nos níveis quântico e clássico. . . . . . . . . .

3.3 A função $W(s)$, definida no texto. Da esquerda para a direita, temos $\epsilon=1.1$, 1.9 e 2.7. A linha cheia são dados numéricos, a linha pontilhada é derivada da distribuição de Berry-Robnik-Brody, e a linha pontilhada vem da distribuição de Wigner. . . . . . . . . . . . . . . . . . .

4.1 Representação pictórica do espaço de estados $\left|n_{1}, n_{2}\right\rangle$. (a) Representação irredutíveis do oscilador harmônico. (b) Representações irredutíveis de $s u(2)$. (c) A série discreta de $s u(1,1)$; linhas acima (abaixo) da linha pontilhada pertencem ao primeiro (segundo) setor definido no texto . . . . . . . . . .

4.2 Níveis de energia e estados estacionários para (a) o poço quártico e (b) o poço

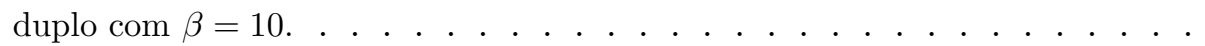

4.3 Densidade de probabilidade $|\langle x \mid J, \gamma\rangle|^{2}$ para estados GK em (a) $t=0$ e (b) $t=T / 4$ onde $T$ é o período clássico. . . . . . . . . . .

4.4 O parâmetro de Mandel pode ser negativo, zero ou positivo, dependendo de $\beta$ e $J$. Em (b) vemos apenas a região de $J$ pequeno. . . . . . . . . . . . . . 97

4.5 A dispersão $\Delta \hat{X} \Delta \hat{P}$ (em unidades de $\hbar$ ) para $J=1$ como função do tempo. Os estados GK não são de mínima incerteza. . . . . . . . . . . . . . .

4.6 As funções de Wigner para estados GK com $J=1$ e $\beta=0$ (acima) e $\beta=10$ (abaixo). Ambas são negativas em regiões do espaço de fase, indicando o caráter não-clássico do estado. . . . . . . . . . . . . . . . . .

4.7 A densidade de probabilidade para o estado GK com $J=500$, em diferentes frações do período clássico. Vemos a superposição coerente de dois estados de mínima incerteza. . . . . . . . . . . . . . . . . . . . . . . 101

4.8 A função de autocorrelação para $\beta=10$ e diferentes valores (pequenos) de $J .103$

4.9 A função de autocorrelação para valores maiores de $J$. . . . . . . . . . . . 104

4.10 Número de estados abaixo da barreira como função de $\beta$. . . . . . . . . 105 
4.11 Picos iniciais da função de autocorrelação. Símbolos diferentes denotam intensidades diferentes, e a linha cheia é o período clássico $T_{c l}$. Note a concordância perfeita entre o primeiro pico e $T_{c l}$ quando $J$ está longe da barreira. . . . . 106

A.1 FWs para $\beta=0$. (a) e (b) mostram curvas de nível e um corte através do plano $p=0$ da FW do estado fundamental. Em (c) e (e) as curvas tracejadas representam as curvas ao longo das quais a FW é um máximo local e a curva cheia é a trajetória clássica. (d) e (f) também são cortes através do plano $p=0$, e as setas indicam os pontos de retorno. . . . . . . . . . . . 116

A.2 FHs para $\beta=0$, em completa analogia com a figura anterior. . . . . . . . 118

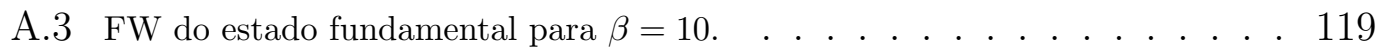

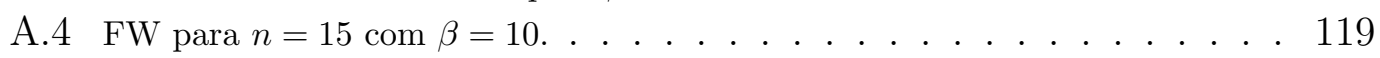

A.5 FHs para $\beta=10$. (a) e (b) estão em analogia com a figura A.1. (c) é um corte através do plano $p=0$ e (d) é um corte através do plano $x=1.58$. Ambas correspondem a $n=3$. . . . . . . . . . . . . . . . . . . . 120

A.6 FHs para $\beta=10$. (a) e (b) são cortes através do plano $p=0$, para $n=2 \mathrm{e}$ $n=4$ respectivamente. (c) e (d) são análogos à figura A.2(e) e (f). . . . . . 121

A.7 FHs de $n=4$ para $\beta=10 \ldots \ldots \ldots$ 


\section{Resumo}

O objetivo desta Tese foi a aplicação da teoria dos estados coerentes para sistemas quânticos não-triviais. A partir da definição de estados coerentes para grupos de Lie compactos em geral, nos dedicamos a uma investigação detalhada da construção de tais estados e de suas propriedades no caso do grupo simplético unitário $S p(4)$, que é extremamente importante tanto em mecânica quântica quanto em mecânica clássica. Esse grupo possui uma complexidade intermediária, que permite um tratamento analítico ainda que apresente propriedades não-triviais do ponto de vista de teoria de representação de álgebras de Lie. Os estados coerentes obtidos nos permitiram uma investigação do limite clássico para sistemas com simetria $S p(4)$ e uma conexão com a teoria do caos em mecânica quântica. Além disso, tratamos uma proposta recente de generalização do conceito de estados coerentes para sistemas de espectro discreto não-degenerado, os estados de Gazeau-Klauder. Esses estados foram aplicados a um probelma de magnetização bidimensional e também ao potencial unidimensional de mínimos duplos, onde observamos o aparecimento dos estados chamados "Gatos de Schrödinger", que consistem na superposição de dois estados de mínima incerteza. 


\section{Abstract}

The subject of the Thesis was the aplication of the coherent states theory to non-trivial quantum systems. Starting from the general definition of coherent states for compact Lie groups, we made a detailed investigation of the construction of these states and its properties in the case of the unitary symplectic group $S p(4)$, which is extremely important in both quantum and classical mechanics. This group has an intermediate complexity, allowing an analytic treatment while presenting non-trivial properties from the point of view of represention theory of Lie algebras. The coherent states so obtained allowed us an investigation of the classical limit of systems with $S p(4)$ symmetry and a conection with the theory of chaos in quantum mechanics. Besides that, we have treated a recent generalization of the concept of coherent states for systems with discrete and nondegenerate spectrum, the Gazeau-Klauder states. These states were applied to a twodimensional magnetization problem and also to the onedimensional double-well potential, where we have observed the appearence of the so-called "Schrödinger cats", which consist in the superposition of two minimum-uncertainty states. 


\section{Capítulo 1}

\section{Introdução}

Estados coerentes foram primeiro definidos para o oscilador harmônico como estados quânticos de mínima incerteza com comportamento quase-clássico, e posteriormente redescobertos em conexão com propriedades de coerência do campo de radiação quantizado [1]. Esses estados coerentes canônicos são hoje observáveis em laboratório e intensamente utilizados em experimentos de ótica quântica [2]. Por outro lado, estados de momentum angular com propriedades análogas ("estados coerentes de spin") apareceram no início dos anos 70, na área de física atômica $[3,4]$. O estado coerente de uma amostra de $N$ átomos de 2 níveis também pode ser gerado em laboratório através da interação com um laser ressonante [5].

Também no início da década de 70 uma generalização para outros grupos de Lie (além daqueles envolvidos no oscilador harmônico e no momentum angular) foi apresentada por Perelomov [6]. Nessa abordagem, mais algébrica, os estados coerentes são vistos como pontos na órbita do estado fundamental do sistema sob ação de um certo grupo de simetria. Essa generalização permitiu que se investigasse em detalhe os estados coerentes de uma classe maior de sistemas, uma vez que requer apenas a presença de uma simetria de Lie.

Os estados coerentes se provaram ferramentas extremamente úteis na obtenção do limite clássico (isto é, funções reais associadas aos observáveis e um parêntese 
de Poisson ligado ao comutador) de sistemas quânticos [7, 8]. O limite clássico de sistemas envolvendo spins, por exemplo, foi obtido por Lieb em 1972 [9], através do mesmo método de desigualdades que empregaremos no Capítulo 4. Do ponto de vista de generalizações, grupos unitários $U(N)$ atraíram a maior parte da atenção [10], devido à sua utilidade na descrição de átomos de $N$ níveis e também porque todas as suas representações irredutíveis são bem conhecidas (o caso do grupo $S U(3)$, por exemplo, foi explorado em detalhe em [11]).

Além (ou talvez aquém) do limite clássico, a descrição da mecânica quântica em uma linguagem própria da mecânica clássica (espaço de fase, equações de Hamilton) possui uma longa história. Os resultados mais importantes nessa direção foram sem dúvida as celebradas funções de Wigner [12] e de Husimi [13], que fornecem representações "clássicas" da matriz densidade [14]. Também nesse contexto os estados coerentes possuem um papel destacado, não só como estados de mínima incerteza mas como ferramenta indispensável na definição do espaço de fase. Recentemente foi sugerida uma generalização das funções de quaseprobabilidade para grupos de Lie arbitrários, na qual os estados coerentes são protagonistas [15].

Outra área da física em que o estados coerentes têm papel relevante é na definição de integrais de trajetória [16]. Os últimos anos têm testemunhado avanços na compreensão de propagadores semiclássicos [17] e problemas de quantização, justamente graças a esse formalismo. A definição de uma integral de trajetória para graus de liberdade de spin, por exemplo, é possível com a utilização de estados coerentes [18]. Uma teoria semiclássica da interação spin-órbita, que foi apresentada recentemente em [19], é inteiramente baseada em estados coerentes .

Tendo em vista a importância crescente dessa área, tivemos neste trabalho dois objetivos principais. Primeiro, estudar em detalhe os estados coerentes de um grupo de Lie não trivial. Escolhemos o grupo simplético unitário $S p(4)$, que possui rank 2 e dimensão (real) 10. Esse grupo é complexo o suficiente para que 
propriedades interessantes estejam presentes, ainda que seja simples o bastante para permitir um tratamento analítico exaustivo. No capítulo 3 definimos os estados coerentes de $S p(4)$ e investigamos todas as suas propriedades, para a seguir estabelecer o limite clássico de sistemas com simetria simplética. Esses resultados estão em discussão no J. Phys. A: Math. Gen., sob o título "Coherent states for the unitary symplectic group", M. Novaes e J.E.M. Hornos.

O grupo simplético é central tanto na mecânica clássica quanto na mecânica quântica como grupo de simetria das equações canônicas. Além disso, tem diversas aplicações em áreas tão diversas quanto ótica quântica [20] e o estudo da evolução do código genético [21]. Acreditamos que nossos resultados encontrarão aplicação nessas áreas, e esperamos que este trabalho possibilite o desenvolvimento de novos projetos envolvendo álgebras de Lie em sistemas físicos de interesse.

Nesse sentido, vale mencionar que nosso grupo de pesquisa possui tradição no estudo dos grupos simpléticos, tendo inclusive desenvolvido fórmulas analíticas para os elementos de matriz da álgebra $s p(4)$, conforme apresentado no Capítulo 3. Esses resultados, assim como muitos outros envolvendo teoria de representação de álgebras de Lie, foram implementados no pacote de rotinas para Maple chamado Killing. Também estão implementados no Killing os harmônicos esféricos $q$-deformados, desenvolvidos por nós no âmbito da álgebra $s u_{q}(2)$ e apresentados em J. Phys. A: Math. Gen. 36, 6733 (Harmonic functions for $s u_{q}(2)$ for $q \in \mathbb{R}$ and $q \rightarrow S^{1}$, M. Novaes, J.E.M. Hornos e E.S. Bernardes).

Investigamos também a integrabilidade de um Hamiltoniana perturbativa construída a partir dos geradores da álgebra $s p(4)$, tanto no nível quântico quanto no nível clássico. Vimos que em geral a distribuição de espaçamento de níveis do sistema é bem descrita pela expressão de Berry-Robnik-Brody [22, 23], que está ligada a sistemas quase-integráveis, de espaço de fase misto. Por outro lado, estudamos a dinâmica do sistema no limite clássico usando os estados coerentes 
desenvolvidos anteriormente, e observamos assim a transição da integrabilidade para o caos. Essa etapa está concluída, e deverá ser divulgado sob o título "Quantum nonintegrability and the classical limit in $s p(4)$ systems", M. Novaes e J.E.M. Hornos.

Finalmente, obtivemos expressões analíticas para os elementos de matriz do grupo simplético $S p(4)$ na representação fundamental, usando a aplicação exponencial. É importante notar que fórmulas explícitas para elementos de matriz de grupos de Lie são raras na literatura. Esses resultados, que estão sendo finalizados, nos permitem uma descrição exaustiva dos subgrupos e dos espaços quociente do grupo $S p(4)$.

Nosso segundo objetivo foi investigar uma generalização recente do conceito de estado coerente proposta por Jean-Pierre Gazeau e John R. Klauder [24]. Esses estados de Gazeau-Klauder são definidos com base apenas nos níveis de energia e não dependem de nenhuma formulação em termos de grupos de Lie. Aplicações recentes desses estados envolvem o átomo de hidrogênio [25], o potencial de Poschl-Teller [26] e o potencial de Morse [27].

Nosso contato com esses estados se deu quando de nossa participação no $23^{\text {rd }}$ International Colloquium on Group Theoretical Methods in Physics, onde apresentamos nossos resultados acerca do grupo $S U(3)$ (Classical and algebraic Hamiltonians with su(3) symmetry, M. Novaes e J.E.M. Hornos, Phys. Atomic. Nuclei 65, 1083). Nesse congresso conhecemos o Prof. Jean-Pierre Gazeau, e iniciamos uma colaboração que resultou na visita de 6 meses feita pelo estudante à Universidade Paris 7.

Durante a visita a Paris, tratamos de estender o formalismo de Gazeau e Klauder a sistemas com mais de um grau de liberdade. Como ilustração, abordamos o problema do potencial termodinâmico de um gás de elétrons em duas dimensões confinado por um potencial harmônico e sujeito a um campo magnético. Esse trabalho foi apresentado à comunidade em J. Phys. A: Math. Gen. 36, 199 
(Multidimensional generalized coherent states, M. Novaes e J.P. Gazeau).

Após o retorno a São Carlos, nos dedicamos a analisar o comportamento desses estados em um potencial unidimensional de mínimos duplos. Essa etapa requereu simulações numéricas extensas, já que esse potencial não possui soluções exatas. Contamos então com a colaboração do Prof. Marcus A.M. de Aguiar, da Unicamp, que já possuía a implementação numérica da solução desse potencial, que fornece os níveis de energia e as funções de onda com dupla precisão.

Dentre todas as propriedades físicas dos estados de Gazeau-Klauder para esse oscilador anarmônico — descritas em detalhe em "Generalized coherent states for the double-well potential", M. Novaes, M.A.M. de Aguiar e J.E.M. Hornos, J. Phys. A: Math. Gen. 36, 5773 - destacamos o regime de altas energias. Nesse regime observamos interessantes bifurcações na evolução da função de autocorrelação, e também o surgimento de um "Gato de Schrödinger": uma superposição coerente de duas gaussianas de mínima incerteza.

Ainda no contexto do potencial de mínimos duplos, estudamos as funções de Wigner e de Husimi para os estados estacionários. Observamos propriedades de localização interessantes, especialmente no caso da função de Husimi, que se concentra sobre a trajetória clássica mesmo para estados abaixo da barreira. Essas propriedades foram discutidas em J. Opt. B: Quantum Semiclass. Opt. 5, S342 (Wigner and Husimi functions in the double-well potential, M. Novaes).

Esta Tese está organizada como se segue: no Capítulo 2 apresentamos em detalhe os estados coerentes do oscilador harmônico e do momentum angular. Tratamos também da generalização para grupos de Lie compactos. No Capítulo 3 aplicamos o formalismo ao grupo simplético unitário $S p(4)$, obtendo os estados coerentes associados a diferentes representações irredutíveis. Investigamos também a integrabilidade clássica e quântica de uma Hamiltoniana perturbativa. No Capítulo 4 nos voltamos para os estados de Gazeau-Klauder. Concluímos no Capítulo 5. O Apêndice A trata das funções de Wigner e de Husimi. 


\section{Capítulo 2}

\section{Estados Coerentes}

Neste capítulo será introduzido o conceito de estados coerentes com base nos exemplos tradicionais: oscilador harmônico e momentum angular. A partir desses casos simples vamos passar ao caso mais geral de grupos de Lie compactos. Veremos as propriedades típicas desses estados e sua conexão com o limite clássico da mecânica quântica. Parte do material apresentado neste capítulo já apareceu em [28].

\subsection{Oscilador Harmônico}

\subsubsection{Definição e Propriedades}

Os estados coerentes do oscilador harmônico, chamados canônicos, foram descobertos por Schrödinger, que notou suas propriedades semiclássicas. Esses estados permaneceram uma curiosidade até o começo da década de 60 (em parte porque não foi possível encontrar estados análogos para o átomo de hidrogênio [29]), quando Glauber [30] estabeleceu as relações entre esses estados e propriedades do campo de radiação. Os estudos de Klauder envolvendo famílias supercompletas de estados [16] e de Sudarshan em ótica quântica [31] também ajudaram a po-

pularizar esses estados, que hoje são construídos e manipulados em laboratório, 
usando cavidades [2], e são centrais no estudo de fenômenos como decoerência e teletransporte [32]. Como nosso interesse é a generalização do conceito de estado coerente, adotaremos aqui uma abordagem algébrica.

A álgebra de Lie associada ao oscilador harmônico, chamada álgebra de WeylHeisenberg,

$$
\left[a, a^{\dagger}\right]=1, \quad\left[\hat{n}, a^{\dagger}\right]=a^{\dagger}
$$

é gerada pelos operadores $\left\{a, a^{\dagger}, \hat{n}, 1\right\}$ definidos por

$$
a^{\dagger}=\sqrt{\frac{m \omega}{\hbar}} X+i \frac{1}{\sqrt{m \omega \hbar}} P, \quad \hat{n}=a^{\dagger} a
$$

onde $X$ e $P$ representam a posição e o momentum de uma partícula que se move em uma dimensão.

As relações de comutação acima são compatíveis com a relação de incerteza $\Delta X \Delta P \geq \frac{\hbar}{2}$, que implica que a partícula não possui valores bem definidos de posição e de momentum simultaneamente $\left(\Delta f=\sqrt{\left\langle(f-\langle f\rangle)^{2}\right\rangle}\right.$ representa o desvio quadrático médio da variável $f$ ). Sendo assim, não é possível representar o sistema como um "ponto" em um espaço de fase, como é tradicionalmente feito na mecânica clássica.

Essa álgebra pode ser representada por matrizes de dimensão infinita que satisfazem $(n=0,1,2, \ldots)$

$$
\hat{n}|n\rangle=n|n\rangle, \quad a|n\rangle=\sqrt{n}|n-1\rangle, \quad a^{\dagger}|n\rangle=\sqrt{n+1}|n+1\rangle,
$$

e a Hamiltoniana do oscilador harmônico (medida a partir da energia de ponto zero) é proporcional ao operador de número

$$
H=\frac{m}{2}\left(\omega^{2} X^{2}+P^{2}\right)=\hbar \omega\left(\hat{n}+\frac{1}{2}\right) .
$$


O estado coerente associado a essa álgebra (também chamado estado coerente canônico) é definido como:

$$
|\alpha\rangle=e^{\alpha a^{\dagger}-\alpha^{*} a}|0\rangle=\mathrm{e}^{-|\alpha|^{2} / 2} \mathrm{e}^{\alpha a^{\dagger}}|0\rangle=\mathrm{e}^{-|\alpha|^{2} / 2} \sum_{n=0}^{\infty} \frac{\alpha^{n}}{\sqrt{n !}}|n\rangle, \quad \alpha \in C .
$$

Vemos que a cada ponto do plano complexo corresponde um estado coerente. A seguir as principais propriedades desses estados:

$$
\begin{aligned}
\text { Autoestados } & : \quad a|\alpha\rangle=\alpha|\alpha\rangle, \\
\text { Estabilidade } & : \quad e^{-i H t / \hbar}|\alpha\rangle=e^{-i \omega t / 2}\left|\alpha e^{i \omega t}\right\rangle, \\
\text { Mínima incerteza } & : \Delta X \Delta P=\frac{\hbar}{2}, \\
\text { Não-ortogonalidade } & :\langle\alpha \mid \beta\rangle=\exp \left\{-\frac{1}{2}|\alpha|^{2}+\beta \alpha^{*}-\frac{1}{2}|\beta|^{2}\right\} \\
\text { Resolução da identidade } & : \quad \frac{1}{\pi} \int d^{2} \alpha|\alpha\rangle\langle\alpha|=1, \\
\text { Variável de ação } & :\langle\alpha|H| \alpha\rangle=\hbar \omega\left(|\alpha|^{2}+\frac{1}{2}\right) .
\end{aligned}
$$

A integral em (2.10) é feita sobre todo o plano complexo. Uma família de estados é dita supercompleta se possuir um subconjunto completo, e uma tal família não pode possuir todos seus elementos ortogonais entre si. Como veremos, a não ortogonalidade é uma característica geral dos estados coerentes, assim como a supercompleteza.

Cada uma das propriedades listadas acima foi ponto de partida para uma generalização diferente dos estados coerentes. A equação de autovalores (2.6) não pode ser satisfeita para álgebras compactas, mas funciona para $s u(1,1)$, por exemplo [33]. A estabilidade é uma propriedade bastante desejável: o resultado da evolução de um estado coerente inicial $\left|\alpha_{0}\right\rangle$ é um outro estado coerente $\left|\alpha_{0} e^{i t}\right\rangle$, que no plano complexo corresponde a uma rotação do estado inicial. Estados quânticos de mínima incerteza, por outro lado, foram estudados em uma série 
de trabalhos por Nieto e colaboradores [34]. A supercompleteza foi a principal propriedade exigida por Klauder em seu trabalho seminal [16], e segue sendo reconhecida como central, ainda que famílias de estados coerentes sem resolução da identidade possam ser definidas [35].

A fim de obtermos o limite clássico, discutiremos brevemente integrais de trajetória na seção a seguir. Além disso, nossa apresentação contém os elementos necessários a uma discussão preliminar do limite clássico. Um tratamento recente e extremamente detalhado de integrais de trajetória usando estados coerentes canônicos pode ser encontrada em [17].

\subsubsection{Integral de trajetória}

Dados dois estados coerentes $\left|\alpha_{0}\right\rangle$ e $\left|\alpha_{N}\right\rangle$, podemos perguntar qual a probabilidade de que uma partícula inicialmente em $\left|\alpha_{0}\right\rangle$ esteja em $\left|\alpha_{N}\right\rangle$ depois de um tempo $t$. Ora, a resposta é dada pelo propagador [36]:

$$
K\left(\alpha_{0}, \alpha_{N}, t\right)=\left\langle\alpha_{N}\left|e^{-\frac{i}{\hbar} H t}\right| \alpha_{0}\right\rangle
$$

Para calcular essa grandeza, dividimos o intervalo de tempo em $N$ intervalos pequenos de tamanho $\varepsilon=t / N$ e usamos $N-1$ vezes a crucial propriedade $\pi^{-1} \int d^{2} \alpha|\alpha\rangle\langle\alpha|=1$ para obter

$$
K\left(\alpha_{0}, \alpha_{N}, t\right)=\lim _{N \rightarrow \infty} \int \cdots \int \prod_{n=1}^{N-1} \frac{d^{2} \alpha_{n}}{\pi} \prod_{k=1}^{N}\left\langle\alpha_{k}\left|e^{-\frac{i}{\hbar} \varepsilon H}\right| \alpha_{k-1}\right\rangle .
$$

Considerando $\varepsilon \ll 1$, fazemos a aproximação

$$
\left\langle\alpha_{k}\left|e^{-\frac{i}{\hbar} \varepsilon H}\right| \alpha_{k-1}\right\rangle \simeq\left\langle\alpha_{k}\left|1-\frac{i}{\hbar} \varepsilon H\right| \alpha_{k-1}\right\rangle=\left\langle\alpha_{k} \mid \alpha_{k-1}\right\rangle\left(1-\frac{i}{\hbar} \varepsilon \frac{\left\langle\alpha_{k}|H| \alpha_{k-1}\right\rangle}{\left\langle\alpha_{k} \mid \alpha_{k-1}\right\rangle}\right) .
$$


Sabemos que

$$
\left\langle\alpha_{k} \mid \alpha_{k-1}\right\rangle=\exp \left\{-\frac{1}{2}\left|\alpha_{k}\right|^{2}+\alpha_{k-1} \alpha_{k}^{*}-\frac{1}{2}\left|\alpha_{k-1}\right|^{2}\right\}
$$

e nessa expressão somamos e subtraímos os termos $\left|\alpha_{k-1}\right|^{2}, \alpha_{k} \alpha_{k-1}^{*} / 2$ e $\left|\alpha_{k-1}\right|^{2} / 2$. Após rearranjar os fatores adequadamente, temos

$$
\left\langle\alpha_{k} \mid \alpha_{k-1}\right\rangle=\exp \left\{-\frac{1}{2} \alpha_{k} \Delta_{k}^{*}-\frac{1}{2} \alpha_{k-1}^{*} \Delta_{k}+\alpha_{k-1} \Delta_{k}^{*}\right\}
$$

onde $\Delta_{k}=\alpha_{k}-\alpha_{k-1}$. Considerando que no limite $\varepsilon \rightarrow 0$ temos

$$
\frac{\left\langle\alpha_{k}|H| \alpha_{k-1}\right\rangle}{\left\langle\alpha_{k} \mid \alpha_{k-1}\right\rangle} \rightarrow \mathcal{H}\left(\alpha_{k}, \alpha_{k}^{*}\right)
$$

fazemos

$$
\left\langle\alpha_{k}\left|e^{-\frac{i}{\hbar} \varepsilon H}\right| \alpha_{k-1}\right\rangle \simeq\left\langle\alpha_{k} \mid \alpha_{k-1}\right\rangle e^{-\frac{i}{\hbar} \varepsilon \mathcal{H}\left(\alpha_{k}, \alpha_{k}^{*}\right)}
$$

O propagador em (2.13) fica portanto

$$
\begin{gathered}
K\left(\alpha_{0}, \alpha_{N}, t\right)=\lim _{\varepsilon \rightarrow 0} \int \ldots \int \prod_{n=1}^{N-1} \frac{d^{2} \alpha_{n}}{\pi} \prod_{k=1}^{N} e^{-\frac{i}{\hbar} S_{k}}=\lim _{\varepsilon \rightarrow 0} \int \cdots \int \prod_{n=1}^{N-1} \frac{d^{2} \alpha_{n}}{\pi} e^{-i S / \hbar} \\
S=\sum_{k=1}^{N} i \hbar\left(\frac{1}{2} \alpha_{k} \Delta_{k}^{*}+\frac{1}{2} \alpha_{k-1}^{*} \Delta_{k}-\alpha_{k-1} \Delta_{k}^{*}\right)-\varepsilon \mathcal{H}\left(\alpha_{k}, \alpha_{k}^{*}\right) .
\end{gathered}
$$

Podemos tomar agora o limite para o contínuo, no qual

$$
S=\int_{0}^{t}\left[i \hbar \frac{\alpha^{*} \dot{\alpha}-\alpha \dot{\alpha}^{*}}{2}-\mathcal{H}\left(\alpha, \alpha^{*}\right)\right] d t
$$

e teremos portanto uma Lagrangeana

$$
\mathcal{L}\left(\alpha, \alpha^{*}\right)=i \hbar \frac{\alpha^{*} \dot{\alpha}-\alpha \dot{\alpha}^{*}}{2}-\mathcal{H}\left(\alpha, \alpha^{*}\right)
$$


Se notarmos que

$$
\left\langle\alpha\left|\frac{\partial}{\partial \alpha}\right| \alpha\right\rangle=\frac{\alpha^{*}}{2}
$$

e

$$
\frac{\partial}{\partial t}=\dot{\alpha} \frac{\partial}{\partial \alpha}+\dot{\alpha}^{*} \frac{\partial}{\partial \alpha^{*}}
$$

podemos escrever

$$
\mathcal{L}\left(\alpha, \alpha^{*}\right)=\left\langle\alpha\left|i \hbar \frac{\partial}{\partial t}-H\right| \alpha\right\rangle
$$

\subsubsection{Limite Semiclássico}

Entendemos como limite semiclássico a situação em que a ação de qualquer trajetória é muito maior que a constante de Planck

$$
S \gg \hbar
$$

Nesse caso vale o princípio de mínima ação $\delta S=0$, pois empregamos a aproximação de fase estacionária ao propagador. A equação de Lagrange aplicada a (2.22) fornece as equações de Hamilton dadas por

$$
\dot{\alpha}=\frac{1}{i \hbar} \frac{\partial \mathcal{H}}{\partial \alpha^{*}}
$$

e sua conjugada. Podemos escrevê-las em termos de um parêntese de Poisson:

$$
\{f, g\}=\frac{1}{i \hbar}\left\{\frac{\partial f}{\partial \alpha} \frac{\partial g}{\partial \alpha^{*}}-\frac{\partial f}{\partial \alpha^{*}} \frac{\partial g}{\partial \alpha}\right\}
$$

e podemos reduzir este parêntese a uma expressão mais familiar definindo $\alpha=$ $(q+i p) / \sqrt{2 \hbar}$. Usando essas novas variáveis teremos

$$
\mathcal{L}(q, p)=\frac{p \dot{q}-q \dot{p}}{2}-\mathcal{H}(q, p)
$$


e

$$
\{f, g\}=\left\{\frac{\partial f}{\partial q} \frac{\partial g}{\partial p}-\frac{\partial f}{\partial p} \frac{\partial g}{\partial q}\right\}
$$

As equações de movimento (2.27) tornam-se as equações de Hamilton:

$$
\dot{q}=\frac{\partial \mathcal{H}}{\partial p}, \quad \dot{p}=-\frac{\partial \mathcal{H}}{\partial q},
$$

e portanto o plano complexo é identificado naturalmente com o espaço de fase de um sistema unidimensional. No limite semi-clássico os estados coerentes podem ser vistos como pontos que se movem nesse plano sobre as trajetórias geradas pela Hamiltoniana clássica $\mathcal{H}(q, p)$.

Consideramos que as variáveis $\{q, p\}$ descrevem o movimento de uma partícula quântica qualquer. Entretanto, devemos também levar em conta o grau de liberdade intrínseco chamado spin. O spin é algebricamente equivalente ao momentum angular, e é disso que trataremos na seção seguinte.

\subsection{Momentum Angular}

\subsubsection{Definição e Propriedades}

Em mecânica quântica, a cada componente do momentum angular de uma partícula associa-se um operador hermitiano, $J_{x}, J_{y}$ ou $J_{z}$. Com base nesses operadores, que obedecem a relação de comutação $\left[J_{i}, J_{j}\right]=i J_{k}(i, j, k$ cíclicos $)$, definimos $J_{ \pm}=J_{x} \pm i J_{y}$ de forma a obtermos as relações de comutação abaixo:

$$
\left[J_{+}, J_{-}\right]=2 J_{z}, \quad\left[J_{z}, J_{ \pm}\right]= \pm J_{ \pm}
$$


Essa álgebra de Lie é denotada $s u(2)$, e suas representações unitárias irredutíveis são etiquetadas por um número $j$ tal que $2 j \in \mathbb{N}$ e dadas por

$$
\begin{aligned}
J_{z}|j, m\rangle & =m|j, m\rangle, \\
J_{ \pm}|j, m\rangle & =\sqrt{(j \mp m)(j \pm m+1)}|j, m \pm 1\rangle .
\end{aligned}
$$

Essas relações de comutação levam à relação de incerteza $\Delta J_{x} \Delta J_{y} \geq \frac{\hbar}{2}\left|\left\langle J_{z}\right\rangle\right|$. O estado coerente, analogamente a $(2.5)$, é definido por $(z \in \mathbb{C})$

$|z\rangle=e^{z J_{+}-z^{*} J_{-}}|j,-j\rangle=\frac{e^{z J_{+}}}{\left(1+|z|^{2}\right)^{j}}|j,-j\rangle=\sum_{m=-j}^{j}\left(\begin{array}{c}2 j \\ j+m\end{array}\right)^{\frac{1}{2}} \frac{z^{j+m}}{\left(1+|z|^{2}\right)^{j}}|j, m\rangle$,

e possui as seguintes propriedades:

$$
\begin{array}{rll}
\text { Não-ortogonalidade } & : & \left\langle z_{1} \mid z_{2}\right\rangle=\frac{\left(1+z_{1}^{*} z_{2}\right)^{2 j}}{\left(1+\left|z_{1}\right|^{2}\right)^{j}\left(1+\left|z_{2}\right|^{2}\right)^{j}}, \\
\text { Valores Médios } & : \quad\left\langle z\left|J_{z}\right| z\right\rangle=j \frac{|z|^{2}-1}{|z|^{2}+1},\left\langle z\left|J_{+}\right| z\right\rangle=\frac{2 j z^{*}}{1+|z|^{2}}(2.37) \\
\text { Estabilidade } & : \quad e^{a J_{z}}|z\rangle=e^{-j a}\left|z e^{a}\right\rangle, \\
\text { Mínima incerteza } & : \quad \Delta J_{x} \Delta J_{y}=\frac{\hbar}{2}\left|\left\langle J_{z}\right\rangle\right|, \\
\text { Resolução da identidade } & : \quad \int d \mu\left(z, z^{*}\right)|z\rangle\langle z|=1,
\end{array}
$$

onde

$$
d \mu\left(z, z^{*}\right)=\frac{2 j+1}{4 \pi} \frac{d z d z^{*}}{\left(1+|z|^{2}\right)^{2}}
$$

Outra maneira de visualizarmos esses estados é através de uma projeção estereográfica do plano sobre a superfície esférica dada por

$$
z=e^{i \varphi} \tan \frac{\theta}{2}
$$


Nesse caso os estados coerentes ficam

$$
|\theta, \varphi\rangle=\sum_{m=-j}^{j}\left(\begin{array}{c}
2 j \\
j+m
\end{array}\right)^{\frac{1}{2}}(\cos \theta)^{j-m}(\sin \theta)^{j+m} e^{i(j+m) \varphi}|j, m\rangle
$$

e a medida de integração fica

$$
d \mu(\theta, \varphi)=\frac{2 j+1}{4 \pi} \sin \theta d \theta d \varphi
$$

Esta representação tem um apelo geométrico forte, uma vez que o grupo $S O(3)$ (usamos letras minúsculas para designar álgebras, e maiúsculas para designar grupos) está ligado a rotações tridimensionais. Essa relação fica ainda mais evidente quando observamos o valor médio dos geradores:

$$
\begin{aligned}
\left\langle\theta, \varphi\left|J_{z}\right| \theta, \varphi\right\rangle & =j \cos \theta, \\
\left\langle\theta, \varphi\left|J_{x}\right| \theta, \varphi\right\rangle & =j \sin \theta \cos \varphi, \\
\left\langle\theta, \varphi\left|J_{y}\right| \theta, \varphi\right\rangle & =j \sin \theta \sin \varphi .
\end{aligned}
$$

Vemos que o estado coerente da álgebra de momento angular se comporta como um vetor clássico de tamanho $j$. Essa superfície esférica é o espaço de fase associado a um sistema de spin $j$. Analogamente ao caso do oscilador harmônico, introduzimos a integral de trajetória.

\subsubsection{Integral de trajetória}

O cálculo do propagador neste caso é análogo àquele apresentado para o oscilador harmônico, exceto pelo fato de que agora a medida $d^{2} \alpha / \pi$ deve ser substituída por $d \mu\left(z, z^{*}\right)$ e que

$$
\left\langle z_{k} \mid z_{k-1}\right\rangle=\frac{\left(1+z_{k}^{*} z_{k-1}\right)^{2 j}}{\left(1+\left|z_{k}\right|^{2}\right)^{j}\left(1+\left|z_{k-1}\right|^{2}\right)^{j}} .
$$


Nesta expressão, somamos e subtraímos $\left|z_{k-1}\right|^{2}$ dentro do numerador de forma que $\left(1+z_{k}^{*} z_{k-1}\right)=\left(1+\left|z_{k-1}\right|^{2}+\Delta_{k}^{*} z_{k-1}\right)$, onde $\Delta_{k}=z_{k}-z_{k-1}$. No denominador, somamos e subtraímos $\left|z_{k-1}\right|^{2}$ e $z_{k} z_{k-1}^{*}$ de forma que $\left(1+\left|z_{k}\right|^{2}\right)=\left(1+\left|z_{k-1}\right|^{2}+\right.$ $\left.\Delta_{k}^{*} z_{k}+\Delta_{k} z_{k-1}^{*}\right)$. Dessa maneira, temos

$$
\left\langle z_{k} \mid z_{k-1}\right\rangle=\frac{\left(1+\left|z_{k-1}\right|^{2}+\Delta_{k}^{*} z_{k-1}\right)^{2 j}}{\left(1+\left|z_{k-1}\right|^{2}+\Delta_{k}^{*} z_{k}+\Delta_{k} z_{k-1}^{*}\right)^{j}\left(1+\left|z_{k-1}\right|^{2}\right)^{j}} .
$$

Supondo $\Delta_{k}$ da ordem de $\varepsilon$ quando $\varepsilon \rightarrow 0$, expandimos a expressão anterior em série de Taylor

$$
\left\langle z_{k} \mid z_{k-1}\right\rangle \simeq 1+j \frac{\Delta_{k}^{*} z_{k-1}-\Delta_{k} z_{k-1}^{*}}{1+\left|z_{k-1}\right|^{2}} \simeq \exp \left\{j \frac{\Delta_{k}^{*} z_{k-1}-\Delta_{k} z_{k-1}^{*}}{1+\left|z_{k-1}\right|^{2}}\right\}
$$

e é fácil ver que neste caso a Lagrangeana será dada por

$$
\mathcal{L}\left(z, z^{*}\right)=i \hbar j \frac{\left(\dot{z} z^{*}-\dot{z}^{*} z\right)}{1+|z|^{2}}-\mathcal{H}\left(z, z^{*}\right)
$$

e que novamente podemos escrevê-la como $\mathcal{L}\left(z, z^{*}\right)=\left\langle z\left|i \hbar \frac{\partial}{\partial t}-H\right| z\right\rangle$.

É importante deixar claro que todo o formalismo desenvolvido nesta seção (e também em 2.1.2) é exclusivamente quântico, ou seja, as integrações são feitas sobre o espaço dos estados coerentes, não sobre um espaço de fase clássico dado a priori, como no formalismo original de Feynman [36]. Uma discussão recente e detalhada a respeito de integrais de trajetória usando estados coerentes de $s u(2)$ pode ser encontrada em [18].

\subsubsection{Limite Semiclássico}

A equação de Lagrange neste caso fornece

$$
\dot{z}=-i \frac{\left(1+|z|^{2}\right)^{2}}{2 j \hbar} \frac{\partial \mathcal{H}}{\partial z^{*}}
$$


e sua conjugada. Podemos escrevê-las novamente em termos de um parêntese de Poisson:

$$
\{f, g\}=\frac{1}{i \hbar} \frac{\left(1+|z|^{2}\right)^{2}}{2 j}\left\{\frac{\partial f}{\partial z} \frac{\partial g}{\partial z^{*}}-\frac{\partial f}{\partial z^{*}} \frac{\partial g}{\partial z}\right\}
$$

ou, usando as variáveis da projeção estereográfica,

$$
\{f, g\}=\frac{1}{\hbar j \sin \theta}\left\{\frac{\partial f}{\partial \varphi} \frac{\partial g}{\partial \theta}-\frac{\partial f}{\partial \theta} \frac{\partial g}{\partial \varphi}\right\}
$$

Novamente podemos reduzir essa expressão a uma forma mais familiar, definindo

$$
\frac{q+i p}{\sqrt{2 \hbar j}}=\frac{z}{\sqrt{1+|z|^{2}}}
$$

ou inversamente

$$
z=\frac{q+i p}{\sqrt{2 \hbar j-q^{2}-p^{2}}} .
$$

Note que a relação

$$
q^{2}+p^{2}=2 \hbar j \frac{|z|^{2}}{1+|z|^{2}}
$$

obtida a partir de (2.53) impõe a desigualdade

$$
0 \leq q^{2}+p^{2} \leq 2 \hbar j
$$

Usando essas novas variáveis temos novamente

$$
\mathcal{L}(q, p)=\frac{p \dot{q}-q \dot{p}}{2}-\mathcal{H}(q, p)
$$

$\mathrm{e}$

$$
\{f, g\}=\left\{\frac{\partial f}{\partial q} \frac{\partial g}{\partial p}-\frac{\partial f}{\partial p} \frac{\partial g}{\partial q}\right\}
$$

É importante frisar que neste caso o espaço de fase não é simplesmente $\mathbb{R}^{2}$ devido à restrição (2.56). O espaço de fase mais natural para este sistema é a superfície esférica. Podemos utilizar o plano complexo se o entendermos como 
advindo de uma projeção estereográfica, mas então a métrica neste plano será não-trivial como vemos em (2.51). Outro aspecto essencial é o fato de que nos resultados finais a constante de Planck $\hbar$ aparece sempre multiplicada por $j$. Isso indica que o limite $\hbar \rightarrow 0$ deve ser acompanhado por $j \rightarrow \infty$ de forma que $\hbar j$ seja mantido constante.

Tendo visto em detalhe a construção dos estados coerentes e do espaço de fase quântico para os casos mais simples, passamos agora a uma análise mais geral.

\subsection{Caso Geral}

Consideraremos como caso geral o cálculo dos estados coerentes para uma representação qualquer de um dado grupo de Lie compacto (o oscilador harmônico não se encaixa nesta restrição mas pode ser considerado um caso limite [4]). O espaço de fase natural para um sistema quântico - que no caso do oscilador harmônico é o plano complexo e no caso do momentum angular é a superfície esférica - é dado pelo quociente de seu grupo de simetria $G$ por um subgrupo $H$. Um tratamento detalhado desse formalismo pode ser encontrado em [6, 37].

Veremos a seguir de forma introdutória a teoria dos espaços quociente e das órbitas coadjuntas. Apresentaremos também a decomposição de Cartan de uma álgebra de Lie e a decomposição de Gauss de um grupo de Lie, que são importantes na definição e na operacionalização dos estados coerentes.

\subsubsection{Estados coerentes e órbitas coadjuntas}

A relação entre estados coerentes e órbitas coadjuntas de grupos de Lie é conhecida há bastante tempo, e a literatura sobre esse assunto (e sua conexão com a teoria de quantização geométrica) é vasta. Nós esboçamos aqui apenas uma descrição breve da teoria e alguns de seus resultados principais, sem entrar em detalhes. Exposições recentes do assunto podem ser encontradas em [11, 37, 38]. 
Seja $G$ um grupo de Lie com uma ação (à esquerda) sobre uma variedade diferenciável $X$. Se essa ação, denotada $g x$ (onde $g \in G$ e $x \in X$ ), é transitiva, o que significa que dados $x, y \in X$ sempre existe $g \in G$ tal que $x=g y$, então $X$ é dito um espaço homogêneo. Todos esses espaços são difeomórfos a espaços quociente $G / H$, onde $H$ é um subgrupo fechado de $G$ (elementos de $G / H$ são da forma $g H$, e $G$ possui uma ação natural sobre esse espaço dada por $\left.g H \mapsto g^{\prime} g H\right)$.

Para vermos isso, consideremos o ponto $x_{0} \in X$ e seja $H_{0}$ seu grupo de estabilidade, dado por $H_{0}=\left\{h \in G \mid h x_{0}=x_{0}\right\}$. Uma vez que $X$ é homogêneo, para qualquer $x \in X$ existe $g \in G$ para o qual $x=g x_{0}$. A identificação $x \mapsto g H_{0}$ é então um difeomorfismo entre $X$ e $G / H_{0}$. De fato, dado um espaço geral $X$, a órbita de $x \in X$ sob ação de $G$ é o conjunto $G x=\{y \in X \mid g \in G\}$ e portanto $X$ consiste em uma coleção de $G$-orbitas disjuntas, cada uma delas sendo um espaço homogêneo.

Por outro lado, $G$ possui uma ação natural, a ação adjunta, sobre sua álgebra $\mathfrak{g}$, na qual $A \mapsto g(A)=g A g^{-1}$ para qualquer $A \in \mathfrak{g}$ (uma vez que estamos interessados apenas em grupos matriciais, o produto $g A$ pode ser considerado como o produto usual de matrizes). Isso induz uma ação de $G$ sobre $\mathfrak{g}^{*}$, o dual de $\mathfrak{g}$, na qual $\rho \mapsto \rho_{g}$. Essa é a chamada ação coadjunta, e $\rho_{g} \in \mathfrak{g}^{*}$ é definido por $\rho_{g}(A)=\rho(g(A)), A \in \mathfrak{g}$.

O conjunto $\mathcal{O}_{\rho}=\left\{\rho_{g} \mid g \in G\right\}$ é chamado a órbita coadjunta de $\rho$ sob ação de $G$. É um espaço homogêneo e portanto difeomórfo ao quociente $G / H_{\rho}$, onde $H_{\rho}=\left\{g \in G \mid \rho_{g}=\rho\right\}$ é o grupo de estabilidade de $\rho$. É claro que, dado $\rho$, sua órbita coadjunta pode ser usada para definir uma representação $\mathcal{R}_{\rho}$ da álgebra $\mathfrak{g}$ como funções contínuas sobre $G / H_{\rho}$, na qual

$$
\mathcal{R}_{\rho}(A)(x)=\rho_{x}(A), \quad A \in \mathfrak{g}, \quad x \in G / H_{\rho}
$$

Essa representação é naturalmente equipada com um parêntese de Poisson $\{\cdot, \cdot\}$ 
induzido pelo produto de Lie da álgebra $[\cdot, \cdot]$ de acordo com $\left\{\mathcal{R}_{\rho}(A), \mathcal{R}_{\rho}(B)\right\}(x)=$ $\rho_{x}([A, B])$. Esse parêntese será escrito em coordenadas locais na seção 2.3.6

Vimos portanto que os espaços quociente $G / H$ podem ser encarados como espaços de fase, e que a álgebra de Lie $\mathfrak{g}$ correspondente a $G$ pode ser representada por funções sobre esse espaço. Façamos agora a conexão com estados coerentes. Seja $\mathbb{H}$ um espaço de Hilbert que carrega uma representação unitária irredutível de $\mathfrak{g}$, denotada $T$. A todo vetor $|0\rangle \in \mathbb{H}$ corresponde um elemento $\rho_{0} \in \mathfrak{g}^{*}$ cuja ação sobre $\mathfrak{g}$ é dada por $\rho_{0}(A)=\langle 0|T(A)| 0\rangle, A \in \mathfrak{g}$. Portanto, existe uma identificação natural entre a órbita coadjunta $\rho_{g}$ e o conjunto de vetores $|g\rangle=T(g)|0\rangle$, na qual

$$
\rho_{g}(A)=\langle g|T(A)| g\rangle=\langle 0|T(g(A))| 0\rangle
$$

O conjunto dos estados coerentes $|g\rangle, g \in G$, é portanto topologicamente equivalente ao quociente $G / H$, onde $H$ é o grupo de isotropia do vetor de referência $|0\rangle$. Esse vetor é em geral tomado como o peso máximo (ou mínimo) da representação, mas estados coerentes construídos a partir de pesos mais gerais possuem muitas das propriedades usuais $[1,6]$. Veremos a seguir teoremas de decompoisição que são indispensáveis em teoria de grupos e na abordagem dos estados coerentes em particular.

\subsubsection{Decomposição de Cartan}

Todas as álgebras de Lie clássicas possuem uma decomposição de Cartan do tipo [39]

$\left[H_{i}, H_{j}\right]=0, \quad\left[H_{i}, E_{\alpha}\right]=\alpha_{i} E_{\alpha}, \quad\left[E_{\alpha}, E_{-\alpha}\right]=\alpha^{i} H_{i}, \quad\left[E_{\alpha}, E_{\beta}\right]=N_{\alpha \beta} E_{\alpha+\beta}$.

Os operadores $H_{i}, i=1 . . r$, compõem a chamada subálgebra (comutativa) de Cartan, e $r$ é chamado o rank da álgebra. Os operadores de levantamento $E_{\alpha}$, 
$\alpha=1 . . k$, são também chamados de raízes positivas da álgebra. Analogamente, os operadores de abaixamento $E_{-\alpha}$ são chamados raízes negativas (note que $r+2 k=$ dimensão da álgebra). As outras quantidades são números complexos. No caso do momentum angular, $J_{z}$ é o único componente da subálgebra de Cartan, enquanto que $J_{+}$e $J_{-}$são os operadores de levantamento e abaixamento.

Vamos denotar por $|0\rangle$ o vetor "mais baixo" (também chamado peso mínimo ou estado fundamental) de uma dada representação unitária de $A$ :

$$
E_{-\alpha}|0\rangle=0, \quad \text { para todo } \alpha
$$

Além de ser aniquilado por todos os operadores de abaixamento, o estado fundamental pode eventualmente sê-lo também por alguns dos de levantamento:

$$
E_{\beta}|0\rangle=0, \quad \text { para alguns } \beta \text {. }
$$

A união de todos os operadores $E_{\beta}$ que satisfazem (2.63) com seus conjugados $E_{-\beta}$ e com a subálgebra de Cartan gera uma álgebra, h. A exponencial dessa álgebra, $H_{0}=e^{\mathfrak{h}}$, é chamado o subgrupo de isotropia do vetor $|0\rangle$, uma vez que $h|0\rangle=e^{i \gamma}|0\rangle$ para todo $h \in H_{0}$.

\subsubsection{Decomposição de Gauss e BCH}

Na decompoisção de Gauss escrevemos uma matriz genérica $g$ pertencente ao grupo como um produto do tipo $P D N$, onde $P$ pertence ao subgrupo gerado por todas as raízes positivas, $D$ pertence ao subgrupo abeliano gerado pela subálgebra de Cartan e $N$ pertence ao subgrupo gerado por todas as raízes negativas:

$$
g=\exp \left\{\sum_{\alpha=1}^{k} \tau_{\alpha}^{+} E_{\alpha}\right\} \exp \left\{\sum_{i=1}^{r} h_{i} H_{i}\right\} \exp \left\{\sum_{\alpha=1}^{k} \tau_{\alpha}^{-} E_{-\alpha}\right\} .
$$


As variáveis $\left(\tau_{\alpha}^{ \pm}, h_{i}\right)$ são determinadas univocamente pelos elementos de matriz de $g$. Esse teorema é uma forma de "abrir" a exponencial da soma de vários elementos em um produto de exponenciais envolvendo a soma de menos elementos.

É possível abrir ainda mais essa expressão usando as chamadas "fórmulasBCH". Se $A$ é uma álgebra de Lie e $x, y \in A$ dois de seus elementos, sempre é possível escrever a exponencial $e^{x+y}$ em uma forma aberta

$$
e^{x+y}=e^{x} e^{y} e^{C_{1}} e^{C_{2}} \ldots
$$

(chamada BCH: Baker-Campbel-Hausdorff) onde $C_{1}, C_{2}, \ldots$ são também elementos de $A$ e podem ser determinados em termos dos comutadores na álgebra. Por exemplo, é bem conhecido que

$$
C_{1}=-\frac{1}{2}[x, y]
$$

Todos os fatores $C_{k}$ são dados em termos de comutadores. Portanto, a sequência em (2.65) será finita se a álgebra for nilpotente. Esse é o caso do oscilador harmônico, onde os operadores de criação e destruição $a^{\dagger}$ e $a$ obedecem à relação de comutação $\left[a, a^{\dagger}\right]=1$. Nesse caso temos a fórmula $\mathrm{BCH}$ usual

$$
e^{\beta a^{\dagger}+\alpha a}=e^{\frac{1}{2} \alpha \beta} e^{\beta a^{\dagger}} e^{\alpha a}
$$

que é tão útil na definição dos estados coerentes.

As raízes positivas (assim como as negativas) de uma álgebra de Lie formam uma subálgebra nilpotente, que aparece na decompoisção de Gauss (2.64). Podemos portanto abrir a decomposição de Gauss até que restem apenas exponenciais simples. O segundo termo, por exemplo, será

$$
C_{2}=-\frac{1}{3}[[x, y], y]-\frac{1}{6}[[x, y], x]
$$


Um método para se obter de forma sistemática os diversos $C_{k}$ pode ser encontrado em $[40]$.

\subsubsection{Os estados coerentes}

O objeto que nos interessa aqui é o quociente, $X$, do grupo de simetria do sistema pelo subgrupo de isotropia do estado fundamental. Levando em conta a decomposição de Cartan pode-se mostrar que o espaço quociente é obtido através de uma aplicação exponencial:

$$
X(\tau)=G / H_{0}=\exp \left\{\sum_{\alpha} \tau_{\alpha} E_{\alpha}-\tau_{\alpha}^{*} E_{-\alpha}\right\}
$$

onde a soma não inclui os índices $\beta$ contidos em (2.63). Os estados coerentes serão dados pela ação de elementos desse espaço no estado fundamental:

$$
|z\rangle=X(\tau)|0\rangle=\frac{1}{\sqrt{N\left(z, z^{*}\right)}} e^{\sum_{\gamma} z_{\gamma} E_{\gamma}}|0\rangle=\frac{1}{\sqrt{N\left(z, z^{*}\right)}} \prod_{\sigma} e^{\xi_{\sigma} E_{\sigma}}|0\rangle .
$$

Note que a unitariedade do operador $X(\tau)$ garante a normalização dos estados $|z\rangle$.

A relação

$$
\exp \left\{\sum_{\alpha} \tau_{\alpha} E_{\alpha}-\tau_{\alpha}^{*} E_{-\alpha}\right\}|0\rangle=\frac{1}{\sqrt{N\left(z, z^{*}\right)}} \exp \left\{\sum_{\gamma} z_{\gamma} E_{\gamma}\right\}|0\rangle
$$

é garantida pela decomposição de Gauss, e a existência de variáveis $\xi_{\sigma}$ tais que a relação

$$
\exp \left\{\sum_{\gamma} z_{\gamma} E_{\gamma}\right\}=\prod_{\sigma} \exp \left\{\xi_{\sigma} E_{\sigma}\right\}
$$

se verifica é garantida pela fórmula BCH. Qualquer das expressões presentes na equação (2.70) serve como definição de estados coerentes.

É também comum encontrarmos na literatura uma definição de estados coe- 
rentes não-normalizados dados por

$$
\| z\rangle=\exp \left\{\sum_{\gamma} z_{\gamma} E_{\gamma}\right\}|0\rangle .
$$

\subsubsection{Supercompleteza}

Os estados coerentes são supercompletos e fornecem uma resolução da identidade:

$$
\int_{G / H} d \mu_{H}\left(z, z^{*}\right)|z\rangle\left\langle z \mid=\int_{G / H} d \mu\left(z, z^{*}\right) \| z\right\rangle\langle z \|=\mathbf{1},
$$

onde $d \mu_{H}\left(z, z^{*}\right)$ é a medida de Haar sobre $G / H[6]$ e vale a relação

$$
d \mu_{H}\left(z, z^{*}\right)=N\left(z, z^{*}\right) d \mu\left(z, z^{*}\right) .
$$

A supercompleteza implica que qualquer estado quântico pode ser escrito como combinação linear de estados coerentes:

$$
\left.|\psi\rangle=\int_{G / H} d \mu\left(z, z^{*}\right) f\left(z^{*}\right) \| z\right\rangle, \quad f\left(z^{*}\right)=\langle z \| \mid \psi\rangle .
$$

As funções $f(z)$ formam um espaço de Hilbert quadrado-integrável $L^{2}(G / H)$, e são muitas vezes chamadas "representação de Bargman" do vetor $|\psi\rangle[1,41]$. A unicidade da expansão (2.76) implica em

$$
f(x)=\int_{G / H} K\left(x, y^{*}\right) f(y) d \mu(y)
$$

onde a função $K\left(x, y^{*}\right)=\langle y\|\| x\rangle$ é um núcleo reprodutor, análogo à função delta de Dirac. É óbvio que $N\left(z, z^{*}\right)=K\left(z, z^{*}\right)$.

A resolução da identidade permite que escrevamos, para um dado operador A,

$$
A=\int_{G / H} \mathcal{A}\left(z, z^{*} ; \tilde{z}, \tilde{z}^{*}\right)|z\rangle\langle\tilde{z}| d \mu_{H}(z) d \mu_{H}(\tilde{z})
$$


e essa expressão pode, em alguns casos [42], ser colocada em uma forma diagonal, chamada representação-P do operador:

$$
A=\int A_{P}\left(z, z^{*}\right)|z\rangle\langle z| d \mu_{H}(z)
$$

Entretanto, esta representação em geral não é única. A função $A_{P}\left(z, z^{*}\right)$ também é chamada símbolo maior do operador $A$. Voltaremos a ele no Capítulo 4.

\subsubsection{Limite clássico}

A métrica no espaço coset pode ser escrita explicitamente em termos da gran$\operatorname{deza} K\left(z, z^{*}\right)[43]$ :

$$
g_{\mu \nu}=\frac{\partial^{2}}{\partial z_{\mu} \partial z_{\nu}^{*}} \ln \left[K\left(z, z^{*}\right)\right]
$$

Variedades nas quais a métrica deriva de uma função (potencial) são ditas Kählerianas, e a função $\ln \left[K\left(z, z^{*}\right)\right]$ é o potencial de Kähler neste caso.

A métrica induz de forma natural uma 2-forma fechada não-degenerada [44, $45]$

$$
\omega^{2}=i \hbar \sum_{\mu \nu} g_{\mu \nu} d z_{\mu} \wedge d z_{\nu}^{*}
$$

e um parêntese de Poisson sobre $G / H$ dado por

$$
\{u, v\}=\frac{1}{i \hbar} \sum_{\mu \nu} g^{\mu \nu}\left\{\frac{\partial u}{\partial z_{\mu}} \frac{\partial v}{\partial z_{\nu}^{*}}-\frac{\partial u}{\partial z_{\nu}^{*}} \frac{\partial v}{\partial z_{\mu}}\right\}
$$

onde $u$ e $v$ são funções definidas sobre $G / H$ e $g^{\mu \nu} g_{\nu \gamma}=\delta_{\gamma}^{\mu}$.

Como vimos na seção 2.3.1, dados $X$ e $Y$ dois elementos da álgebra, o parêntese de Poisson de seus valóres médios no estado coerentes está relacionado ao seu comutador por

$$
\hbar\{\langle z|X| z\rangle,\langle z|Y| z\rangle\}=i\langle z|[X, Y]| z\rangle,
$$

e encontramos assim um limite clássico bem definido para sistemas quânticos 
cujos observáveis possam ser escritos em termos dos geradores de uma álgebra de Lie (associada a um grupo compacto).

Se tomarmos o valor médio da equação de movimento de Heisenberg

$$
\left\langle z\left\|\frac{d X}{d t}\right\| z\right\rangle=\frac{i}{\hbar}\langle z\|[H, X]\| z\rangle
$$

onde $H$ é a Hamiltoniana e $X$ um elemento da álgebra, e multiplicarmos os dois lados por $\hbar$, o limite clássico estará bem definido e será dado por

$$
\frac{d \mathcal{X}}{d t}=\{\mathcal{H}, \mathcal{X}\}
$$

onde $\mathcal{H}$ e $\mathcal{X}$ são os limites clássicos dos operadores correspondentes. Além disso, valores médios de produtos fatoram $[8,11]$

$$
\left\langle z\left\|\hbar X_{1} \hbar X_{2}\right\| z\right\rangle \rightarrow\left\langle z\left\|\hbar X_{1}\right\| z\right\rangle\left\langle z\left\|\hbar X_{2}\right\| z\right\rangle, \quad \text { quando } \hbar \rightarrow 0
$$

e portanto a conexão entre comutadores e parêntese de Poisson não está mais restrita a observáveis lineares nos elementos da álgebra.

Estudaremos os estados coerentes e o limite clássico em detalhe no capítulo a seguir, no caso do grupo simplético $S p(4)$. 


\section{Capítulo 3}

\section{Estados Coerentes para o Grupo}

\section{Simplético Unitário $S p(4)$}

Neste capitulo, apresentaremos de forma detalhada os estados coerentes para o grupo simplético unitário $S p(4)$. Depois de rever sua teoria de representação, definiremos os estados coerentes para uma representação irredutível arbitrária e obteremos então o limite clássico de sistemas com simetria simplética. No caso de representações simétricas, encontraremos variáveis canônicas para o espaço de fase.

Além disso, investigaremos a integrabilidade de uma Hamiltoniana definida em termos dos geradores da ágebra $s p(4)$, no nível quântico e no nível clássico. Veremos que a um espaço de fase misto no limite clássico corresponde uma distribuição de espaçamento de níveis quânticos dada aproximadamente pela função de Berry-Robnik-Brody. Faremos aqui uma conexão com a área de caologia quântica.

\subsection{O grupo simplético}

Grupos simpléticos são os grupos de simetria naturais tanto da mecânica clássica quanto da mecânica quântica, uma vez que contêm todas as transformações 
lineares que preservam as relações canônicas entre variáveis conjugadas. $\mathrm{Na}$ mecânica clássica essas relações são dadas pelo parêntese de Poisson $(i, j=1 . . N)$

$$
\left\{q_{i}, p_{j}\right\}=\delta_{i j}
$$

Em mecânica quântica os operadores de posição $\hat{q}_{i}$ agem por multiplicação (na representação de posição) e os operadores de momentum $\hat{p}_{j}$ agem por diferenciação, e a dinâmica é determinada pelas relações de comutação $(i, j=1 . . N)$

$$
\left[\hat{q}_{i}, \hat{p}_{j}\right]=i \hbar \delta_{i j}
$$

Essas relações também podem ser escritas na forma (agora $i, j=1 . .2 N$ )

$$
\begin{aligned}
\left\{\xi_{i}, \xi_{j}\right\} & =J_{i j}, \\
{\left[\hat{\xi}_{i}, \hat{\xi}_{j}\right] } & =i \hbar J_{i j},
\end{aligned}
$$

onde $\xi=\left(q_{1}, \ldots, q_{N}, p_{1}, \ldots, p_{N}\right)^{T}$ e $J$ é a matriz $2 N \times 2 N$ dada por

$$
J=\left(\begin{array}{cc}
0 & 1 \\
-1 & 0
\end{array}\right) \text {. }
$$

O grupo simplético $S p(2 N, \mathbb{C})$ (em sua representação fundamental) é composto de todas as transformações complexas lineares que preservam a estrutura das relações (3.3). É fácil ver portanto que

$$
S p(2 N, \mathbb{C})=\left\{g \mid g J g^{T}=J\right\}
$$

Uma definição equivalente do grupo simplético é como o conjunto de transformações lineares complexas que preservam a forma bilinear $(\xi, \eta)=\xi^{T} J \eta$, onde $\xi, \eta$ são vetores em $\mathbb{C}^{2 N}$. Esse grupo complexo possui duas formas reais: 
a forma real normal (não-compacta) $S p(2 N, \mathbb{R})$, que é aquela naturalmente associada com mecânica clássica, e a forma real unitária (compacta) $S p(2 N)$, que é obtida através da intersecção com o grupo unitário

$$
S p(2 N)=S p(2 N, \mathbb{C}) \cap U(2 N)
$$

A álgebra de Lie $s p(2 N, \mathbb{C})$ é o conjunto de matrizes complexas $X$ que satisfazem $X^{T} J+J X=0$. Essas matrizes possuem a forma bloco-diagonal

$$
X=\left(\begin{array}{cc}
A & B \\
C & -A^{T}
\end{array}\right) \text {, }
$$

onde $A, B$ e $C$ são matrizes $N \times N$ complexas, $B$ e $C$ simétricas. O grupo simplético real e sua álgebra $s p(2 N, \mathbb{R})$ são obtidos restringindo-se essas matrizes ao corpo real. Por outro lado, o grupo simplético unitário e sua álgebra $s p(2 N)$ são obtidos impondo-se que $X$ seja antihermitiana, $X^{\dagger}=-X$, ou equivalentemente

$$
A^{\dagger}=-A, \quad B^{\dagger}+C=0=C^{\dagger}+B
$$

A forma de Killing é negativa definida em $s p(2 N)$, como é usual.

Além dessa teoria clássica, os grupos simpléticos encontram aplicações em campos tão distintos quanto ótica quântica [20] e o estudo do código genético [21]. Vale mencionar que elementos de matriz para representações gerais de $S p(2 N)$ não são conhecidos para $N>2$. Nós utilizamos os elementos de matriz que resultaram de uma recente investigação da cadeia canônica [46]

$$
S p(4) \supset S p(2) \times S p(2) \supset U(1) \times U(1) .
$$




\subsection{Representações irredutíveis de $s p(4)$}

A álgebra simplética 10-dimensional $s p(4)$ possui, de acordo com o esquema de Cartan-Weyl, as seguintes relações de comutação:

$$
\begin{aligned}
{\left[H_{i}, H_{j}\right] } & =0 \quad i, j=1,2 \\
{\left[H_{i}, E_{j}^{ \pm}\right] } & = \pm\left(\alpha_{j}\right)_{i} E_{j}^{ \pm} \quad i=1,2 \quad j=1 \ldots 4 \\
{\left[E_{i}^{+}, E_{i}^{-}\right] } & =\left(\alpha_{i}\right)_{1} H_{1}+\left(\alpha_{i}\right)_{2} H_{2}, \\
{\left[E_{1}^{+}, E_{2}^{+}\right] } & =\sqrt{2} E_{3}^{+}, \quad\left[E_{1}^{+}, E_{3}^{+}\right]=\sqrt{2} E_{4}^{+},
\end{aligned}
$$

onde $H_{1}$ e $H_{2}$ geram a subálgebra abeliana de Cartan e $E_{i}^{-}=\left(E_{i}^{+}\right)^{\dagger}$. As outras relações de comutação não nulas são derivadas dessas. As raízes positivas são

$$
\alpha_{1}=[1,-1], \quad \alpha_{2}=[0,2], \quad \alpha_{3}=[1,1], \quad \alpha_{4}=[2,0]
$$

Note a existência de duas $s p(2) \sim s u(2)$ álgebras geradas por $\left\{H_{1}, E_{4}^{+}, E_{4}^{-}\right\}$ e $\left\{H_{2}, E_{2}^{+}, E_{2}^{-}\right\}$. A soma direta dessas álgebras é a subálgebra maximal de $s p(4)$ que está associada com a cadeia canônica

$$
s p(4) \supset \operatorname{sp}(2) \oplus \operatorname{sp}(2) \supset u(1) \oplus u(1) .
$$

Essa cadeia pode ser usada para se obter um conjunto completo de números quânticos que etiquetam univocamente os vetores de qualquer representação irredutível de $s p(4)$ [47]. Elementos de matriz para os geradores da álgebra foram obtidos em [46] usando esses números quânticos. Apresentamos apenas os resultados principais.

Representações irredutíveis são etiquetadas por dois números inteiros, $\lambda_{1}$ e $\lambda_{2}$, com $\lambda_{1}>\lambda_{2}$. Os vetores de base análogos aos de Gelfand-Tsetlin para álgebras unitárias e ortogonais são dados por $\left|\sigma_{1}, \sigma_{2}, h_{1}, h_{2}\right\rangle$, onde $\sigma_{i}$ são inteiros positivos 
que obedecem às desigualdades

$$
\begin{aligned}
& \lambda_{1}-\lambda_{2} \leq \sigma_{1}+\sigma_{2} \leq \lambda_{1}+\lambda_{2}, \\
& \lambda_{2}-\lambda_{1} \leq \sigma_{1}-\sigma_{2} \leq \lambda_{1}-\lambda_{2},
\end{aligned}
$$

e $h_{i}=-\sigma_{i},-\sigma_{i}+2, \ldots, \sigma_{i}-2, \sigma_{i}$. Os números quânticos $\left(\sigma_{1}, \sigma_{2}\right)$ são os pesos máximos das representações irredutíveis de $s p(2) \oplus s p(2)$.

Por construção, os operadores diagonais possuem elementos de matriz simples:

$$
\begin{aligned}
H_{i}\left|\sigma_{1}, \sigma_{2}, h_{1}, h_{2}\right\rangle & =h_{i}\left|\sigma_{1}, \sigma_{2}, h_{1}, h_{2}\right\rangle, \\
J_{i}\left|\sigma_{1}, \sigma_{2}, h_{1}, h_{2}\right\rangle & =\sigma_{i}\left(\sigma_{i}+2\right)\left|\sigma_{1}, \sigma_{2}, h_{1}, h_{2}\right\rangle,
\end{aligned}
$$

onde os operadores de Casimir das subálgebras $s p(2)$ são

$$
J_{1}=H_{1}^{2}+\left[E_{4}^{+}, E_{4}^{-}\right]_{+}, \quad J_{2}=H_{2}^{2}+\left[E_{2}^{+}, E_{2}^{-}\right]_{+},
$$

$\mathrm{e}[A, B]_{+}=A B+B A$.

As ações dos operadores escada $\left\{E_{2}^{ \pm}, E_{4}^{ \pm}\right\}$são similares àquelas da álgebra de momentum angular e são dadas por

$$
\begin{aligned}
& E_{2}^{ \pm}\left|\sigma_{1}, \sigma_{2}, h_{1}, h_{2}\right\rangle=\left\{\frac{1}{2}\left(\sigma_{2} \mp h_{2}\right)\left(\sigma_{2} \pm h_{2}+2\right)\right\}^{\frac{1}{2}}\left|\sigma_{1}, \sigma_{2}, h_{1} \pm 2, h_{2}\right\rangle \\
& E_{4}^{ \pm}\left|\sigma_{1}, \sigma_{2}, h_{1}, h_{2}\right\rangle=\left\{\frac{1}{2}\left(\sigma_{1} \mp h_{1}\right)\left(\sigma_{1} \pm h_{1}+2\right)\right\}^{\frac{1}{2}}\left|\sigma_{1}, \sigma_{2}, h_{1}, h_{2} \pm 2\right\rangle
\end{aligned}
$$

Note que esses operadores não acoplam diferentes representações das subálgebras $s p(2)$. Esse não é o caso para os elementos restantes, $\left\{E_{1}^{ \pm}, E_{3}^{ \pm}\right\}$, que acoplam 
representações vizinhas. Suas ações são dadas por

$$
\begin{aligned}
E_{1}^{ \pm}\left|\sigma_{1}, \sigma_{2}, h_{1}, h_{2}\right\rangle & =A^{ \pm}\left|\sigma_{1}+1, \sigma_{2}+1, h_{1} \pm 1, h_{2} \mp 1\right\rangle \\
& \pm B^{ \pm}\left|\sigma_{1}+1, \sigma_{2}-1, h_{1} \pm 1, h_{2} \mp 1\right\rangle \\
& \mp C^{ \pm}\left|\sigma_{1}-1, \sigma_{2}+1, h_{1} \pm 1, h_{2} \mp 1\right\rangle \\
& +D^{ \pm}\left|\sigma_{1}-1, \sigma_{2}-1, h_{1} \pm 1, h_{2} \mp 1\right\rangle
\end{aligned}
$$

e

$$
\begin{aligned}
E_{3}^{ \pm}\left|\sigma_{1}, \sigma_{2}, h_{1}, h_{2}\right\rangle & =A^{\prime \pm}\left|\sigma_{1}+1, \sigma_{2}+1, h_{1} \pm 1, h_{2} \pm 1\right\rangle \\
& \pm B^{\prime \pm}\left|\sigma_{1}+1, \sigma_{2}-1, h_{1} \pm 1, h_{2} \pm 1\right\rangle \\
& \mp C^{\prime \pm}\left|\sigma_{1}-1, \sigma_{2}+1, h_{1} \pm 1, h_{2} \pm 1\right\rangle \\
& +D^{\prime \pm}\left|\sigma_{1}-1, \sigma_{2}-1, h_{1} \pm 1, h_{2} \pm 1\right\rangle,
\end{aligned}
$$

onde os coeficientes "linha" são obtidos dos outros simplesmente trocando-se o sinal de $h_{2}$ :

$$
X^{ \pm}=X^{ \pm}\left(h_{2} \rightarrow-h_{2}\right) \quad X \in\{A, B, C, D\}
$$

Pode-se mostrar [46] que os coeficientes $A^{+}$e $B^{+}$são dados por

$$
A^{+}=\sqrt{\frac{\left(\lambda_{+}-\sigma_{+}\right)\left(\lambda_{+}+\sigma_{+}+6\right)\left(\sigma_{+}-\lambda_{-}+2\right)\left(\sigma_{+}+\lambda_{-}+4\right)\left(\sigma_{1}+h_{1}+2\right)\left(\sigma_{2}-h_{2}+2\right)}{64\left(\sigma_{1}+1\right)\left(\sigma_{1}+2\right)\left(\sigma_{2}+1\right)\left(\sigma_{2}+2\right)}}
$$

e

$$
B^{+}=\sqrt{\frac{\left(\lambda_{-}+\sigma_{-}+2\right)\left(\lambda_{-}-\sigma_{-}\right)\left(\lambda_{+}-\sigma_{-}+2\right)\left(\lambda_{+}+\sigma_{-}+4\right)\left(\sigma_{1}+h_{1}+2\right)\left(\sigma_{2}+h_{2}\right)}{64\left(\sigma_{1}+1\right)\left(\sigma_{1}+2\right) \sigma_{2}\left(\sigma_{2}+1\right)}},
$$

onde $\lambda_{ \pm}=\lambda_{1} \pm \lambda_{2}$ e $\sigma_{ \pm}=\sigma_{1} \pm \sigma_{2}$. Os coeficientes $C^{+}$e $D^{+}$estão relacionados 
com os anteriores através de simples mudanças nos argumentos:

$$
\begin{aligned}
& C^{+}\left(\sigma_{1}, \sigma_{2}, h_{1}, h_{2}\right)=B^{+}\left(\sigma_{1}-1, \sigma_{2}-1,-\left(h_{1}+1\right),-\left(h_{2}-1\right)\right), \\
& D^{+}\left(\sigma_{1}, \sigma_{2}, h_{1}, h_{2}\right)=A^{+}\left(\sigma_{1}-1, \sigma_{2}+1,-\left(h_{1}+1\right),-\left(h_{2}-1\right)\right) .
\end{aligned}
$$

Além disso, os coeficientes de abaixamento podem ser obtidos dos de levantamento da seguinte forma:

$$
\begin{aligned}
& A^{-}\left(\sigma_{1}, \sigma_{2}, h_{1}, h_{2}\right)=D^{+}\left(\sigma_{1}+1, \sigma_{2}+1, h_{1}-1, h_{2}+1\right), \\
& B^{-}\left(\sigma_{1}, \sigma_{2}, h_{1}, h_{2}\right)=C^{+}\left(\sigma_{1}+1, \sigma_{2}-1, h_{1}-1, h_{2}+1\right), \\
& C^{-}\left(\sigma_{1}, \sigma_{2}, h_{1}, h_{2}\right)=B^{+}\left(\sigma_{1}-1, \sigma_{2}+1, h_{1}-1, h_{2}+1\right), \\
& D^{-}\left(\sigma_{1}, \sigma_{2}, h_{1}, h_{2}\right)=A^{+}\left(\sigma_{1}-1, \sigma_{2}-1, h_{1}-1, h_{2}+1\right) .
\end{aligned}
$$

\subsection{Escolha de coordenadas}

Um elemento geral $g$ do grupo $S p(4, \mathbb{C})$ é obtido através da aplicação exponencial

$$
g=\exp \left\{\gamma_{1} H_{1}+\gamma_{2} H_{2}+\sum_{i=1}^{4}\left(\eta_{i} E_{i}^{+}+\rho_{i} E_{i}^{-}\right)\right\},
$$

onde todas as letras gregas representam números complexos arbitrários. Como já notado, esse grupo não-compacto possui duas formas reais: uma não-compacta, denotada $S p(4, \mathbb{R})$, obtida restringindo-se todos os parâmetros anteriores ao corpo real; e uma compacta, denotada $S p(4)$, que consiste em matrizes unitárias obtidas impondo-se $\rho_{i}=-\eta_{i}^{*}$ e $\gamma_{1}, \gamma_{2} \in \mathbb{R}$. Neste trabalho consideramos apenas o caso compacto.

No que se segue utilizaremos a decomposição de Gauss de $S p(4)$ e seus espaços quocientes. Para realizar essa decomposição, devemos encontrar funções $(\tau, \tilde{\tau}, \alpha)$, dependendo apenas das coordenadas do grupo, tais que os elementos podem ser 
escritos

$$
g=\exp \left\{\sum_{i=1}^{4} \tau_{i} E_{i}^{+}\right\} \exp \left\{\alpha_{1} H_{1}+\alpha_{2} H_{2}\right\} \exp \left\{\sum_{i=1}^{4} \tilde{\tau}_{i} E_{i}^{-}\right\} .
$$

A relação entre as novas coordendas e as antigas pode em geral ser obtida através de um cálculo explícito na representação fundamental.

Além disso, a exponencial envolvendo raízes positivas (negativas) pode ser simplificada, conforme expusemos no Capítulo anterior. Vimos que se $x, y$ são elementos de uma álgebra de Lie, então

$$
e^{x+y}=e^{x} e^{y} e^{C_{1}} e^{C_{2}} \ldots
$$

onde todos os $C_{i}$ pertencem à álgebra de Lie. Um algorítmo para se calcular os coeficientes $C_{i}$ em termos de comutadores pode ser encontrado em [40]. A sequência de exponenciais é finita se a álgebra for nilpotente, que é o caso para as raízes positivas (negativas). Quando se trata de $s p(4)$ necessitamos apenas dos dois primeiros elementos

$$
C_{1}=-\frac{1}{2}[x, y], \quad C_{2}=-\frac{1}{3}[[x, y], y]-\frac{1}{6}[[x, y], x]
$$

Portanto, um elemento geral do grupo pode ser escrito em termos das novas coordenadas $(z, \tilde{z}, \beta)$ como

$$
g=\left\{\prod_{i=1}^{4} \exp z_{i} E_{i}^{+}\right\} \exp \left\{\beta_{1} H_{1}+\beta_{2} H_{2}\right\}\left\{\prod_{i=1}^{4} \exp \tilde{z}_{i} E_{i}^{-}\right\} .
$$




\subsection{Estados Coerentes}

\subsubsection{Definição}

Dada uma representação irredutível $\left[\lambda_{1}, \lambda_{2}\right]$, definimos os estados coerentes $|z\rangle$ por [48]

$$
|z\rangle=\exp \left\{z_{1} E_{1}^{+}\right\} \exp \left\{z_{2} E_{2}^{+}\right\} \exp \left\{z_{3} E_{3}^{+}\right\} \exp \left\{\frac{1}{\sqrt{2}}\left(z_{4}-z_{1} z_{3}\right) E_{4}^{+}\right\}|0\rangle
$$

onde $|0\rangle=\left|\lambda_{1}, \lambda_{2},-\lambda_{1},-\lambda_{2}\right\rangle$ é o peso mínimo. Os quatro números complexos $z_{i}$ são coordenadas para a variedade dos estados coerentes, e escolhemos $z_{4}$ de forma conveniente.

É fácil notar que em uma representação simétrica, onde $\lambda_{1}=\lambda$ e $\lambda_{2}=0$, qualquer elemento do tipo $\exp \left\{z_{2} E_{2}^{+}\right\}$deixa o peso mínimo invariante

$$
\exp \left\{z_{2} E_{2}^{+}\right\}|\lambda, 0,-\lambda, 0\rangle=|\lambda, 0,-\lambda, 0\rangle
$$

Esse vetor possui portanto $U(2)$ como seu grupo de isotropia, enquanto que nos casos não-simétricos o grupo de isotropia do peso mínimo é dado simplesmente pelo grupo $U(1) \times U(1)$ gerado pela subálgebra de Cartan (voltaremos a isso em outra seção). A fim de levar em conta essa isotropia impomos a restrição $z_{2}=0$ em (3.36) quando estivermos lidando com representaçoes simétricas. Essas representações serão analisadas em detalhe em outra seção.

\subsubsection{Ação dos elementos da álgebra nos estados coerentes}

A ação de um elemento de $s p(4)$ em um estado coerente em geral não resulta em outro estado coerente. A partir das relações de comutação e da definição (3.36), podemos encontrar uma representação diferencial para os elementos da álgebra. Não é difícil ver que os operadores de levantamento e os diagonais são 
representados por

$$
\begin{aligned}
E_{1}^{+}|z\rangle & =\left[\partial_{1}+z_{3} \partial_{4}\right]|z\rangle, \\
E_{2}^{+}|z\rangle & =\left[\partial_{2}-\sqrt{2} z_{1} \partial_{3}\right]|z\rangle, \\
E_{3}^{+}|z\rangle & =\left[\partial_{3}-z_{1} \partial_{4}\right]|z\rangle, \\
E_{4}^{+}|z\rangle & =\sqrt{2} \partial_{4}|z\rangle, \\
H_{1}|z\rangle & =\left[z_{1} \partial_{1}+z_{3} \partial_{3}+2 z_{4} \partial_{4}-\lambda_{1}\right]|z\rangle, \\
H_{2}|z\rangle & =\left[-z_{1} \partial_{1}+2 z_{2} \partial_{2}+z_{3} \partial_{3}-\lambda_{2}\right]|z\rangle,
\end{aligned}
$$

onde $\partial_{i}$ indica uma derivada com respeito a $z_{i}$. Os operadores de abaixamento são um pouco mais complicados:

$$
\begin{gathered}
E_{1}^{-}|z\rangle=\left[-z_{1}^{2} \partial_{1}+\sqrt{2}\left(z_{3}+\sqrt{2} z_{1} z_{2}\right) \partial_{2}+\left(z_{4}-z_{1} z_{3}\right) \partial_{3}-z_{1} z_{4} \partial_{4}+z_{1}\left(\lambda_{1}-\lambda_{2}\right)\right]|z\rangle \\
E_{2}^{-}|z\rangle=\left[-\sqrt{2} z_{3} \partial_{1}-2 z_{2}^{2} \partial_{2}+2 z_{2} \lambda_{2}\right]|z\rangle \\
\left.+\left(z_{3}+2 \sqrt{2} z_{1} z_{2}\right) \lambda_{2}+z_{3} \lambda_{1}\right]|z\rangle \\
E_{3}^{-}|z\rangle=\left[-\left(z_{4}+z_{1} z_{3}\right) \partial_{1}-2 z_{2}\left(z_{3}+\sqrt{2} z_{1} z_{2}\right) \partial_{2}-z_{3}^{2} \partial_{3}-z_{3} z_{4} \partial_{4}\right. \\
\frac{E_{4}^{-}}{\sqrt{2}}|z\rangle=\left[-z_{1} z_{4} \partial_{1}-\frac{1}{\sqrt{2}}\left(z_{3}+\sqrt{2} z_{1} z_{2}\right)^{2} \partial_{2}-z_{3} z_{4} \partial_{3}-z_{4}^{2} \partial_{4}+z_{4} \lambda_{1}\right. \\
\left.+z_{1}\left(z_{3}+z_{1} z_{2}\right) \lambda_{2}\right]|z\rangle
\end{gathered}
$$

Uma vez que nas representações simétricas os estados coerentes não dependem de $z_{2}$, as derivadas com respeito a essa variável desaparecem nesse caso. 


\subsubsection{Normalização}

Os estados definidos acima não estão normalizados. Para obter sua norma, devemos determinar as variáveis $\alpha$ e $F$ na seguinte equação:

$$
\begin{aligned}
\langle z \mid z\rangle & =\left\langle 0\left|D\left(z_{1}, z_{2}, z_{3},\left(z_{4}-z_{1} z_{3}\right) / \sqrt{2}\right) D^{\dagger}\left(z_{1}, z_{2}, z_{3},\left(z_{4}-z_{1} z_{3}\right) / \sqrt{2}\right)\right| 0\right\rangle \\
& =\left\langle 0\left|D^{\dagger}\left(\alpha_{1}, \alpha_{2}, \alpha_{3}, \alpha_{4}\right) \exp \left\{F_{1} H_{1}+F_{2} H_{2}\right\} D\left(\alpha_{1}, \alpha_{2}, \alpha_{3}, \alpha_{4}\right)\right| 0\right\rangle
\end{aligned}
$$

onde

$$
D^{\dagger}\left(z_{1}, z_{2}, z_{3}, z_{4}\right)=\exp \left\{z_{1} E_{1}^{+}\right\} \exp \left\{z_{2} E_{2}^{+}\right\} \exp \left\{z_{3} E_{3}^{+}\right\} \exp \left\{z_{4} E_{4}^{+}\right\}
$$

Note que a ordem dos operadores de levantamento e abaixamento foi invertida. A ação sobre o peso mínimo então se torna trivial e o termo diagonal provê a normalização requerida. O cálculo pode ser feito explicitamente na representação fundamental. O resultado final é

$$
\begin{aligned}
& \alpha_{1}=f^{-1}\left(z_{1}-z_{3}^{*} z_{4}\right)-\sqrt{2} z_{2}^{*} \alpha_{3}, \\
& \alpha_{2}=\frac{1}{\sqrt{2}}\left(g-f\left|\alpha_{1}\right|^{2}\right)^{-1}\left[\sqrt{2} z_{2}+z_{1}^{*}\left(z_{3}+\sqrt{2} z_{1} z_{2}\right)+f \alpha_{1} \alpha_{3}^{*}\right] \\
& \alpha_{3}=f^{-1}\left(z_{3}+z_{1}^{*} z_{4}\right) \\
& \alpha_{4}=\sqrt{2} f^{-1} z_{4}-\sqrt{2} \alpha_{1} \alpha_{3} \\
& F_{1}=-\ln f, \quad F_{2}=-\ln \left(g-f\left|\alpha_{1}\right|^{2}\right),
\end{aligned}
$$

onde $f$ e $g$ são números reais dados por

$$
\begin{aligned}
& f=1+\left|z_{1}\right|^{2}+\left|z_{3}\right|^{2}+\left|z_{4}\right|^{2}, \\
& g=1+2\left|z_{2}\right|^{2}+\left|z_{3}+\sqrt{2} z_{1} z_{2}\right|^{2}
\end{aligned}
$$


A norma é então determinada:

$$
\langle z \mid z\rangle=f^{\lambda_{1}}\left(g-f\left|\alpha_{1}\right|^{2}\right)^{\lambda_{2}}
$$

Conforme discutido no Capítulo anterior, é sempre possível definir, sobre a variedade dos estados coerentes, um parêntese de Poisson

$$
\{f, g\}=\frac{1}{i \hbar} \sum_{i, j} \omega^{i j}\left\{\frac{\partial f}{\partial z_{i}} \frac{\partial g}{\partial z_{j}^{*}}-\frac{\partial f}{\partial z_{j}^{*}} \frac{\partial g}{\partial z_{i}}\right\}
$$

onde $\omega^{i k} \omega_{k j}=\delta_{j}^{i}$ e os elementos $\omega_{i j}$ são dados em termos da norma

$$
\omega_{i j}=\frac{\partial^{2} \ln \langle z \mid z\rangle}{\partial z_{i} \partial z_{j}^{*}}
$$

Dados $X$ e $Y$ dois elementos de $s p(4)$, o parêntese de Poisson de seus valores médios está relacionado com seu comutador através da equação

$$
\hbar\{\langle z\|X\| z\rangle,\langle z\|Y\| z\rangle\}=i\langle z\|[X, Y]\| z\rangle,
$$

(onde $\| z\rangle$ denota os estados coerentes normalizados) e pode ser usado para se obter um limite clássico bem definido para um sistema quântico com simetria $s p(4)$.

Queremos agora obter o limite clássico para uma Hamiltoniana definida em uma representação irredutível de $S p(4)$. Esse limite corresponde a fazer $\hbar \rightarrow 0$ e simultaneamente aumentar a dimensão da representação de forma que a densidade de estados possa aumentar (de forma que passemos a um contínuo de estados). Uma vez que toda representação é identificada por dois números inteiros $\left[\lambda_{1}, \lambda_{2}\right]$, o limite clássico pode ser alcançado de maneiras diferentes [11, 49]. Podemos por exemplo considerar apenas representações simétricas, fazendo $\lambda_{1} \rightarrow \infty$ e mantendo $\lambda_{2}=0$. Ou podemos levar tanto $\lambda_{1}$ quanto $\lambda_{2}$ para infinito e manter sua 
razão $\lambda_{1} / \lambda_{2}$ constante. De qualquer forma o limite será implementado impondo-se que a constante de Planck $\hbar$ seja proporcional a $\left(\lambda_{1}+\lambda_{2}\right)^{-1}$.

Na seção seguinte tratamos o limite clássico de forma mais explícita.

\subsection{Representações simétricas}

Como já mencionado, nas representações simétricas, para as quais $\lambda_{1}=\lambda \mathrm{e}$ $\lambda_{2}=0$, temos que impor a restrição $z_{2}=0$. A norma dos estados coerentes simplifica enormemente neste caso e se reduz a

$$
\langle z \mid z\rangle=\left(1+\left|z_{1}\right|^{2}+\left|z_{3}\right|^{2}+\left|z_{4}\right|^{2}\right)^{\lambda}
$$

Essa simplicidade nos permite explorar muitas propriedades dos estados coerentes explicitamente. Usando o análogo da representação de Bargmann, derivamos a medida apropriada e a relação de completeza associada. Dessa forma introduzimos coordenadas canônicas que trazem o parêntese de Poisson à sua forma tradicional.

\subsubsection{Resolução da identidade}

Nas representações simétricas $H_{1}, H_{2}$ e um dos operadores de Casimir $J_{i}$ dados em (3.21) são suficientes para identificar todos os vetores da base. Sejam os estados coerentes escritos nessa base da forma

$$
|z\rangle=\sum \mathcal{F}\left(z_{1}, z_{3}, z_{4}\right)\left|\sigma_{1}, \sigma_{2}, h_{1}, h_{2}\right\rangle
$$

onde a soma inclui todos os vetores de base. A função $\mathcal{F}\left(z_{1}, z_{3}, z_{4}\right)$ é análoga à representação holomórfica de Bargmann [41], bem conhecida para o oscilador harmônico e o momentum angular. Podemos usar a representação diferencial dos elementos $H_{1}, H_{2}$ e $J_{2}$ para obter as seguintes equações diferenciais parciais para 
a função $\mathcal{F}\left(z_{1}, z_{3}, z_{4}\right)$ :

$$
\begin{gathered}
{\left[z_{1} \partial_{1}+z_{3} \partial_{3}+2 z_{4} \partial_{4}-\lambda\right] \mathcal{F}\left(z_{1}, z_{3}, z_{4}\right)=h_{1} \mathcal{F}\left(z_{1}, z_{3}, z_{4}\right)} \\
{\left[-z_{1} \partial_{1}+z_{3} \partial_{3}\right] \mathcal{F}\left(z_{1}, z_{3}, z_{4}\right)=h_{2} \mathcal{F}\left(z_{1}, z_{3}, z_{4}\right)} \\
{\left[\left(z_{1} \partial_{1}\right)^{2}+\left(z_{3} \partial_{3}\right)^{2}+2\left(z_{3} \partial_{1}\right)\left(z_{1} \partial_{3}\right)+2 z_{3} \partial_{3}\right] \mathcal{F}\left(z_{1}, z_{3}, z_{4}\right)=\sigma_{2}\left(\sigma_{2}+2\right) \mathcal{F}\left(z_{1}, z_{3}, z_{4}\right) .}
\end{gathered}
$$

O resultado é simples:

$$
\mathcal{F}\left(z_{1}, z_{3}, z_{4}\right)=c z_{1}^{\left(\sigma_{2}-h_{2}\right) / 2} z_{3}^{\left(\sigma_{2}+h_{2}\right) / 2} z_{4}^{\left(\sigma_{1}+h_{1}\right) / 2}
$$

onde $c$ é uma constante, cujo valor é fixado pela normalização. Em vista de (3.61), não é difícil ver que $c$ envolve apenas binomiais:

$$
c=\sqrt{\left(\begin{array}{c}
\lambda \\
\sigma_{1}
\end{array}\right)\left(\begin{array}{c}
\sigma_{2} \\
\left(\sigma_{2}+h_{2}\right) / 2
\end{array}\right)\left(\begin{array}{c}
\sigma_{1} \\
\left(\sigma_{1}+h_{1}\right) / 2
\end{array}\right)} .
$$

Agora demandamos dos estados coerentes a resolução da identidade, uma vez que essa é considerada uma de suas propriedades mais cruciais. Ela é escrita como uma integral

$$
\int d \mu(z)|z\rangle\langle z|=\mathbf{1}
$$

onde $d \mu(z)$ é a medida a ser determinada e o operador identidade $\mathbf{1}$ deve ser entendido como

$$
\mathbf{1}=\sum\left|\sigma_{1}, \sigma_{2}, h_{1}, h_{2}\right\rangle\left\langle\sigma_{1}, \sigma_{2}, h_{1}, h_{2}\right|,
$$

onde novamente somamos sobre todos os vetores de base e $\sigma_{2}=\lambda-\sigma_{1}$.

A partir da forma explícita da função $\mathcal{F}\left(z_{1}, z_{3}, z_{4}\right)$ podemos inferir a medida 
como

$$
d \mu(z)=\frac{(\lambda+1)(\lambda+2)(\lambda+3)}{\pi^{3}} \frac{d^{2} z_{1} d^{2} z_{3} d^{2} z_{4}}{\left(1+\left|z_{1}\right|^{2}+\left|z_{3}\right|^{2}+\left|z_{4}\right|^{2}\right)^{5}} .
$$

Note que $(\lambda+1)(\lambda+2)(\lambda+3)$ é a dimensão da representação. Esse resultado é bastante similar a outros anteriores envolvendo medidas de Haar de grupos de Lie [39].

Note também que a forma explícita dos estados coerentes em termos dos vetores de base nos permite derivar o produto escalar de dois estados diferentes

$$
\left\langle z \mid z^{\prime}\right\rangle=\left(1+z_{1}^{*} z_{1}^{\prime}+z_{3}^{*} z_{3}^{\prime}+z_{4}^{*} z_{4}^{\prime}\right)^{\lambda}
$$

\subsubsection{Parêntese de Poisson e coordenadas canônicas}

No caso de representações simétricas obtemos $\left(\lambda \equiv \lambda_{1}\right)$

$$
\omega_{i j}=\frac{\lambda}{f}\left(\delta_{i j}-\frac{z_{j} z_{i}^{*}}{f}\right),
$$

( $\delta_{i j}$ é a delta de Kronecker) com $i, j=1,3,4$, e a matriz inversa é dada por

$$
\omega^{i j}=\frac{f}{\lambda}\left(\delta_{i j}+z_{j} z_{i}^{*}\right) .
$$

Essa matriz pode ser usada para definir o parêntese de Poisson

$$
\{f, g\}=\frac{1}{i \hbar} \sum_{i, j=1,3,4} \omega^{i j}\left\{\frac{\partial f}{\partial z_{i}} \frac{\partial g}{\partial z_{j}^{*}}-\frac{\partial f}{\partial z_{j}^{*}} \frac{\partial g}{\partial z_{i}}\right\} .
$$

Podemos agora trazer essa expressão a uma forma diagonal, tradicional em mecânica clássica,

$$
\{f, g\}=\sum_{i=1}^{3}\left\{\frac{\partial f}{\partial q_{i}} \frac{\partial g}{\partial p_{i}}-\frac{\partial f}{\partial p_{i}} \frac{\partial g}{\partial q_{i}}\right\}
$$


definindo coordenadas canônicas

$$
\frac{q_{1}+i p_{1}}{\sqrt{2 \Xi}}=\frac{z_{1}}{\sqrt{1+\left|z_{1}\right|^{2}+\left|z_{3}\right|^{2}+\left|z_{4}\right|^{2}}}
$$

$\mathrm{e}$

$$
\frac{q_{j}+i p_{j}}{\sqrt{2 \Xi}}=\frac{z_{j+1}}{\sqrt{1+\left|z_{1}\right|^{2}+\left|z_{3}\right|^{2}+\left|z_{4}\right|^{2}}}, \quad j=2,3
$$

onde definimos a variável

$$
\Xi=\hbar \lambda
$$

adequada para o tratamento do limite clássico. Note a existência do vínculo

$$
E(q, p)=\sum_{i=1}^{3} q_{i}^{2}+p_{i}^{2} \leq 2 \Xi
$$

que expressa a compacidade do grupo $S p(4)$ e de seus espaços quociente.

Nessas coordenadas, os valores esperados dos elementos da álgebra com respeito aos estados coerentes normalizados podem ser obtidos:

$$
\begin{aligned}
& \mathcal{E}_{1}^{+}=\left\langle z\left\|\hbar E_{1}^{+}\right\| z\right\rangle=\frac{F}{2}\left(q_{1}-i p_{1}\right)+\frac{1}{2}\left(q_{2}+i p_{2}\right)\left(q_{3}-i p_{3}\right) \\
& \mathcal{E}_{2}^{+}=\left\langle z\left\|\hbar E_{2}^{+}\right\| z\right\rangle=\frac{1}{\sqrt{2}}\left(-q_{2}+i p_{2}\right)\left(q_{1}+i p_{1}\right) \\
& \mathcal{E}_{3}^{+}=\left\langle z\left\|\hbar E_{3}^{+}\right\| z\right\rangle=\frac{F}{2}\left(q_{2}-i p_{2}\right)-\frac{1}{2}\left(q_{1}+i p_{1}\right)\left(q_{3}-i p_{3}\right) \\
& \mathcal{E}_{4}^{+}=\left\langle z\left\|\hbar E_{4}^{+}\right\| z\right\rangle=\frac{F}{\sqrt{2}}\left(q_{3}-i p_{3}\right) \\
& \mathcal{H}_{1}=\left\langle z\left\|\hbar H_{1}\right\| z\right\rangle=\frac{1}{2}\left(q_{3}^{2}+p_{3}^{2}-F^{2}\right), \\
& \mathcal{H}_{2}=\left\langle z\left\|\hbar H_{2}\right\| z\right\rangle=\frac{1}{2}\left(q_{2}^{2}+p_{2}^{2}-q_{1}^{2}-p_{1}^{2}\right),
\end{aligned}
$$

onde $F=\sqrt{2 \Xi-E}$ e $\| z\rangle=\langle z \mid z\rangle^{-\frac{1}{2}}|z\rangle$. Os valores esperados das raízes negativas são obtidos simplesmente por conjugação. É fácil ver que essas funções possuem parêntese de Poisson (dado por (3.75)) compatível com as relações de comutação de $s p(4)$ e com a equação (3.60). 
O limite clássico é agora implementado fazendo $\hbar \rightarrow 0, \lambda \rightarrow \infty$ e mantendo seu produto $\hbar \lambda=\Xi$ constante. Como observáveis clássicos tomamos os valores médios acima. Note que a constante de Planck está presente do lado esquerdo de (3.80) (o lado "quântico") de forma a fornecer a unidade dimensional correta, enquanto que o lado direito (o lado "clássico") é independente dela.

\subsection{Caos quântico}

\subsubsection{Distribuição de espaçamento de níveis para sistemas classicamente caóticos}

Uma das principais abordagens da questão do caos em mecânica quântica é a relação entre o espectro de sistemas classicamente caóticos [50] e a teoria de matrizes aleatórias introduzida por Wigner, Dyson e Mehta [51]. Essa teoria supõe que a Hamiltoniana de um sistema classicamente caótico deve ser tão complicada que seu espectro tem as mesmas propriedades estatísticas do espectro de uma matriz aleatória. O único vínculo a ser respeitado são as simetrias do sistema (reversão temporal, paridade, etc) [52].

Vejamos o espectro de uma matriz aleatória $2 \times 2$ real e simétrica. Dentro do conjunto de todas essas matrizes, a chance de encontrarmos uma Hamiltoniana $H$ em particular deve ser dada por uma distribuição de probabilidade $P(H)$. Essa distribuição deve ser invariante sob ação do grupo $O(2)$, ou seja, $P\left(O H O^{T}\right)=P(H)$. Vamos impor que os 3 elementos $H_{11}, H_{12}, H_{22}$ não estejam correlacionados, ou seja, $P(H)=P_{11}\left(H_{11}\right) P_{12}\left(H_{12}\right) P_{22}\left(H_{22}\right)$. Além disso, queremos $\int d H_{11} d H_{12} d H_{22} P(H)=1$.

Seja uma transformação ortogonal infinitesimal

$$
O=\left(\begin{array}{cc}
1 & -\theta \\
\theta & 1
\end{array}\right)
$$


de forma que se $K=O H O^{T}$ então

$$
\begin{aligned}
& K_{11}=H_{11}-2 \theta H_{12}, \\
& K_{12}=H_{12}+\theta\left(H_{11}-H_{22}\right), \\
& K_{22}=H_{22}+2 \theta H_{12} .
\end{aligned}
$$

A invariância e a fatoração implicam em

$$
P(H)=P(H)\left\{1-\theta\left[2 H_{12} \frac{d \ln P_{11}}{d H_{11}}-2 H_{12} \frac{d \ln P_{22}}{d H_{22}}-\left(H_{11}-H_{22}\right) \frac{d \ln P_{12}}{d H_{12}}\right]\right\}
$$

Já que $\theta$ é arbitrário, temos

$$
\frac{1}{H_{12}} \frac{d \ln P_{12}}{d H_{12}}-\frac{2}{H_{11}-H_{22}}\left(\frac{d \ln P_{11}}{d H_{11}}-\frac{d \ln P_{22}}{d H_{22}}\right)=0 .
$$

Temos aí 3 equações, cuja solução é

$$
P(H)=C \exp \left[-A\left(H_{11}^{2}+H_{22}^{2}+2 H_{12}^{2}\right)+B\left(H_{11}+H_{22}\right)\right] .
$$

Das 3 constantes, $A$ fixa a unidade de energia, $B$ fixa o zero da energia e $C$ a normalização. Podemos escolher $B=0$ e escrever

$$
P(H)=C \exp \left[-A T r H^{2}\right]
$$

No caso de Hamiltonianas complexas com grupo de simetria $U(2)$ tudo procede analogamente e temos o mesmo resultado. Idem para o caso simplético. De fato, esse resultado não depende nem mesmo da dimensionalidade do espaço [52].

A função $P(H)$ fornece a densidade de probabilidade para os autovalores de $H$. Para isso, é necessário substituir $H_{i j}$ como parâmetros por um conjunto que contenha os autovalores. O restante dos parâmetros especifica a transformação 
que diagonaliza $H$.

Seja $H$ real e simétrica $2 \times 2$. Temos $E_{ \pm}=\frac{1}{2}\left(H_{11}+H_{22}\right) \pm \frac{1}{2} \sqrt{\left(H_{11}-H_{22}\right)^{2}+4 H_{12}^{2}}$. Queremos diagonalizar $H$ via

$$
O=\left(\begin{array}{cc}
\cos \theta & -\sin \theta \\
\sin \theta & \cos \theta
\end{array}\right) \text {. }
$$

Temos $H_{11}=E_{+} \cos ^{2} \theta+E_{-} \sin ^{2} \theta, H_{22}=E_{+} \sin ^{2} \theta+E_{-} \cos ^{2} \theta, H_{12}=\left(E_{+}-\right.$ $\left.E_{-}\right) \cos \theta \sin \theta$.

O Jacobiano é

$$
\operatorname{det} \frac{\partial\left(H_{11}, H_{12}, H_{22}\right)}{\partial\left(E_{+}, E_{-}, \theta\right)}=E_{+}-E_{-}
$$

e portanto temos (substituindo os elementos de matriz, o ângulo desaparece)

$$
P\left(E_{+}, E_{-}\right)=C\left|E_{+}-E_{-}\right| \exp \left\{-A\left(E_{+}^{2}+E_{-}^{2}\right)\right\} .
$$

Nos casos complexo e simplético temos

$$
P\left(E_{+}, E_{-}\right)=C\left|E_{+}-E_{-}\right|^{2} \exp \left\{-A\left(E_{+}^{2}+E_{-}^{2}\right)\right\}
$$

$\mathrm{e}$

$$
P\left(E_{+}, E_{-}\right)=C\left|E_{+}-E_{-}\right|^{4} \exp \left\{-A\left(E_{+}^{2}+E_{-}^{2}\right)\right\} .
$$

Uma vez que $P(s)=\int P(H) \delta(s-\Delta E) d H$ temos

$$
P(s)=C \int_{-\infty}^{\infty} d E_{+} \int_{-\infty}^{\infty} d E_{-} \delta\left(s-\left|E_{+}-E_{-}\right|\right)\left|E_{+}-E_{-}\right|^{n-1} \exp \left\{-A\left(E_{+}^{2}+E_{-}^{2}\right)\right\} .
$$

O resultado dessa integral é

$$
P(s)=A_{n} s^{n} e^{-s^{2} B_{n}},
$$


onde

$$
\left(A_{n} ; B_{n}\right)=\left\{\begin{array}{l}
\frac{\pi}{2} ; \frac{\pi}{4} \text { ortogonal }(n=1) \\
\frac{32}{\pi^{2}} ; \frac{4}{\pi} \text { unitária }(n=2) \\
\frac{2^{18}}{3^{6} \pi^{3}} ; \frac{64}{9 \pi} \text { simplética }(n=4)
\end{array}\right.
$$

Esses resultados são exatos para $N=2$. Para $N>2$ eles constituem em geral uma boa aproximação e são chamados "Wigner Surmise". O caso $N \rightarrow \infty$ também pode ser tratado analiticamente, mas é muito mais complexo.

\subsubsection{Distribuições de espaçamento de níveis para siste- mas classicamente mistos}

Na seção anterior vimos que os sistema quânticos cujo análogo clássico é caótico são divididos em 3 classes de universalidade. Para sistemas classicamente caóticos com simetria de reversão temporal (que quanticamente possuem Hamiltoniana é real e simétrica), conjectura-se [50], com forte suporte de evidências numéricas e experimentais [52], que a distribuição de espaçamento de níveis é aquele do Gaussian Orthogonal Ensemble, aproximadamente dada por

$$
P_{G O E}(S)=\frac{1}{2} \pi S e^{-\frac{1}{4} \pi S^{2}}
$$

Para sistemas cujo análogo clássico tem dinâmica mista (sistemas quaseintegráveis para os quais vale o teorema KAM, por exemplo), a distribuição $P_{G O E}(S)$ não é correta. No limite semiclássico $\hbar \rightarrow 0$ a distribuição correta é a de Berry-Robnik [53]

$$
e^{\rho S} P_{B R}(S, \rho)=\rho^{2} \operatorname{erfc}\left(\frac{1}{2} \sqrt{\pi} \bar{\rho} S\right)+\bar{\rho}\left(2 \rho+\frac{1}{2} \pi \bar{\rho}^{2} S\right) e^{-\frac{1}{4} \pi \bar{\rho}^{2} S^{2}},
$$


onde

$$
\bar{\rho}=1-\rho, \quad \operatorname{erfc}(x)=\frac{2}{\sqrt{\pi}} \int_{x}^{\infty} e^{-t^{2}} d t
$$

Essa distribuição é parametrizada por $\rho$, a fração do espaço de fase na qual o movimento é regular.

A distribuição (3.98) interpola suavemente entre a distribuição de Wigner $(\rho=0)$ e a de Poisson $(\rho=1)$

$$
P_{P}(S)=e^{-S}
$$

que é válida para sistemas classicamente integráveis. Ela é obtida assumindo uma contribuição para o espectro de tipo Wigner a partir das regiões caóticas e uma contribuição independente de tipo Poisson das regiões regulares.

Apesar de ser exata no limite semiclássico, a distribuição (3.98) nem sempre é uma boa aproximação para sistemas quânticos mais reais. De fato, antes do trabalho de Berry e Robnik, Brody já havia sugerido a distribuição que leva seu nome $[54]$

$$
P_{B}(S, \beta)=(1+\beta)\left[\Gamma\left(\frac{\beta+2}{\beta+1}\right)\right]^{1+\beta} S^{\beta} e^{-\beta S^{\beta+1}}
$$

e que é parametrizada por um número real $\beta \in[0,1]$. Analogamente a (3.98), para $\beta=1$ recupera-se a distribuição de Wigner e quando $\beta=0$ obtém-se a distribuição de Poisson. Apesar de não ser adequada para descrever sistemas no limite semiclássico (e nunca ter sido demonstrada teoricamente), a distribuição de Brody mostrou-se bastante útil do ponto de vista fenomenológico.

Entretanto, a distribuição de Berry-Robnik-Brody (BRB) é reconhecidamente ainda mais acurada [22]. Ela é obtida supondo-se uma contribuição tipo Poisson das regiões regulares e uma contribuição tipo Brody da região caótica. O resultado 
é

$$
P_{B R B}(S, \beta, \rho)=\frac{\partial^{2}}{\partial S^{2}}\left\{e^{-\rho S} Q\left(\frac{1}{1+\beta},\left[\Gamma\left(\frac{\beta+2}{\beta+1}\right)(1-\rho) S\right]^{1+\beta}\right)\right\}
$$

onde $Q$ é a função gamma incompleta

$$
Q(a, z)=\int_{z}^{\infty} e^{-t} t^{a-1} d t
$$

A distribuição BRB foi aplicada com sucesso na descrição do espectro de bilhares e mapas [22].

\subsection{Caos quântico em uma Hamiltoniana de $s p(4)$}

Vamos considerar a seguinte Hamiltoniana algébrica [55]

$$
H=H_{0}+\epsilon V=\hbar\left(E_{3}^{+}+E_{3}^{-}\right)+\epsilon \hbar^{2}\left(E_{1}^{+} E_{4}^{-}+E_{4}^{+} E_{1}^{-}\right),
$$

que consiste em uma perturbação quadrática $V$ sobre um termo linear $H_{0}$. Noso objetivo é investigar a transição da integrabilidade ao caos tanto no nível quântico quanto no nível clássico. No caso mais simples $\epsilon=0$ esse sistema possui duas constantes de movimento:

$$
\left[H_{0},\left(E_{3}^{+}\right)^{2}+\left(E_{3}^{-}\right)^{2}-2 H_{1} H_{2}\right]=0, \quad\left[H_{0}, H_{1}-H_{2}\right]=0 .
$$

A introdução do termo $V$ torna a primeira inválida, mas preserva a segunda:

$$
\left[V, H_{1}-H_{2}\right]=0
$$

O espaço de fase clássico é a variedade dos estados coerentes. O número de graus de liberdade será portanto igual a 4 se considerarmos uma representação 
não-simétricas e 3 se considerarmos uma representação simétrica. O sistema clássico deve possuir um espaço de fase misto em ambos os casos se $\epsilon>0$, já que nesse caso possui apenas uma constante de movimento, e a fração do espaço de fase onde o movimento é caótico deve aumentar $\operatorname{com} \epsilon$.

Essa afirmação é confirmada pela figura 3.1, onde vemos a distribuição de espaçamento de níveis $P(s)$ para $\epsilon=2.7$, em uma representação não-simétrica $\left(\lambda_{1}=23\right.$ e $\left.\lambda_{2}=11\right)$ e em uma simétrica $\left(\lambda_{1}=74\right.$ e $\left.\lambda_{2}=0\right)$. Uma vez que $\left[H, H_{1}-H_{2}\right]=0$, a análise foi feita em um subspaço invariante de $H_{1}-H_{2}$. Escolhemos aquele em que $H_{1}=H_{2}$, cuja dimensão é 1446 no caso não-simétrico e 1444 no simétrico. O valor efetivo da constante de Planck foi escolhido como $\hbar=2\left(\lambda_{1}+\lambda_{2}\right)^{-1}$. A linha cheia na figura 3.1 é a distribuição de Wigner (3.97).
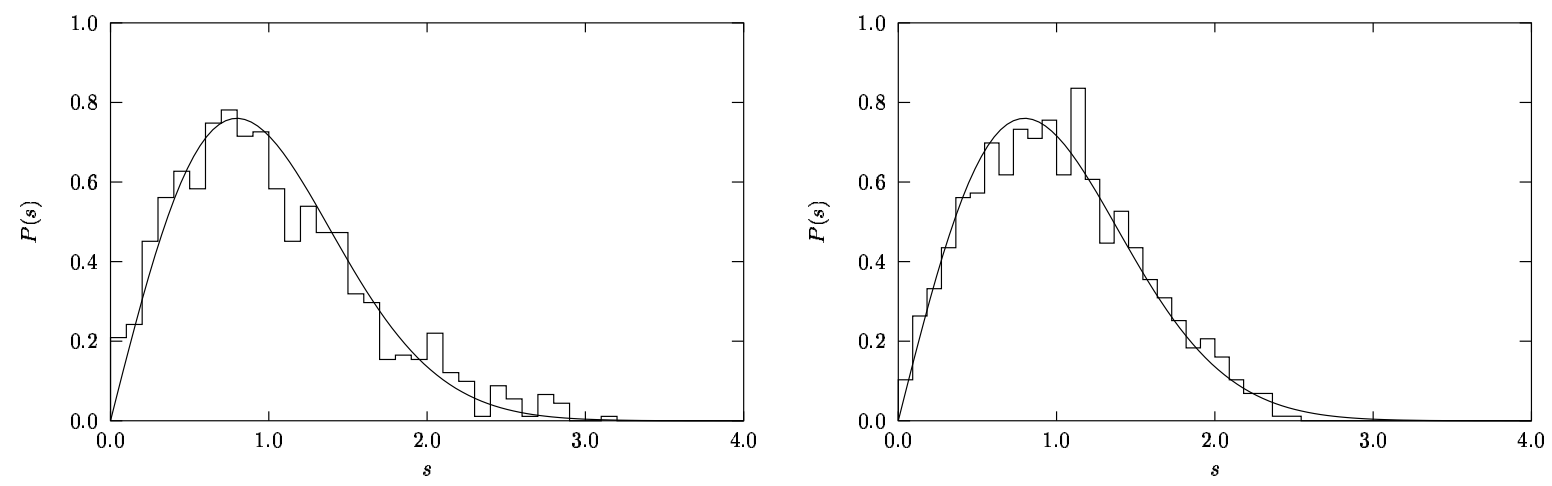

Figura 3.1: Distribuição de espaçamento de níveis para uma representação não-simétrica (esquerda) e para uma simétrica (direita). A linha cheia é a distribuição de Wigner, suposta válida para sistemas classicamente caóticos.

Consideremos agora a dinâmica clássica. Tendo em vista a figura 3.1, nos restringimos a representações simétricas, caracterizadas por um único número inteiro $\lambda$, já que neste caso possuímos as coordenadas canônicas. O limite clássico de nossa Hamiltoniana pode ser escrito nessas coordenadas:

$$
\mathcal{H}=q_{2} F-\left(q_{1} q_{3}+p_{1} p_{3}\right)+\epsilon \frac{F}{\sqrt{2}}\left[F\left(q_{1} q_{3}+p_{1} p_{3}\right)+q_{2}\left(q_{3}^{2}+p_{3}^{2}\right)\right] .
$$

É importante notar que essa Hamiltoniana possui parêntese de Poisson nulo com 
a função

$$
\mathcal{H}_{1}-\mathcal{H}_{2}=\left(q_{1}^{2}+p_{1}^{2}+q_{3}^{2}+p_{3}^{2}-\Xi\right)
$$

(onde $\mathcal{H}_{i}$ é o limite clássico de $H_{i}$ ) e portanto devemos levar em conta a conservação dessa quantidade. Podemos nos restringir ao subspaço no qual $\mathcal{H}_{1}-$ $\mathcal{H}_{2}$ é constante, efetuando uma redução dimensional que preserve a estrutura simplética. Isso será feito em dois passos. Primeiro, introduzimos as variáveis

$$
I_{i}=\frac{q_{i}^{2}+p_{i}^{2}}{2}, \quad \theta_{i}=\arctan \left(q_{i} / p_{i}\right)
$$

através de uma transformação canônica gerada pela função

$$
G_{1}=-\frac{1}{2} \sum_{i=1}^{3} p_{i}^{2} \tan \theta_{i}
$$

Tendo obtido a Hamiltoniana nas novas coordenadas, fazemos uso de uma segunda transformação canônica, dessa vez gerada por

$$
G_{2}=-I_{1} Q_{1}-I_{2} Q_{2}-\left(I_{1}+I_{3}\right) Q_{3}
$$

e o resultado final é

$$
\begin{aligned}
\mathcal{H} & =\sqrt{2 P_{2}} F \sin \left(Q_{2}\right)-2 \sqrt{P_{1}\left(P_{3}-P_{1}\right)} \cos \left(Q_{1}\right) \\
& +\epsilon F\left[F \sqrt{2\left(P_{3}-P_{1}\right) P_{1}} \cos \left(Q_{1}\right)+2\left(P_{3}-P_{1}\right) \sqrt{P_{2}} \sin \left(Q_{2}\right)\right]
\end{aligned}
$$

onde $F=\sqrt{2 \Xi-2 P_{2}-2 P_{3}}$. Note que a quantidade conservada é agora proporcional a $P_{3}$ :

$$
\mathcal{H}_{1}-\mathcal{H}_{2}=\left(2 P_{3}-\Xi\right)
$$

Note também que $Q_{3}$ não aparece na Hamiltoniana, como requerido para a conservação de seu momento conjugado. 
A quantidade $P_{3}$ pode agora ser vista como um parâmetro constante, e podemos restringir a evolução do sistema à variedade na qual $P_{3}=\Xi / 2$, que corresponde à escolha $H_{1}-H_{2}=0$ que fizemos no caso quântico. Da mesma maneira, escolhemos $\Xi=2$ e assim obtemos

$$
\begin{aligned}
\mathcal{H} & =2 \sqrt{P_{2}\left(1-P_{2}\right)} \sin \left(Q_{2}\right)-2 \sqrt{P_{1}\left(1-P_{1}\right)} \cos \left(Q_{1}\right) \\
& +\epsilon 2 \sqrt{2}\left[\left(1-P_{2}\right) \sqrt{P_{1}\left(1-P_{1}\right)} \cos \left(Q_{1}\right)+\left(1-P_{1}\right) \sqrt{P_{2}\left(1-P_{2}\right)} \sin \left(Q_{2}\right)\right] .
\end{aligned}
$$

Nas figuras 3.2(b,d,f) vemos seções de Poincaré do sistema para energia igual a -2 . A seção coincide com o plano $Q_{1}=\pi$. A trajetória clássica foi determinada propagando-se numericamente várias condições iniciais com um método RungeKutta, e apenas quando a trajetória cruzava a seção na mesma direção o ponto foi aceito. Para o pequeno valor $\epsilon=1.1$ o movimento é regular na maior parte do espaço de fase, mas ainda assim vemos uma pequena região caótica. Conforme aumentamos $\epsilon$ para 1.9 a região caótica se torna dominante, e o movimento regular fica restrito a ilhas de estabilidade. Quando $\epsilon=2.7$, situação em que $V$ e $H_{0}$ têm aproximadamente a mesma magnitude, o sistema se torna quase completamente caótico.

Uma vez que $\hbar=2 / 74$ nesse caso, estamos no regime semiclássico "próximo". Portanto não podemos esperar que a distribuição de Berry-Robnik forneça uma boa aproximação para a real distribuição de espaçamento de níveis. Em vez disso, consideramos a função de Berry-Robnik-Brody, que leva em conta não só as regiões regulares no espaço de fase no parâmetro $\rho$ mas também a localização de estados aóticos nas regiões caóticas, capturada pelo parâmetro $\beta$ [23].

Nas figuras 3.2(a,c,e) vemos a distribuição de espaçamento de níveis obtida da Hamiltoniana quântica, novamente no subspaço $H_{1}=H_{2}$. No caso fortemente 


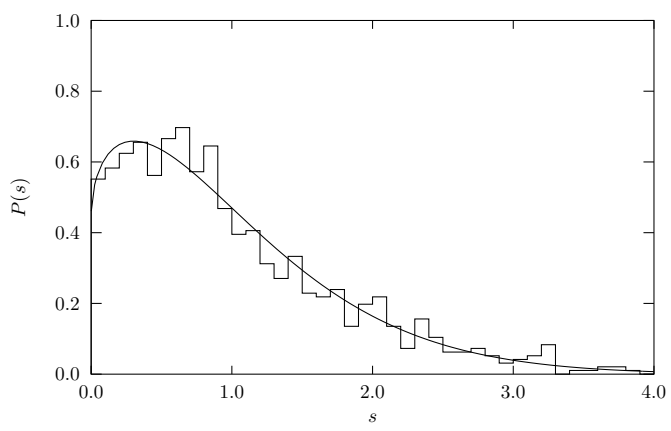

(a) $\epsilon=1.1$

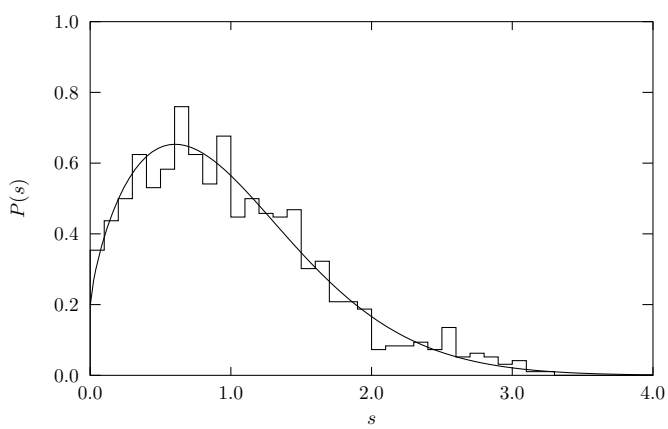

(c) $\epsilon=1.9$

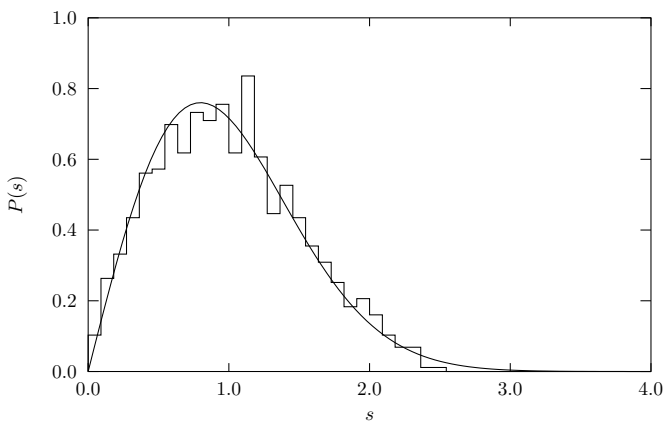

(e) $\epsilon=2.7$

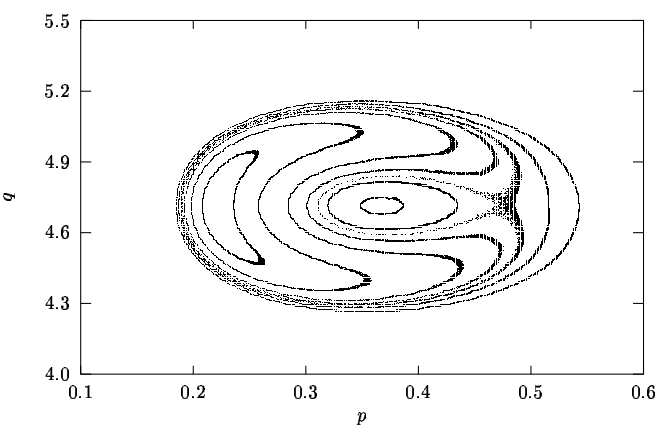

(b) $\epsilon=1.1$

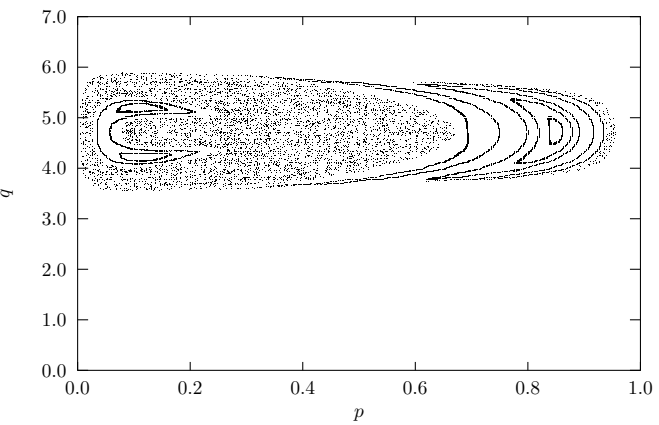

(d) $\epsilon=1.9$

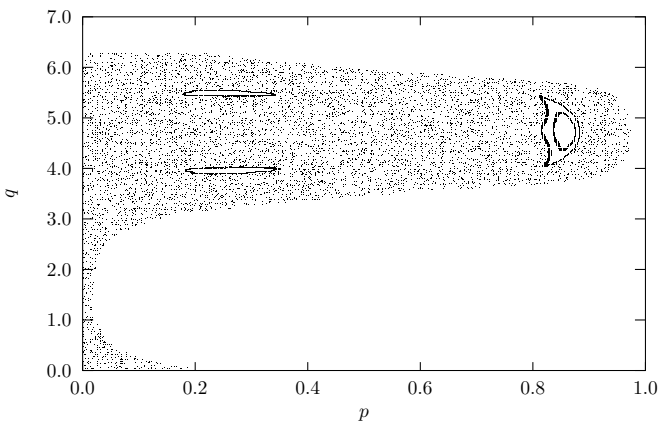

(f) $\epsilon=2.7$

Figura 3.2: Na coluna da esquerda vemos a distribuição de espaçamento de níveis para diferentes valores de $\epsilon$. A linha cheia é em (a) e (c) é a distribuição de Berry-Robnik-Brody, e em (e) é a distribuição de Wigner. Na coluna direita vemos as correspondentes seções de Poincaré. Acompanhamos assim a transição da integrabilidade para o caos nos níveis quântico e clássico.

caótico $\epsilon=2.7$ o histograma é bem aproximado pela distribuição de Wigner, como esperado. Para $\epsilon=1.9$ e $\epsilon=1.1$ a linha cheia é a distribuição de BerryRobnik-Brody (3.102). Os parâmetros foram tomados como $\beta=0.7, \rho=0.1$ para $\epsilon=1.9$ e $\beta=0.5, \rho=0.2$ para $\epsilon=1.1$. Não fizemos nenhum esforço para 
otimizar a concordância. Em acordo com a teoria, vemos que conforme o sistema se torna mais caótico $\beta$ tem que decrescer enquanto $\rho$ tem de crescer. Note que a quantidade relativamente pequena de caos presente quando $\epsilon=1.1$ é responsável por uma repulsão de níveis significativa $(P(0) \simeq 0.55$ nesse caso).

Finalmente, na figura 3.3 apresentamos a função $W(s)=\int_{0}^{s} P(x) d x$, que não sofre da mesma arbitrariedade de amostragem que $P(s)$. A linha cheia vem dos dados numéricos e a linha tracejada é derivada da predição teórica de BerryRobnik-Brody. A concordância entre as duas para $\epsilon=1.1$ e $\epsilon=1.9$ é muito boa mesmo sem nenhum procedimento de otimização. A linha pontilhada corresponde à distribuição de Wigner e é portanto característica de sistemas completamente caóticos. Essa figura dá suporte à idéia que a distribuição BRB é a melhor aproximação para sitemas com espaço de fase misto no regime semiclássico próximo.
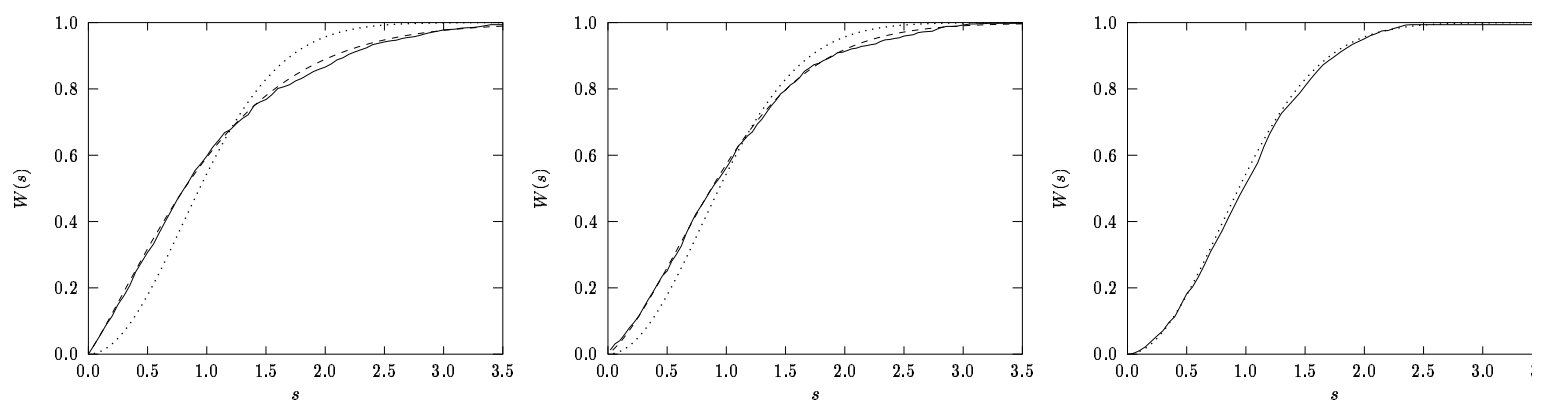

Figura 3.3: A função $W(s)$, definida no texto. Da esquerda para a direita, temos $\epsilon=1.1$, 1.9 e 2.7. A linha cheia são dados numéricos, a linha pontilhada é derivada da distribuição de Berry-Robnik-Brody, e a linha pontilhada vem da distribuição de Wigner.

\subsection{O subgrupo maximal $S p(2) \times S p(2)$}

A álgebra do primeiro subgrupo $S p(2)$ consiste em $\left\{H_{1}, E_{4}^{+}, E_{4}^{-}\right\}$. O outro subgrupo $S p(2)$ é gerado por $\left\{H_{2}, E_{2}^{+}, E_{2}^{-}\right\}$. Portanto, os elementos restantes de 
$s p(4)$ geram o coset:

$$
S p(4) /[S p(2) \times S p(2)]=M(\eta)=\exp \left\{\eta_{1} E_{1}^{+}+\eta_{2} E_{3}^{+}-\eta_{1}^{*} E_{1}^{-}-\eta_{2}^{*} E_{3}^{-}\right\} .
$$

Na representação fundamental $4 \times 4$ temos

$$
\eta_{1} E_{1}^{+}+\eta_{2} E_{3}^{+}-\eta_{1}^{*} E_{1}^{-}-\eta_{2}^{*} E_{3}^{-}=\left(\begin{array}{cc}
0 & B \\
-B^{\dagger} & 0
\end{array}\right),
$$

onde $B$ é uma matriz $2 \times 2$ dada por

$$
B=\left(\begin{array}{cc}
-\eta_{1} & -\eta_{2}^{*} \\
\eta_{2} & -\eta_{1}^{*}
\end{array}\right)
$$

A exponencial pode ser feita facilmente, e obtemos

$$
M(\eta)=\left(\begin{array}{cc}
\mathbf{1} \cos |\eta| & Z \\
-Z^{\dagger} & \mathbf{1} \cos |\eta|
\end{array}\right)
$$

onde 1 é a identidade $2 \times 2,|\eta|^{2}=\left|\eta_{1}\right|^{2}+\left|\eta_{1}\right|^{2}$ e $Z$ é dado por

$$
Z=\frac{\sin |\eta|}{|\eta|} B
$$

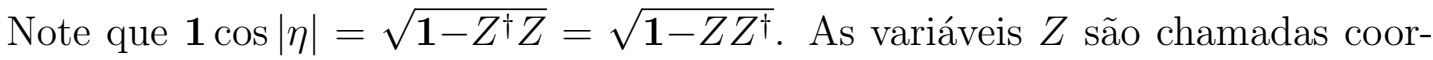
denadas projetivas.

Estados coerentes $|\eta\rangle$ são a órbita do vetor de referência $|0\rangle$ sob ação do espaço quociente $G / H,|\eta\rangle=M(\eta)|0\rangle$. Para construir estados coerentes que sejam parametrizados por pontos em $S p(4) /[S p(2) \times S p(2)]$, precisamos de um estados de referência $|0\rangle$ que tenha $S p(2) \times S p(2)$ como grupo de isotropia. Estados com essa propriedade aparecem apenas em representações antisimétricas (nas quais $\left.\lambda_{1}=\lambda_{2} \equiv \lambda\right)$, e na notação usada até agora são dados por $|0,0,0,0\rangle$. Esse estado 
é aniquilado não apenas por todas as raízes longas $\left\{E_{2}^{ \pm}, E_{4}^{ \pm}\right\}$, mas também pelos operadores diagonais $H_{1}$ and $H_{2}$ :

$$
H_{1}|0\rangle=H_{2}|0\rangle=0 \text {. }
$$

Este fato tem uma consequência importante. O valor médio de qualquer elemento $A$ da álgebra no estado coerente é dado por

$$
\langle\eta|A| \eta\rangle=\left\langle 0\left|M^{-1}(\eta) A M(\eta)\right| 0\right\rangle
$$

onde usamos a unitariedade da representação. Uma vez que $M^{-1} A M$ é também um elemento de $s p(4)$, ele pode ser escrito como uma combinação linear de todas as raízes. Mas o valor médio que qualquer raíz não-nula é zero em um estado da base. Portanto, fica claro a partir de (3.121) que o valor médio de qualquer elemento de $s p(4)$ será nulo no estado coerente:

$$
\langle\eta|A| \eta\rangle=0, \quad \forall A \in \operatorname{sp}(4), \quad \forall \eta \in \mathbb{C} .
$$

Portanto, estados coerentes fornecem neste caso apenas a representação trivial do grupo, na qual todos os elementos são representados pela operação identidade.

Examinemos em detalhe um exemplo particular. O caso mais simples é $\lambda=1$. Nessa representação temos

$$
\eta_{1} E_{1}^{+}+\eta_{2} E_{3}^{+}-\eta_{1}^{*} E_{1}^{-}-\eta_{2}^{*} E_{3}^{-}=\left(\begin{array}{cc}
0 & B \\
-B^{\dagger} & 0
\end{array}\right)
$$

onde $B$ é agora dada por uma coluna:

$$
B=\sqrt{2}\left(\begin{array}{llll}
\eta_{2} & \eta_{1} & -\eta_{1}^{*} & \eta_{2}^{*}
\end{array}\right)
$$


A exponencial pode ser calculada

$$
M(\eta)=\exp \left(\begin{array}{cc}
0 & B \\
-B^{\dagger} & 0
\end{array}\right)=\left(\begin{array}{cc}
\cos 2|\eta| & Z \\
-Z^{\dagger} & \sqrt{\mathbf{1 - Z ^ { \dagger } Z}}
\end{array}\right),
$$

onde agora

$$
Z=\chi(\eta) B
$$

e $\chi(\eta)=\sin (2|\eta|) /(\sqrt{2}|\eta|)$.

O estado coerente $|\eta\rangle$ é simplesmente

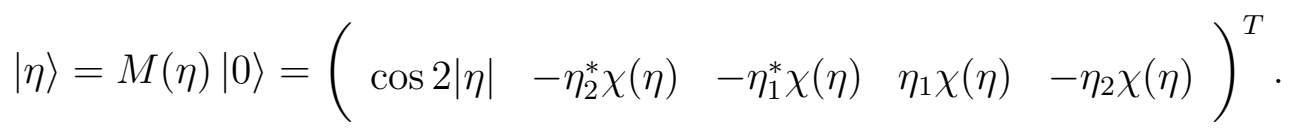

A relação (3.120) fornece as coordenadas projetivas, que são dadas por

$$
z_{i}=\frac{\eta_{i} \sin |\eta|}{|\eta|}, \quad i=1,2
$$

Nessas coordenadas, o estado coerente, agora denotado $|z\rangle$, é escrito

$$
|z\rangle=\left(\begin{array}{lllll}
1-2|z|^{2} & -z_{2}^{*} u(z) & -z_{1}^{*} u(z) & z_{1} u(z) & -z_{2} u(z)
\end{array}\right)^{T},
$$

onde $|z|^{2}=\left|z_{1}\right|^{2}+\left|z_{1}\right|^{2}$ e $u(z)=\sqrt{2-2|z|^{2}}$. É possível observar que esses estados são normalizados, $\langle z \mid z\rangle=1$. Também notamos (usando as representações matriciais) que de fato $\langle z|A| z\rangle=0$ para todo elemento $A$ de $s p(4)$.

A situação é diferente se $A$ estuver na álgebra envolvente. Os operadores de Casimir da subálgebras $s p(2)$, por exemplo, são

$$
J_{1}=H_{1}^{2}+\left[E_{4}^{+}, E_{4}^{-}\right]_{+}, \quad J_{2}=H_{2}^{2}+\left[E_{2}^{+}, E_{2}^{-}\right]_{+},
$$


e seus valores médios são simples:

$$
\mathcal{J}_{1}=\mathcal{J}_{2}=|z|^{2}\left(1-|z|^{2}\right)
$$

onde $\mathcal{J}_{i}=\left\langle z\left|J_{i}\right| z\right\rangle$. A coincidência entre os valores de $\mathcal{J}_{1}$ e $\mathcal{J}_{2}$ é particular desta representação, e não acontecerá em geral. 


\section{Capítulo 4}

\section{Estados Coerentes de}

\section{Gazeau-Klauder}

Neste capítulo será abordada uma generalização do conceito de estados coerentes que foi introduzida recentemente por John R. Klauder e Jean-Pierre Gazeau. Esses estados, chamados de Gazeau-Klauder (GK), são definidos com base apenas nos níveis de energia e não dependem de qualquer formulação em termos de grupos de Lie.

Introduzir os estados GK para um gás de elétrons bidimensional e para o potencial de mínimos duplos unidimensional. O primeiro caso é uma ilustração do formalismo para mais de um grau de liberdade, e utilizamos diferentes simetrias do problema para definir famílias de estados GK. Já o segundo caso exigiu extensas simulações numéricas pelo fato do potencial não possuir soluções exatas. Entretanto, concluímos que no limite de altas energias os estados GK têm um comportamento simples, associados aos chamados gatos de Schrödinger. 


\subsection{Introdução}

Os estados coerentes do oscilador harmônico,

$$
|\alpha\rangle=e^{-|\alpha|^{2} / 2} \sum_{n=0}^{\infty} \frac{\alpha^{n}}{\sqrt{n !}}|n\rangle,
$$

possuem uma série de propriedades notáveis, conforme exposto anteriormente. Além de serem autoestados do operador de aniquilação, eles estão associados com a órbita coadjunta do peso mínimo, minimizam a relação de incerteza de Heisenberg, e são supercompletos. Todas essas propriedades serviram de guia para diferentes generalizações: a primeira e a segunda dependem de algum tipo de estrutura algébrica por trás do sistema em questão [6], enquanto as duas últimas são mais gerais.

Neste capítulo apresentamos uma proposta recente de estados coerentes generalizados [24], os estados de Gazeau-Klauder, que parece ser a mais geral, impondo apenas normalização, continuidade nos parâmetros e completeza (também chamada resolução da identidade). Além dessas três demandas básicas, quando lidamos com sistemas físicos costumamos requerer estabilidade temporal, o sentido de que a evolução temporal de um estado coerentes é um outro estado coerente.

Esses estados foram construídos recentemente para o oscilador de Poschl-Teller unidimensional (do qual o poço quadrado é um caso particular) [26], onde a presença de soluções exatas em forma analítica permite uma análise completa do problema. Outros exemplos são o oscilador não linear que aparece em [56] e o cálculo apresentado em [57] para um potencial periódico, onde os estados GK são comparados com estados coerentes canônicos. A relação entre esses estados e relações de incerteza generalizadas foi explorada em [58], e um parêntese de Moyal adequado foi construído em [59].

Na primeira parte deste capítulo apresentamos uma extensão dos estados GK para mais de uma dimensão. Essa etapa do trabalho foi realizada em colaboração 
com o Prof. Jean-Pierre Gazeau, da Universidade de Paris 7. O formalismo resultante será então aplicado a um problema termodinâmico bidimensional, usando as desigualdades de Berezin-Lieb.

Na segunda parte tratamos o potencial de mínimos duplos unidimensional. Esse potencial tem recebido muita atenção ao longo dos anos devido à sua similaridade com a teoria quântica de campos $\phi^{2}+\lambda \phi^{4}$, que é o protótipo da quebra espontânea de simetria. Por outro lado, a aplicação de um campo externo dependente do tempo que torna o sistema caótico faz possível seu uso na investigação das relações entre tunelamento e caos [60]. Além disso, nós concluímos que o estados GK desse sistema estão relacionados e Gatos de Schödinger para altas energias, revelando assim outra propriedade interessante desse potencial.

\subsection{Estados coerentes de Gazeau-Klauder}

Apresentamos nesta seção a teoria de Gazeau e Klauder de estados coerentes para potenciais gerais [24]. Seja $H$ uma Hamiltoniana com espectro puramente discreto e não-degenerado (finito ou infinito),

$$
H|n\rangle=\hbar \omega e_{n}|n\rangle, n \geq 0
$$

onde os $e_{n}$ são números puros e $e_{0}=0$. Usaremos unidades nas quais $\hbar=\omega=1$. Os estados coerentes generalizados são dados por

$$
|J, \gamma\rangle=\frac{1}{\sqrt{N(J)}} \sum_{n \geq 0} \frac{J^{n / 2}}{\sqrt{\rho_{n}}} e^{-i e_{n} \gamma}|n\rangle
$$

onde $0 \leq J \in \mathbb{R}, \gamma \in \mathbb{R}$, e $\rho_{n}$ é uma função real positiva de $n$ com $\rho_{0}=1$. Esta é uma generalização direta de (4.1), que corresponde a $e_{n}=n$ e $\rho_{n}=n$ !. 
A condição de normalização impõe que a série

$$
N(J)=\sum_{n \geq 0} \frac{J^{n}}{\rho_{n}}
$$

seja convergente para algum raio $R$ (que pode ser infinito), e por construção temos a propriedade de estabilidade temporal:

$$
e^{-i H t}|J, \gamma\rangle=|J, \gamma+t\rangle
$$

Além disso, se o problema de momentos

$$
\int_{0}^{R} J^{n} \frac{W(J)}{N(J)} d J=\rho_{n}
$$

admitir uma solução positiva para $W(J)$, então existe uma resolução da identidade em termos desses estados, no sentido de que:

$$
\lim _{\Gamma \rightarrow \infty} \frac{1}{2 \Gamma} \int_{-\Gamma}^{\Gamma} d \gamma \int_{0}^{R} d J W(J)|J, \gamma\rangle\left\langle J, \gamma\left|=\sum_{n \geq 0}\right| n\right\rangle\langle n| .
$$

O valor médio da Hamiltoniana nesses estados (também chamado seu símbolo inferior ou função- $Q$ ) é determinado pela escolha da função $\rho_{n}$. Uma escolha comum, que adotaremos aqui, é $\rho_{n}=e_{1} e_{2} \ldots e_{n}$ para $n>0$, caso em que a variável $J$ pode ser interpretada como a energia média:

$$
\langle J, \gamma|H| J, \gamma\rangle=J
$$

Os coeficientes $\langle n \mid \alpha\rangle$ dos estados coerentes canônicos seguem uma distribuição de Poisson. O caráter estatístico de um estado geral é medido pelo parâmetro de Mandel [61]

$$
Q=\frac{(\Delta n)^{2}}{\langle n\rangle}-1=\frac{\left\langle n^{2}\right\rangle-\langle n\rangle^{2}}{\langle n\rangle}-1
$$


que fornece uma estimativa de quantos estados $|n\rangle$ efetivamente contribuem para a superposição (4.3). Um estado poissoniano, ou seja, com $(\Delta n)^{2}=\langle n\rangle$, possui $Q=0$. Os casos $Q<0$ e $Q>0$ correspondem a estados ditos sub-poissonianos e super-poissonianos respectivamente. No caso dos estados GK temos

$$
\langle n\rangle=J \frac{d}{d J} \ln N(J)
$$

e portanto $Q$ é independente de $\gamma$ e dado por

$$
Q(J)=J \frac{d}{d J} \ln \left[\frac{d}{d J} \ln N(J)\right]
$$

É fácil ver que para pequenos valores de $J$ podemos aproximar essa expressão por

$$
Q(J) \simeq J\left(\frac{2}{e_{2}}-\frac{1}{e_{1}}\right)<0, \quad J \ll 1
$$

\subsubsection{Exemplos}

Devemos notar que todos os casos a seguir têm em comum a natureza linear do espectro, o que permite um agrupamento das variáveis $J$ e $\gamma$ e leva a uma formulação analítica do tipo Fock-Bargmann.

\section{1) Oscilador harmônico}

O espaço de estados do oscilador harmônico é um espaço de Hilbert de dimensão infinito no qual a equação de Schrödinger estacionária se escreve $(\hbar=1)$

$$
H|n\rangle=\omega n|n\rangle
$$


(consideramos uma hamiltoniana deslocada para zerar a energia de ponto zero). Tendo isso em conta, a equação (4.3) fica

$$
|J, \gamma\rangle=e^{-J / 2} \sum_{n=0}^{\infty} \frac{J^{n / 2}}{\sqrt{n !}} e^{-i n \gamma}|n\rangle .
$$

Identificando $\sqrt{J} e^{-i \gamma} \equiv \alpha$ temos os estados coerentes canônicos, que chamaremos estados de Glauber-Klauder-Sudarshan (GKS).

Esses estados são supercompletos com função peso $W(J)=\frac{1}{2 \pi}$ :

$$
\frac{1}{2 \pi} \int_{0}^{2 \pi} d \gamma \int_{0}^{\infty} d J|J, \gamma\rangle\left\langle J, \gamma\left|=\sum_{n=0}^{\infty}\right| n\right\rangle\langle n|=\mathbf{1} .
$$

2) O caso $s u(1,1)$

Outra escolha interessante para $\rho_{n}$ é baseada no coeficiente binomial:

$$
\rho_{n}=\left(\begin{array}{c}
\nu+n \\
n
\end{array}\right)^{-1}, 1 \leq \nu \in \mathbb{N} .
$$

Neste caso temos

$$
|\nu ; J, \gamma\rangle=(1-J)^{(\nu+1) / 2} \sum_{n=0}^{\infty}\left(\begin{array}{c}
\nu+n \\
n
\end{array}\right)^{\frac{1}{2}} J^{n / 2} e^{-i n \gamma}|n\rangle
$$

e a condição de normalização impõe $0 \leq J<1$. Esses estados podem ser identificados com o estados coerentes de Perelomov para a álgebra $s u(1,1)$ em suas representações da série discreta $U^{\nu}$ onde $\nu \in \mathbb{N}^{*}[6]$. Lembramos que os geradores obedecem as relações de comutação da álgebra $s u(1,1)$

$$
\left[K_{0}, K_{ \pm}\right]= \pm K_{ \pm}, \quad\left[K_{+}, K_{-}\right]=-2 K_{0}
$$


e na série discreta temos

$$
K_{0}|\nu, n\rangle=\left(\frac{\nu+1}{2}+n\right)|\nu, n\rangle, \nu \geq 1, n \geq 0
$$

(a notação aqui é ligeiramente diferente da usual). Assim como no caso anterior, o agrupamento dos parâmetros no número complexo $z=\sqrt{J} e^{-i \gamma}$ leva a um formalismo de Fock-Bargmann. Entretanto, a importante diferença com o caso do oscilador está no fato de que $z$ agora é restrito ao disco aberto de raio 1.

A função peso para esses estados é $W(J)=\frac{\nu}{2 \pi(1-J)^{2}}$ e a relação de supercompleteza vale no disco unitário:

$$
\frac{\nu}{2 \pi} \int_{0}^{2 \pi} d \gamma \int_{0}^{1} \frac{d J}{(1-J)^{2}}|\nu ; J, \gamma\rangle\left\langle\nu ; J, \gamma\left|=\sum_{n=0}^{\infty}\right| n\right\rangle\langle n|=\mathbf{1}
$$

\section{3) O caso $s u(2)$}

Podemos também aplicar esse formalismo a um sistema de dimensão finita, por exemplo uma representação irredutível de $s u(2)$, caso no qual nós tambem temos uma estrutura complexa e vale a equação $L_{z}|j, m\rangle=m|j, m\rangle, j \in \mathbb{N} / 2,-j \leq$ $m \leq j$. A condição $e_{n} \geq 0$ demanda a introdução de um operador deslocado $\widetilde{L_{z}}=L_{z}+j$ e de estados $|n\rangle \equiv|j, m\rangle$ tais que $n=j+m$. Portanto, $\widetilde{L_{z}}|n\rangle=n|n\rangle$ e podemos escrever

$$
|z\rangle=\frac{1}{\sqrt{\mathcal{N}\left(|z|^{2}\right)}} \sum_{n=0}^{2 j} \frac{z^{n}}{\sqrt{\rho_{n}}}|n\rangle, \quad z=\sqrt{J} e^{-i \gamma}
$$

Se escolhermos

$$
\rho_{n}=\left(\begin{array}{c}
2 j \\
n
\end{array}\right)^{-1}
$$

teremos

$$
\mathcal{N}\left(|z|^{2}\right)=\sum_{n=0}^{2 j}\left(\begin{array}{c}
2 j \\
n
\end{array}\right)|z|^{2 n}=\left(1+|z|^{2}\right)^{2 j}
$$


e portanto

$$
|z\rangle=\frac{1}{\left(1+|z|^{2}\right)^{j}} \sum_{m=-j}^{j}\left(\begin{array}{c}
2 j \\
j+m
\end{array}\right)^{\frac{1}{2}} z^{j+m}|j, m\rangle,
$$

que são os estados coerentes usuais de $s u(2)$. Podemos usar pontos sobre o superfície esférica, conforme vimos no Capítulo 2, e nesse caso

$$
\frac{2 j+1}{4 \pi} \int|j ; \theta, \varphi\rangle\left\langle j ; \theta, \varphi\left|d S=\frac{2 j+1}{\pi} \int \frac{d^{2} z}{\left(1+|z|^{2}\right)^{2}}\right| z\right\rangle\left\langle z\left|=\sum_{m=-j}^{j}\right| j, m\right\rangle\langle j, m| .
$$

onde $d S=\sin \theta d \theta d \varphi$.

\subsubsection{Símbolos e desigualdades de Berezin-Lieb}

Berezin [62] e Lieb [9] introduziram separadamente os conceitos de símbolos maior e menor de um operador $A$, respectivamente $\hat{A}$ e $\check{A}$. Eles são definidos através das relações

$$
\begin{gathered}
A=\int d \mu(z) \hat{A}(z)|z\rangle\langle z|, \\
\check{A}(z)=\langle z|A| z\rangle,
\end{gathered}
$$

onde $|z\rangle$ é um conjunto (super-) completo de estados. Note que dado um operador A seu símbolo maior pode não ser bem definido. A seguir apresentamos alguns desses símbolos para os exemplos anteriores.

- Para o oscilador harmônico e em termos dos correspondentes operadores de criação e destruição,

$$
\begin{gathered}
\left\langle z\left|a^{\dagger} a\right| z\right\rangle=|z|^{2}, \\
a^{\dagger} a=\int d^{2} z\left(|z|^{2}-1\right)|z\rangle\langle z| .
\end{gathered}
$$


- Para a álgebra $s u(2)$,

$$
\begin{gathered}
\left\langle j ; \theta, \varphi\left|L_{z}\right| j ; \theta, \varphi\right\rangle=j \cos \theta \\
L_{z}=\frac{2 j+1}{4 \pi} \int d S(j+1) \cos \theta|l ; \theta, \varphi\rangle\langle j ; \theta, \varphi| .
\end{gathered}
$$

- Para a álgebra $s u(1,1)$,

$$
\begin{gathered}
\left\langle\nu ; J, \gamma\left|K_{0}\right| \nu ; J, \gamma\right\rangle=\frac{\nu+1}{2}\left(\frac{1+J}{1-J}\right), \\
K_{0}=\frac{1}{2 \pi} \int_{0}^{2 \pi} d \gamma \int_{0}^{1} \frac{\nu d J}{(1-J)^{2}}\left(\frac{\nu-1}{2}\right)\left(\frac{1+J}{1-J}\right)|\nu ; J, \gamma\rangle\langle\nu ; J, \gamma| .
\end{gathered}
$$

No caso $\nu=1$ a última equação não se aplica e deve ser substituída por

$$
K_{0}=\lim _{\epsilon \rightarrow 0^{+}} \frac{1}{2 \pi} \int_{0}^{2 \pi} d \gamma \int_{0}^{1} d J \frac{\delta(1-\epsilon-J)}{(1-J)^{2}}|\nu=1 ; J, \gamma\rangle\langle\nu=1 ; J, \gamma|
$$

Pode-se mostrar [9] que, dado um operador hermitiano $A$ e uma função convexa $g$, existem as seguintes desigualdades, chamadas desigualdades de BerezinLieb (BL):

$$
\int d \mu(z) g(\check{A}) \leq \operatorname{Tr}(g(A)) \leq \int d \mu(z) g(\hat{A})
$$

Como aplicação de nosso formalismo, apresentamos na próxima seção o cálculo das desigualdades de BL para o potencial termodinâmicos de um gás de elétrons bidimensional em um campo magnético constante perpendicular.

Neste ponto, uma questão interessante se apresenta: dado um espaço de Hilbert com uma base ortonormal $\{|n\rangle, n \in \mathbb{N}\}$, e o operador de número $N$ definido por $N|n\rangle=n|n\rangle$, podemos construir ambas as famílias de estados (4.14) e (4.17). Qual a relação entre as desigualdades de BL associadas? A resposta é uma simples mudança de coordenadas. Quando lidamos com a família (4.17) devemos 
interpretar $N$ como $K_{0}-(\nu+1) / 2$ e as desigualdades de BL são

$$
\int_{0}^{1} \frac{\nu d J}{(1-J)^{2}} g\left(\frac{(\nu+1) J}{1-J}\right) \leq \operatorname{Tr}(g(N)) \leq \int_{0}^{1} \frac{\nu d J}{(1-J)^{2}} g\left(\frac{(\nu-1) J}{1-J}-1\right)
$$

onde $g$ é qualquer função convexa. As transformações

$$
x_{ \pm}=\frac{(\nu \pm 1) J}{1-J}
$$

levam (4.36) em

$$
\frac{\nu}{\nu+1} \int_{0}^{\infty} g\left(x_{+}\right) d x_{+} \leq \operatorname{Tr}(g(N)) \leq \frac{\nu}{\nu-1} \int_{0}^{\infty} g\left(x_{-}-1\right) d x_{-}
$$

É evidente que se tivéssemos usado a família (4.14) teríamos obtido o mesmo resultados, exceto pela dependência em $\nu$. Essa dependência mostra que os estados coerentes canônicos são mais adequados para calcular desigualdades de BL do que qualquer das famílias (4.17). Uma observação similar pode ser feita para os estados coerentes de $s u(2)$ e vemos que no limite $j \rightarrow \infty$ eles fornecem os mesmos resultados dos canônicos.

\subsubsection{Generalização}

Queremos agora generalizar a expressão (4.3) para o caso de vários graus de liberdade, ou seja, quando os estados de base são escritos como $\left|n_{1}, n_{2}, \ldots, n_{r}\right\rangle \equiv$ $|\mathbf{n}\rangle, r \geq 2$.

Começamos com um exemplo, estendendo a construção para su(2) exposta anteriormente. Considere o espaço de Hilbert $\mathcal{H}=\oplus_{2 j \in \mathbb{N}} \mathcal{H}_{j}$, soma direta de todos os espaços $\mathcal{H}_{j}=\mathbb{C}^{2 j+1}$ carregadores de representações unitárias irredutíveis de $s u(2)$. Para cada representação $U^{j}$ temos uma família $\{|j ; \theta, \varphi\rangle, 2 j \in \mathbb{N}, 0 \leq$ $\theta<\pi, 0 \leq \varphi<2 \pi\}$ satisfazendo (4.25), que neste caso chamaremos "resolução do projetor ortogonal" $\mathbb{I}_{2 j+1}$. Para obter uma famílila supercompleta de estados 
que resolvam a identidade no espaço de Hilbert maior $\mathcal{H}$ podemos, por exemplo, definir

$$
\left|J_{1}, J_{2}, \gamma_{1}, \gamma_{2}\right\rangle=e^{-J_{1} / 2} \sum_{2 j \in \mathbb{N}} \frac{J_{1}^{j} e^{-i 2 j \gamma_{1}}}{\sqrt{(2 j) !}}|j ; \theta, \varphi\rangle \equiv\left|J_{1}, \gamma_{1}, \theta, \varphi\right\rangle
$$

onde $\tan (\theta / 2) e^{-i \varphi}=\sqrt{J_{2}} e^{-i \gamma_{2}}$. Devemos notar o caráter "tipo GKS" dessa superposição. Esses estados obedecem

$$
\int_{0}^{2 \pi} d \gamma_{1} \int_{0}^{\infty} d J_{1} \int d S W\left(J_{1}\right)\left|J_{1}, \gamma_{1}, \theta, \varphi\right\rangle\left\langle J_{1}, \gamma_{1}, \theta, \varphi\right|=\mathbf{1}
$$

com $W\left(J_{1}\right)=\frac{J_{1}}{8 \pi^{2}}$. Observamos que é também interessante dividir $\mathcal{H}$ nos setores bosônico e fermiônico,

$$
\mathcal{H}=\mathcal{H}_{\text {bos }} \oplus \mathcal{H}_{\text {ferm }}
$$

e apresentar os resultados explícitos para as funções peso $W_{\text {bos }}$ e $W_{\text {ferm }}$ envolvidas na resolução dos respectivos projetores $\mathbb{I}_{\text {bos }}$ e $\mathbb{I}_{\text {ferm. }}$. O resultado é

$$
\begin{aligned}
\left|J_{1}, \gamma_{1}, \theta, \varphi\right\rangle_{\text {bos }} & =e^{-J_{1} / 2} \sum_{j} \frac{J_{1}^{j / 2} e^{-i j \gamma_{1}}}{\sqrt{j !}}|j ; \theta, \varphi\rangle, \quad j=0,1,2, \ldots \\
\left|J_{1}, \gamma_{1}, \theta, \varphi\right\rangle_{\text {ferm }} & =e^{-J_{1} / 2} \sum_{j} \frac{J_{1}^{(2 j-1) / 4} e^{-i(2 j-1) \gamma_{1} / 2}}{\sqrt{\left(\frac{2 j-1}{2}\right) !}}|j ; \theta, \varphi\rangle, \quad j=\frac{1}{2}, \frac{3}{2},(.4 \\
W_{\text {bos }}\left(J_{1}\right) & =\frac{2 J_{1}-1}{8 \pi^{2}} \\
W_{\text {ferm }}\left(J_{1}\right) & =\frac{2 J_{1}}{8 \pi^{2}}
\end{aligned}
$$

Baseados nesse exemplo simples, obtemos generalizações multidimensionais dos estados coerentes tradicionais. Inicialmente, vamos assumir um conjunto completo de $r$ observáveis satisfazendo as relações de autovalores:

$$
A_{i}|\mathbf{n}\rangle=\omega_{i} e_{i}(\mathbf{n})|\mathbf{n}\rangle
$$


Então, podemos lidar com a seguinte forma geral de estados coerentes [63]:

$$
|\mathbf{J}, \gamma\rangle=\frac{1}{\sqrt{\mathcal{N}(\mathbf{J})}} \sum_{\{n\}} \frac{\mathbf{J}^{\mathbf{n} / 2}}{\sqrt{\rho(\mathbf{n})}} e^{-i \boldsymbol{\gamma} \cdot \mathbf{e}(\mathbf{n})}|\mathbf{n}\rangle
$$

onde a soma é sobre todos os valores possíveis das variáveis $n_{i}, \mathcal{N}$ é um fator de normalização e $\rho(\mathbf{n})$ é uma função positiva arbitrária de todos os índices. As expressões $\mathbf{J}^{\mathbf{n} / 2}$ e $\boldsymbol{\gamma} \cdot \mathbf{e}(\mathbf{n})$ devem ser entendidas como $\prod_{i=1}^{r} J_{i}^{n_{i} / 2}$ e $\gamma_{1} e_{1}(\mathbf{n})+\cdots+$ $\gamma_{r} e_{r}(\mathbf{n})$, respectivamente. Podemos adicionar condições específicas à definição (4.47), como foi feito por Gazeau e Klauder, mas em vez disso adotamos uma abordagem mais intuitiva, baseada em recursividade.

Introduzimos primeiro variáveis de coerência para o r-ésimo grau de liberdade:

$$
\left|n_{1}, n_{2}, \ldots, J_{r}, \gamma_{r}\right\rangle=\frac{1}{\sqrt{\mathcal{N}_{r}\left(J_{r}\right)}} \sum_{n_{r}} \frac{J_{r}^{n_{r} / 2}}{\sqrt{\rho_{r}}} e^{-i \gamma_{r} e_{r}(\mathbf{n})}|\mathbf{n}\rangle
$$

onde a soma é sobre todos os possíveis valores de $n_{r}$ e tanto a norma $\mathcal{N}_{r}\left(J_{r}\right)$ quanto a função $\rho_{r}$ podem depender dos índices restantes. Esses estados devem satisfazer a resolução do projetor ortogonal no subspaço definido fixando-se $n_{1}, n_{2}, \cdots, n_{r-1}$ (vamos impor as condições de Klauder em cada passo):

$$
\int d \mu\left(J_{r}, \gamma_{r}\right)\left|n_{1}, n_{2}, \ldots, J_{r}, \gamma_{r}\right\rangle\left\langle n_{1}, n_{2}, \ldots, J_{r}, \gamma_{r}\left|=\sum_{n_{r}}\right| \mathbf{n}\right\rangle\langle\mathbf{n}|=\mathbb{I}_{n_{1}, n_{2}, \cdots, n_{r-1}}
$$

Agora, se supusermos que a dependência dos $\rho_{i}$ 's e $e_{i}$ 's na $r$-upla $\boldsymbol{n}$ está organizada de forma hierárquica como $\rho_{i}(\boldsymbol{n})=\rho_{i}\left(n_{1}, n_{2}, \cdots, n_{i}\right)$ e $e_{i}(\boldsymbol{n})=e_{i}\left(n_{1}, n_{2}, \cdots, n_{i}\right)$, podemos proceder à associação de variáveis de coerência para cada grau de liberdade até obtermos

$$
|\boldsymbol{J}, \boldsymbol{\gamma}\rangle=\frac{1}{\sqrt{\mathcal{N}_{1}}} \sum_{n_{1}} \frac{J_{1}^{n_{1} / 2}}{\sqrt{\rho_{1}}} e^{-i \gamma_{1} e_{1}} \frac{1}{\sqrt{\mathcal{N}_{2}}} \sum_{n_{2}} \frac{J_{2}^{n_{2} / 2}}{\sqrt{\rho_{2}}} e^{-i \gamma_{2} e_{2}} \cdots \frac{1}{\sqrt{\mathcal{N}_{r}}} \sum_{n_{r}} \frac{J_{r}^{n_{r} / 2}}{\sqrt{\rho_{r}}} e^{-i \gamma_{r} e_{r}}|\mathbf{n}\rangle
$$

onde $\mathcal{N}_{i}$ representa $\mathcal{N}_{i}\left(J_{i}, J_{i+1}, \cdots, J_{r} ; n_{1}, n_{2}, \cdots, n_{i-1}\right)$. 
Equação (4.46) garante estabilidade sob ação do grupo (abeliano) gerado pelos operadores $A_{i}$. A escolha das funções $\rho_{i}$ vai determinar seus valores médios. Devemos ter em mente a possível dependência de $\rho_{i}$ e $e_{i}$ nos números quânticos $n_{j}, j<i$ e é evidente que se tal dependência não estiver presente então o resultado final será simplesmente produtos tensoriais de estados do tipo (4.3).

\section{Exemplos}

Para ilustrar o formalismo, consideremos o caso simples de dois graus de liberdade, $r=2$. Nesse caso a equação (4.50) se reduz a

$$
\left|J_{1}, J_{2}, \gamma_{1}, \gamma_{2}\right\rangle=\frac{1}{\sqrt{\mathcal{N}_{1}\left(J_{1}, J_{2}\right)}} \sum_{n_{1}} \frac{J_{1}^{n_{1} / 2}}{\sqrt{\rho_{1}\left(n_{1}\right)}} e^{-i \gamma_{1} e_{1}} \frac{1}{\sqrt{\mathcal{N}_{2}\left(n_{1}, J_{2}\right)}} \sum_{n_{2}} \frac{J_{2}^{n_{2} / 2}}{\sqrt{\rho_{2}\left(n_{1}, n_{2}\right)}} e^{-i \gamma_{1} e_{2}}\left|n_{1}, n_{2}\right\rangle
$$

Já definimos um tipo de estado coerente generalizado para tal espaço em (4.39). Apresentamos agora dois outros.

1) GKS-GKS: A escolha tradicional $\rho_{n_{i}}=n_{i}$ !, $e_{i}=n_{i}, i=1,2$ fornece $\mathrm{o}$ produto tensorial de dois estados GKS independentes:

$$
\left|z_{1}, z_{2}\right\rangle=e^{-\left(\left|z_{1}\right|^{2}+\left|z_{2}\right|^{2}\right) / 2} \sum_{n_{1}=0}^{\infty} \sum_{n_{2}=0}^{\infty} \frac{z_{1}^{n_{1}}}{\sqrt{n_{1} !}} \frac{z_{2}^{n_{2}}}{\sqrt{n_{2} !}}\left|n_{1}, n_{2}\right\rangle, \quad z_{j}=\sqrt{J_{j}} e^{-i \gamma_{j}}
$$

Esses estados são obviamente supercompletos e sua função peso é simplesmente $1 / \pi^{2}$

2) GKS-su(1,1): Nesse caso introduzimos variáveis de coerência para o primeiro grau de liberdade da seguinte forma:

$$
\left|n_{1}, J_{2}, \gamma_{2}\right\rangle=\left(1-J_{2}\right)^{\left(n_{1}+2\right) / 2} \sum_{n_{2}=0}^{\infty}\left(\begin{array}{c}
n_{1}+n_{2}+1 \\
n_{2}
\end{array}\right)^{\frac{1}{2}} J_{2}^{n_{2} / 2} e^{-i \gamma_{2} n_{2}}\left|n_{1}, n_{2}\right\rangle
$$


Neste passo temos, assim como em (4.20), a resolução do projetor

$$
\mathbf{1}_{n_{1}}=\sum_{n_{2}=0}^{\infty}\left|n_{1}, n_{2}\right\rangle\left\langle n_{1}, n_{2}\right|
$$

Agora introduzimos o segundo par de variáveis de coerência, novamente de uma forma "tipo GKS":

$$
\left|J_{1}, J_{2}, \gamma_{1}, \gamma_{2}\right\rangle=e^{-J_{1} / 2} \sum_{n_{1}=0}^{\infty} \frac{J_{1}^{n_{1} / 2} e^{-i \gamma_{1} n_{1}}}{\sqrt{n_{1} !}}\left|n_{1}, J_{2}, \gamma_{2}\right\rangle
$$

A resolução completa da identidade fica

$$
\mathbf{1}=\int d \mu\left(J_{1}, J_{2}, \gamma_{1}, \gamma_{2}\right)\left|J_{1}, J_{2}, \gamma_{1}, \gamma_{2}\right\rangle\left\langle J_{1}, J_{2}, \gamma_{1}, \gamma_{2}\right|
$$

onde

$$
\begin{aligned}
\int d \mu\left(J_{1}, J_{2}, \gamma_{1}, \gamma_{2}\right) & =\int_{0}^{2 \pi} d \gamma_{1} \int_{0}^{2 \pi} d \gamma_{2} \int_{0}^{\infty} d J_{1} \int_{0}^{1} d J_{2} W\left(J_{1}, J_{2}\right), \\
W\left(J_{1}, J_{2}\right) & =\frac{1}{4 \pi^{2}} \frac{J_{1}}{\left(1-J_{2}\right)^{2}}
\end{aligned}
$$

\subsection{Aplicação a magnetismo bidimensional}

A Hamiltoniana para elétrons sem spin confinados em duas dimensões por um potencial harmônico isotrópico e sujeitos a um campo magnético constante B pode ser escrita

$$
H=\frac{1}{2 m}\left(\mathbf{P}+\frac{e}{c} \mathbf{A}\right)^{2}+\frac{1}{2} m \omega_{0}^{2} \mathbf{R}^{2}
$$

onde as interações de Coulomb foram desprezadas. No gauge simétrico $\mathbf{A}=\frac{1}{2} \mathbf{B} \times$ $\mathbf{R}$ esta Hamiltoniana pode ser escrita como a soma de um oscilador harmônico e 
um operador de momentum angular

$$
H=\left[\left(\frac{1}{2 m} P_{x}^{2}+\frac{1}{8} m \omega^{2} X^{2}\right)+\left(\frac{1}{2 m} P_{y}^{2}+\frac{1}{8} m \omega^{2} Y^{2}\right)\right]+\frac{\omega_{c}}{2} L_{0} \equiv H_{0}+L_{z}
$$

onde $\omega_{c}=e B / m c$ é a frequência de ciclotron, $\omega=\sqrt{\omega_{c}^{2}+4 \omega_{0}^{2}}$ e $L_{0}=X P_{y}-Y P_{x}$.

Em vez de trabalhar diretamente com os operadores de criação e destruição,

$$
a_{x}=\frac{1}{\sqrt{2}}\left(\frac{X}{l_{0}}+\frac{i l_{0}}{\hbar} P_{x}\right), a_{y}=\frac{1}{\sqrt{2}}\left(\frac{Y}{l_{0}}+\frac{i l_{0}}{\hbar} P_{y}\right)
$$

(onde $l_{0}=\sqrt{2 \hbar / m \omega}$ ), podemos trabalhar com suas combinações lineares:

$$
a_{1}=\frac{1}{\sqrt{2}}\left(a_{x}-i a_{y}\right), \quad a_{2}=\frac{1}{\sqrt{2}}\left(a_{x}+i a_{y}\right) .
$$

Note que $a_{1}$ e $a_{2}$ são operadores bosônicos: $\left[a_{1}, a_{1}^{\dagger}\right]=\mathrm{I}=\left[a_{2}, a_{2}^{\dagger}\right]$. Os operadores $H_{0}$ e $L_{z}$ podem ser escritos em termos de operadores número $N_{1}=a_{1}^{\dagger} a_{1}$ e $N_{2}=$ $a_{2}^{\dagger} a_{2}$ como:

$$
H_{0}=\frac{\hbar \omega}{2}\left(N_{1}+N_{2}+1\right), \quad L_{z}=\frac{\hbar \omega_{c}}{2}\left(N_{1}-N_{2}\right) .
$$

Os autovetores da Hamiltoniana total são produtos tensoriais de estados de Fock:

$$
\left|n_{1}, n_{2}\right\rangle=\frac{1}{\sqrt{n_{1} ! n_{2} !}}\left(a_{1}^{\dagger}\right)^{n_{1}}\left(a_{2}^{\dagger}\right)^{n_{2}}|0,0\rangle
$$

No que se segue faremos uso de três famílias de estados coerentes generalizados apresentadas anteriormente para obter desigualdades de Berezin-Lieb para o potencial termodinâmico associado com a Hamiltoniana (4.60), conforme apresentado em [63]. Esse sistema foi considerado em [64], onde os autores derivaram um resultado analítico exato para o potencial termodinâmico. Nossa abordagem fornece apenas limites para essa quantidade, e recupera os resultados de [64] em alguns casos limite. 


\subsubsection{Simetria do oscilador harmônico}

Vamos primeiro utilizar os estados (4.52):

$$
\left|z_{1}, z_{2}\right\rangle=\exp \left[-\frac{1}{2}\left(\left|z_{1}\right|^{2}+\left|z_{2}\right|^{2}\right)\right] \sum_{n_{1}, n_{2}} \frac{z_{1}^{n_{1}}}{\sqrt{n_{1} !}} \frac{z_{2}^{n_{2}}}{\sqrt{n_{2} !}}\left|n_{1}, n_{2}\right\rangle
$$

Neste caso os símbolos maior e menor da Hamiltoniana total são

$$
2 \hat{H}=\hbar \omega\left(\left|z_{1}\right|^{2}+\left|z_{2}\right|^{2}-1\right)+\hbar \omega_{c}\left(\left|z_{1}\right|^{2}-\left|z_{2}\right|^{2}\right)=2 \check{H}-2 \hbar \omega .
$$

As desigualdades BL para o potencial termodinâmico $\Omega=-\frac{1}{\beta} \operatorname{Tr} \ln \left(1+e^{-\beta(H-\mu)}\right)$, onde $\mu$ é o potencial químico e $\beta=1 / k_{B} T$, são dadas por

$$
-\frac{1}{\beta \pi^{2}} \int \ln \left(1+e^{-\beta(\hat{H}-\mu)}\right) d^{2} z_{1} d^{2} z_{2} \leq \Omega \leq-\frac{1}{\beta \pi^{2}} \int \ln \left(1+e^{-\beta(\check{H}-\mu)}\right) d^{2} z_{1} d^{2} z_{2}
$$

Fazendo as substituições

$$
\begin{aligned}
& u=\hbar \beta\left[\left|z_{1}\right|^{2}\left(\omega+\omega_{c}\right)+\left|z_{2}\right|^{2}\left(\omega-\omega_{c}\right)\right] \\
& v=\hbar \beta\left|z_{1}\right|^{2}\left(\omega+\omega_{c}\right)
\end{aligned}
$$

elas se reduzem a

$$
-\frac{1}{\beta} \int_{0}^{\infty} d u \int_{0}^{u} d v \ln \left(1+\kappa_{+} e^{-u}\right) \leq \Omega \leq-\frac{1}{\beta} \int_{0}^{\infty} d u \int_{0}^{u} d v \ln \left(1+\kappa_{-} e^{-u}\right)
$$

e, depois de uma integração por partes, eventualmente se tornam

$$
\phi\left(\kappa_{+}\right) \leq \Omega \leq \phi\left(\kappa_{-}\right)
$$


onde $\kappa_{ \pm}=e^{\beta(\mu \pm \hbar \omega / 2)}$. A função $\phi$ é dada por

$$
\begin{aligned}
\phi(\kappa) & =-\frac{\kappa}{2 \beta\left(\beta \hbar \omega_{0}\right)^{2}} \int_{0}^{\infty} \frac{u^{2} e^{-u}}{1+\kappa e^{-u}} d u \\
& =\frac{1}{\beta\left(\beta \hbar \omega_{0}\right)^{2}} \begin{cases}F_{3}(-\kappa) & \text { para } \kappa \leq 1, \\
F_{3}\left(-\kappa^{-1}\right)-\frac{(\ln \kappa)^{3}}{6}-\frac{\pi^{2} \ln \kappa}{6}, & \text { para } \kappa>1,\end{cases}
\end{aligned}
$$

com

$$
F_{s}(z)=\sum_{m=1}^{\infty} \frac{z^{m}}{m^{s}}
$$

Podemos usar as desigualdades (4.71) para estudar regimes extremos. Para o potencial químico e temperatura muito maiores que $\hbar \omega / 2$, ou alternativamente em um regime semiclássico, temos $\kappa_{ \pm} \approx e^{\beta \mu}>1$ e as desigualdades restringem o potencial termodinâmico para o valor:

$$
\Omega \approx-\frac{\mu}{6}\left(\frac{\mu}{\hbar \omega_{0}}\right)^{2}\left[1+\pi^{2}\left(\frac{k_{B} T}{\mu}\right)^{2}-6\left(\frac{k_{B} T}{\mu}\right)^{3} F_{3}\left(-e^{-\frac{\mu}{k_{B} T}}\right)\right] .
$$

Para temperaturas extremamente altas, $k_{B} T \gg \mu$, temos $\kappa_{ \pm} \approx 1$ de forma que o potencial termodinâmico é aproximadamente igual a:

$$
\Omega \approx k_{B} T\left(\frac{k_{B} T}{\hbar \omega_{0}}\right)^{2} F_{3}(-1)
$$

Esses resultados estão em concordância com os resultados exatos apresentados em [64].

\subsubsection{Simetria $s u(2)$}

Essa simetria pode ser colocada em evidência introduzindo os operadores $L_{+}=$ $a_{1}^{\dagger} a_{2}$ e $L_{-}=a_{2}^{\dagger} a_{1}$. As relações de comutação são:

$$
\left[L_{+}, L_{-}\right]=2 \frac{L_{z}}{\hbar \omega_{c}},\left[\frac{L_{z}}{\hbar \omega_{c}}, L_{ \pm}\right]= \pm L_{ \pm}
$$


e o operador invariante de Casimir é dado por

$$
\mathcal{C}=\frac{1}{2}\left(L_{+} L_{-}+L_{-} L_{+}\right)+\left(\frac{L_{z}}{\hbar \omega_{c}}\right)^{2}=\left(\frac{N_{1}+N_{2}}{2}\right)\left(\frac{N_{1}+N_{2}}{2}+1\right) .
$$

Portanto, para um valor fixo $j=\left(n_{1}+n_{2}\right) / 2$ do operador $\left(N_{1}+N_{2}\right) / 2=$ $H_{0} / \hbar \omega-1 / 2$, existe uma representação $(2 j+1)$-dimensional de $s u(2)$ na qual o operador $L_{z} /\left(\hbar \omega_{c}\right)$ tem seu espectro no intervalo $-j \leq m=\left(n_{1}-n_{2}\right) / 2 \leq j$. Note que no limite de campo fraco $\omega_{c}<<\omega_{0}$ os níveis de energia $E_{n_{1}, n_{2}}=$ $\frac{\hbar \omega}{2}\left(n_{1}+n_{2}+1\right)+\frac{\hbar \omega_{c}}{2}\left(n_{1}-n_{2}\right)$ podem ser aproximados por $E_{j}=\hbar \omega_{0}(2 j+1)$.

Essa simetria sugere o uso dos estados (4.39)

$|J, \gamma, \theta, \varphi\rangle=e^{-J / 2} \sum_{j \in \mathbb{N} / 2} \frac{J^{j}}{\sqrt{(2 j) !}} e^{-i 2 j \gamma} \sum_{m=-j}^{j}\left(\begin{array}{c}2 j \\ j+m\end{array}\right)^{\frac{1}{2}}\left(\cos \frac{\theta}{2}\right)^{j+m}\left(\sin \frac{\theta}{2}\right)^{j-m} e^{-i(j+m) \varphi}|j, m\rangle$,

que são supercompletos com medida dada por

$$
\int d \mu(J, \gamma, \theta, \varphi)=\int_{0}^{2 \pi} d \gamma \int_{0}^{\pi} \sin \theta d \theta \int_{0}^{2 \pi} d \varphi \int_{0}^{\infty} d J \frac{J}{8 \pi^{2}}
$$

As relações

$$
\begin{gathered}
H_{0}-\frac{\hbar \omega}{2}=\hbar \omega \int d \mu(J, \gamma, \theta, \varphi) \frac{J-2}{2}|J, \gamma, \theta, \varphi\rangle\langle J, \gamma, \theta, \varphi|, \\
L_{z}=\hbar \omega_{c} \int d \mu(J, \gamma, \theta, \varphi) \frac{J}{2} \cos \theta|J, \gamma, \theta, \varphi\rangle\langle J, \gamma, \theta, \varphi|, \\
\left\langle J, \gamma, \theta, \varphi\left|H_{0}\right| J, \gamma, \theta, \varphi\right\rangle=\frac{\hbar \omega}{2}(J+1), \\
\left\langle J, \gamma, \theta, \varphi\left|L_{z}\right| J, \gamma, \theta, \varphi\right\rangle=\hbar \omega_{c} \frac{J}{2} \cos \theta
\end{gathered}
$$

nos permitem escrever os símbolos maior e menor da Hamiltoniana total:

$$
\check{H}=\frac{J}{2}\left(\hbar \omega+\hbar \omega_{c} \cos \theta\right)+\frac{\hbar \omega}{2}=\hat{H}+\hbar \omega .
$$


As desigualdades BL

$$
\frac{-1}{\beta} \int d \mu(J, \gamma, \theta, \varphi) \ln \left[1+e^{-\beta(\hat{H}-\mu)}\right] \leq \Omega \leq \frac{-1}{\beta} \int d \mu(J, \gamma, \theta, \varphi) \ln \left[1+e^{-\beta(\check{H}-\mu)}\right]
$$

neste caso envolvem a integral

$$
\int d S \int_{0}^{\infty} d J J \ln \left[1+\kappa_{ \pm} e^{-\frac{\beta}{2}\left(\hbar \omega+\hbar \omega_{c} \cos \theta\right) J}\right]=2 \pi \int_{-1}^{1} d y \int_{0}^{\infty} d J J \ln \left[1+\kappa_{ \pm} e^{-\frac{\beta}{2}\left(\hbar \omega+\hbar \omega_{c} y\right) J}\right],
$$

onde novamente $\kappa_{ \pm}=e^{\beta(\mu \pm \hbar \omega / 2)}$ e onde fizemos a substituição $y=\cos \theta$. Portanto, uma vez que

$$
\int_{0}^{\infty} x \ln \left(1+k e^{-c x}\right) d x=\frac{-1}{c^{2}} \begin{cases}F_{3}(-k) & \text { para } k \leq 1, c>0 \\ F_{3}\left(-k^{-1}\right)-\frac{(\ln k)^{3}}{6}-\frac{\pi^{2} \ln k}{6}, & \text { para } k>1, c>0\end{cases}
$$

e

$$
\int_{-1}^{1} \frac{d y}{\left(\omega+\omega_{c} y\right)^{2}}=\frac{2}{\left(\omega^{2}-\omega_{c}^{2}\right)}=\frac{1}{2 \omega_{0}^{2}}
$$

podemos escrever (4.86) novamente como

$$
\phi\left(\kappa_{+}\right) \leq \Omega \leq \phi\left(\kappa_{-}\right)
$$

onde $\phi(\kappa)$ é dado por (4.72).

O fato de havermos obtido o mesmo resultado usando ambas as famílias de estados coerente generalizados não é devido a nenhuma particularidade da Hamiltoniana. De fato, as integrais em (4.67) e (4.87) estão relacionadas através da mudança de variáveis

$$
\left|z_{1}\right|^{2}+\left|z_{2}\right|^{2}=J, \quad\left|z_{1}\right|^{2}-\left|z_{2}\right|^{2}=J y
$$

e portanto serão iguais para qualquer Hamiltoniana (e não apenas para o potencial 
termodinâmico).

\subsubsection{Simetria $s u(1,1)$}

A estrutura $s u(1,1)$ que subjaz a esse modelo de magnetismo bidimensional pode ser exibida introduzindo-se os operadores:

$$
K_{+}=a_{1}^{\dagger} a_{2}^{\dagger}, \quad K_{-}=a_{1} a_{2}, \quad K_{0}=\frac{1}{2}\left(N_{1}+N_{2}+1\right)=\frac{H_{0}}{\hbar \omega} .
$$

É fácil ver que esses operadores satisfazem as relações de comutação de $s u(1,1)$. Portanto, o operador de Casimir é:

$\mathcal{D}=K_{0}^{2}-\frac{1}{2}\left(K_{+} K_{-}+K_{-} K_{+}\right)=\left(\frac{N_{1}-N_{2}}{2}+\frac{1}{2}\right)\left(\frac{N_{1}-N_{2}}{2}-\frac{1}{2}\right)=\left(\frac{L_{z}}{\hbar \omega_{c}}+\frac{1}{2}\right)\left(\frac{L_{z}}{\hbar \omega_{c}}-\frac{1}{2}\right)$

Quando $n_{1} \geq n_{2}$, para um valor fixo $\eta=\left(n_{1}-n_{2}+1\right) / 2 \geq 1 / 2$ do operador $\left(N_{1}-N_{2}+1\right) / 2$, existe uma representação de $s u(1,1)$ na série discreta, na qual o operador $K_{0}$ tem seu espectro no intervalo infinito $\eta, \eta+1, \eta+2, \ldots$ Alternativamente, quando $n_{1} \leq n_{2}$, para um valor fixo $\rho=\left(-n_{1}+n_{2}+1\right) / 2 \geq 1 / 2$ do operador $\left(-N_{1}+N_{2}+1\right) / 2$, existe uma representação de $s u(1,1)$ na qual o operador $K_{0}$ tem seu espectro no intervalo infinito $\rho, \rho+1, \rho+2, \ldots$.

Essa simetria é especialmente adaptada para o limite de campos intensos, no qual $\omega_{c} \gg \omega_{0}$ e os níveis de energia podem ser aproximados por $E_{n_{1}, n_{2}} \approx$ $\hbar \omega_{c}\left(n_{1}+1 / 2\right)$. Portanto, para o dado valor de $n_{1}$, que corresponde ao índice do nível de Landau, temos uma degenerescência infinita etiquetada por $n_{2}$. Podemos reinterpretar esse fato em termos da simetria $s u(1,1)$ notando que, para um dado valor de $\left(n_{1}-n_{2}\right)$, os estados estacionários são pesos de alguma série discreta dessa álgebra.

A simetria $s u(1,1)$ deve ser explorada de maneira diferente da simetria $s u(2)$ anterior. Primeiro, temos que decompor nosso espaço de Hilbert na soma direta 
de três subespaços ou setores, que correspondem a $n_{1}>n_{2}, n_{1}=n_{2}$ e $n_{1}<n_{2}$ : $\mathcal{H}=\mathcal{H}_{>} \oplus \mathcal{H}_{=} \oplus \mathcal{H}_{<}$. Da mesma forma, o traço de uma função $g$ da Hamiltoniana se decompõe como: $\operatorname{Tr}(g(H))=T r_{>}(g(H))+\operatorname{Tr}_{=}(g(H))+T r_{<}(g(H))$. Podemos então aplicar as desigualdades BL em cada setor.

No primeiro setor usamos como estados coerentes a superposição (4.55):

$\left|J_{1}, J_{2}, \gamma_{1}, \gamma_{2}\right\rangle=e^{-J_{1} / 2} \sum_{n=0}^{\infty} \frac{J_{1}^{n / 2}}{\sqrt{n !}} e^{-i n \gamma_{1}}\left(1-J_{2}\right)^{(n+2) / 2} \sum_{m=0}^{\infty}\left(\begin{array}{c}n+m+1 \\ m\end{array}\right)^{\frac{1}{2}} J_{2}^{m / 2} e^{-i m \gamma_{2}}|n, m\rangle$,

onde $n=n_{1}-n_{2}-1, m=n_{2}$.

Para o setor $n_{1}=n_{2}=n$ nós associamos simplesmente os estados GKS tradicionais

$$
\left|J_{3}, \gamma_{3}\right\rangle=e^{-J_{3} / 2} \sum_{n=0}^{\infty} \frac{J_{3}^{n / 2}}{\sqrt{n !}} e^{-i n \gamma_{3}}|n\rangle .
$$

Ao último setor associamos estados análogos a (4.94) mas com $n=n_{2}-n_{1}-1, m=$ $n_{1}$.

Vamos nos concentrar no primeiro setor. A resolução do projetor

$$
\int d \mu\left(J_{1}, J_{2}, \gamma_{1}, \gamma_{2}\right)\left|J_{1}, J_{2}, \gamma_{1}, \gamma_{2}\right\rangle\left\langle J_{1}, J_{2}, \gamma_{1}, \gamma_{2}\left|=\sum_{n_{2}=0}^{\infty} \sum_{n_{1}>n_{2}}\right| n_{1}, n_{2}\right\rangle\left\langle n_{1}, n_{2}\right| \equiv \mathbf{1}_{>}
$$

vale com $\int d \mu\left(J_{1}, J_{2}, \gamma_{1}, \gamma_{2}\right)$ dada por (4.57)-(4.58). As restrições $H_{0>}=\mathbf{1}_{>} H_{0} \mathbf{1}_{>}$ e $L_{z>}=\mathbf{1}_{>} L_{z} \mathbf{1}_{>}$dos operadores $H_{0}$ e $L_{z}$ podem ser escritas:

$$
\begin{gathered}
H_{0>}=\hbar \omega \int d \mu\left(J_{1}, J_{2}, \gamma_{1}, \gamma_{2}\right) \frac{\left(J_{1}-2\right)}{2}\left(\frac{1+J_{2}}{1-J_{2}}\right)\left|J_{1}, J_{2}, \gamma_{1}, \gamma_{2}\right\rangle\left\langle J_{1}, J_{2}, \gamma_{1}, \gamma_{2}\right| \\
L_{z>}=\hbar \omega_{c} \int d \mu\left(J_{1}, J_{2}, \gamma_{1}, \gamma_{2}\right) \frac{\left(J_{1}-1\right)}{2}\left|J_{1}, J_{2}, \gamma_{1}, \gamma_{2}\right\rangle\left\langle J_{1}, J_{2}, \gamma_{1}, \gamma_{2}\right|
\end{gathered}
$$


e seus símbolos menores são dados por:

$$
\begin{aligned}
\check{H}_{0>} & =\hbar \omega \frac{\left(J_{1}+2\right)}{2}\left(\frac{1+J_{2}}{1-J_{2}}\right), \\
\check{L}_{z>} & =\hbar \omega_{c} \frac{\left(J_{1}+1\right)}{2} .
\end{aligned}
$$

Portanto os símbolos maiores e menores da restrição da Hamiltoniana total são:

$$
\begin{aligned}
& 2 \hat{H}_{>}=\hbar \omega\left(J_{1}-2\right)\left(\frac{1+J_{2}}{1-J_{2}}\right)+\hbar \omega_{c}\left(J_{1}-1\right), \\
& 2 \check{H}_{>}=\hbar \omega\left(J_{1}+2\right)\left(\frac{1+J_{2}}{1-J_{2}}\right)+\hbar \omega_{c}\left(J_{1}+1\right) .
\end{aligned}
$$

A integral do limite inferior nas desigualdades BL restritas ao primeiro setor são dadas por:

$-\frac{1}{\beta} \int d \mu\left(J_{1}, J_{2}, \gamma_{1}, \gamma_{2}\right) \ln \left[1+e^{-\beta\left(\hat{H}_{>}-\mu\right)}\right]=-\frac{1}{\beta} \int_{0}^{\infty} d J \int_{1}^{\infty} d y \frac{J}{2} \ln \left[1+\sigma_{+}(y) e^{-\frac{\beta}{2}\left(\hbar \omega y+\hbar \omega_{c}\right) J}\right]$

(com a substituição $\left.y=\frac{1+J_{2}}{1-J_{2}}\right)$, onde

$$
\sigma_{ \pm}(y)=e^{ \pm \beta\left(\hbar \omega y+\hbar \omega_{c} / 2 \pm \mu\right)}
$$

Uma vez que $\sigma_{+}(y)$ é sempre maior que 1 , temos para (4.103)

$$
\frac{2}{\beta^{3} \hbar^{2}} \int_{1}^{\infty} \frac{d y}{\left(\omega y+\omega_{c}\right)^{2}}\left\{F_{3}\left(-\sigma_{+}(y)^{-1}\right)-\frac{\left(\ln \sigma_{+}(y)\right)^{3}}{6}-\frac{\pi^{2} \ln \sigma_{+}(y)}{6}\right\}
$$

Essa integral é divergente e portanto não fornece limite inferior para o potencial termodinâmico. Para a integral associada ao limite superior temos

$$
-\frac{1}{\beta} \int_{0}^{\infty} d J \int_{1}^{\infty} d y \frac{J}{2} \ln \left[1+\sigma_{-}(y) e^{-\frac{\beta}{2}\left(\hbar \omega y+\hbar \omega_{c}\right) J}\right]
$$


Quando $\sigma_{-}(y)$ é maior que 1 a integral diverge. Portanto vamos assumir $\hbar \omega+$ $\hbar \omega_{c} / 2>\mu$ e então (4.106) se torna

$-\frac{2}{\beta^{3} \hbar^{2}} \int_{1}^{\infty} d y \frac{F_{3}\left(-\sigma_{-}(y)\right)}{\left(\omega y+\omega_{c}\right)^{2}}=-\frac{2}{\beta^{3} \hbar^{2}} \sum_{n=1}^{\infty} \frac{(-1)^{n} e^{-n \beta\left(\hbar \omega_{c} / 2-\mu\right)}}{n^{3} \omega\left(\omega+\omega_{c}\right)} E_{2}\left(n \beta \hbar\left(\omega+\omega_{c}()\right), 107\right)$

onde

$$
E_{n}(x)=\int_{1}^{\infty} \frac{e^{-x t}}{t^{n}} d t
$$

Para o setor definido por $n_{1}=n_{2}=n$ temos

$$
\begin{aligned}
\sum_{n=0}^{\infty}|n\rangle\langle n| & =\frac{1}{2 \pi} \int_{0}^{2 \pi} d \gamma \int_{0}^{\infty} d J|J, \gamma\rangle\langle J, \gamma|, \\
\check{H}_{0=} & =\frac{\hbar \omega}{2}(2 J+1)=\hat{H}_{0=}+\hbar \omega .
\end{aligned}
$$

Os limites sobre o espaço de Hilbert total são obtidos somando-se os resultados para cada setor. Finalmente obtemos a desigualdade:

$$
\Omega<\psi\left(e^{-\beta(\hbar \omega / 2-\mu)}\right)-\frac{2}{\beta^{3} \hbar^{2}} \sum_{n=1}^{\infty} \frac{(-1)^{n} e^{-n \beta\left(\hbar \omega_{c} / 2-\mu\right)}}{n^{3} \omega\left(\omega+\omega_{c}\right)} E_{2}\left(n \beta \hbar\left(\omega+\omega_{c}\right)\right)
$$

onde $\psi(\kappa)$ é dado por

$$
\begin{aligned}
\psi(\kappa) & =-\frac{1}{2 \pi \beta} \int_{0}^{2 \pi} d \gamma \int_{0}^{\infty} \ln \left[1+\kappa e^{-\beta \hbar \omega J}\right] d J \\
& =-\frac{1}{\beta^{2} \hbar \omega} \begin{cases}-F_{2}(-\kappa) & \text { para } \kappa<1 \\
F_{2}\left(-\kappa^{-1}\right)+\frac{1}{2}(\ln \kappa)^{2}-2 F_{2}(-1) & \text { caso contrário. }\end{cases}
\end{aligned}
$$

A desigualdade (4.111) é bastante diferente de (4.71), mostrando que em geral os resultados obtidos usando famílias diferentes de estados coerentes não serão os mesmos. Em particular, nenhum limite inferior pode ser obtido usando os estados coerentes de $s u(1,1)$ devido à divergência da integral (4.105).

Os três diferentes tipos de estados coerentes que foram usados neste modelo 


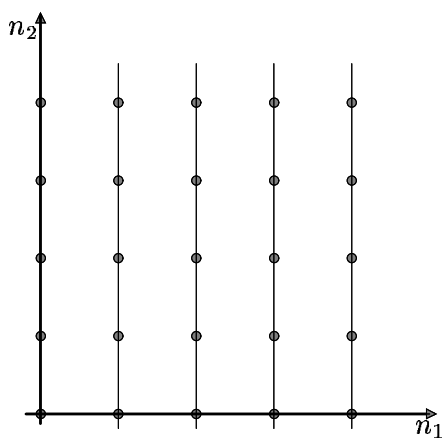

(a)

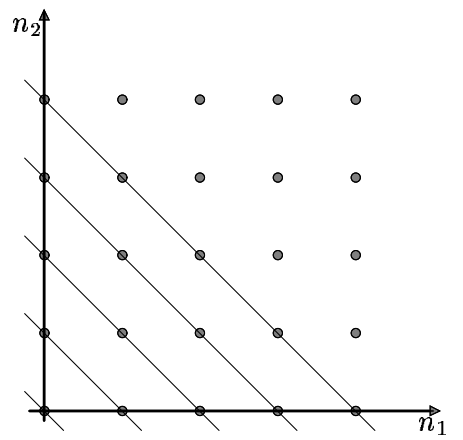

(b)

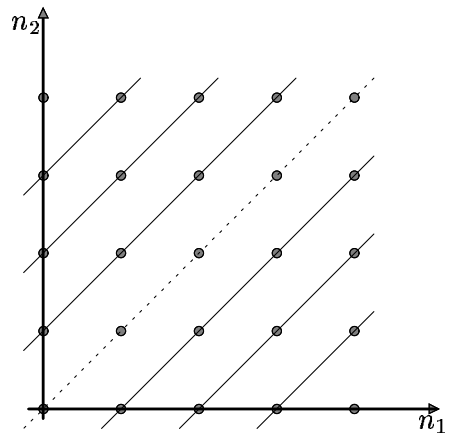

(c)

Figura 4.1: Representação pictórica do espaço de estados $\left|n_{1}, n_{2}\right\rangle$. (a) Representação irredutíveis do oscilador harmônico. (b) Representações irredutíveis de $s u(2)$. (c) A série discreta de $s u(1,1)$; linhas acima (abaixo) da linha pontilhada pertencem ao primeiro (segundo) setor definido no texto

bidimensional têm uma visualização geométrica simples, mostrada na figura 4.1. Em cada subfigura apresentamos uma perspectiva diferente do espaço de Hilbert de estados do sistema (a cada ponto corresponde um estado $\left|n_{1}, n_{2}\right\rangle$ ), e as representaọes de $s u(2)$ e $s u(1,1)$ envolvidas.

\subsection{Poço de mínimos duplos}

Nesta seção construiremos estados GK para o poço do mínimos duplos unidimensional, um sistema que não possui estrutura algébrica e portanto não é adequado para a definição de estados coerentes de Perelomov [6]. Os resultados apresentados aqui apareceram em [65].

\subsubsection{O potencial de mínimos duplos clássico}

Seja

$$
\varepsilon=\frac{1}{2}\left(\frac{d x}{d t}\right)^{2}-\frac{\beta}{2} x^{2}+x^{4}
$$

a energia do sistema em unidades adimensionais. Note que este potencial possui uma barreira cuja altura depende de $\beta$ (veja figura 4.2) e que ao valor $\beta=0$ cor- 
responde o potencial quártico. Valores negativos de $\varepsilon$ correspondem a movimento "confinado" - abaixo da barreira. Podemos reescrever (4.114) como

$$
\frac{d x}{d t}=\sqrt{2\left[\lambda^{2}-\left(x^{2}-x_{0}^{2}\right)^{2}\right]}
$$

onde

$$
x_{0}^{2}=\frac{\beta}{4}, \quad \lambda=|\sqrt{\varepsilon+U}|, \quad U=\frac{\beta^{2}}{16} .
$$

Note que $U$ é a altura da barreira e que $x_{0}$ são os pontos de mínimo do potencial. Os pontos de retorno são $\pm \sqrt{x_{0}^{2} \pm \lambda}$.

Se $\varepsilon>0$ podemos definir

$$
a^{2}=\lambda-x_{0}^{2}, \quad b^{2}=\lambda+x_{0}^{2}
$$

e integrar por quadratura

$$
t+t_{0}=\int_{0}^{x} \frac{d y}{\sqrt{2\left(a^{2}+y^{2}\right)\left(b^{2}-y^{2}\right)}}=\frac{1}{\sqrt{2\left(a^{2}+b^{2}\right)}} s d^{-1}\left(\frac{x \sqrt{\left(a^{2}+b^{2}\right)}}{a b}, \sqrt{\frac{b^{2}}{a^{2}+b^{2}}}\right)
$$

para obter a solução

$$
x(t)=\sqrt{\frac{\varepsilon}{2 \lambda}} s d\left(2 \sqrt{\lambda}\left(t+t_{0}\right), \sqrt{\frac{\lambda+x_{0}^{2}}{2 \lambda}}\right),
$$

onde $s d$ é uma função de Jacobi.

Uma vez que a integral elíptica completa de primeiro tipo

$$
K(\kappa)=\int_{0}^{1} \frac{d t}{\sqrt{\left(1-t^{2}\right)\left(1-\kappa^{2} t^{2}\right)}}
$$

é um quarto do período da função de Jacobi $s d(u, \kappa)$, temos o período do movi- 
mento como função da energia:

$$
T_{\varepsilon>0}=\frac{2}{\sqrt{\lambda}} K(\kappa)
$$

onde

$$
\kappa=\sqrt{\frac{\lambda+x_{0}^{2}}{2 \lambda}}
$$

A relação entre a variável de ação

$$
S_{\varepsilon>0}=4 \int_{0}^{T / 4}\left(\frac{d x}{d t}\right)^{2} d t=\frac{4 \epsilon}{\sqrt{\lambda}} \int_{0}^{K(\kappa)} c d^{2}(u, \kappa) n d^{2}(u, \kappa) d u
$$

e a energia pode ser obtida [66]

$$
S_{\varepsilon>0}=\frac{8}{3} \sqrt{\lambda}\left[\left(\lambda-x_{0}^{2}\right) K(\kappa)+2 x_{0}^{2} E(\kappa)\right]
$$

onde

$$
E(\kappa)=\int_{0}^{1} \sqrt{d t \frac{1-\kappa^{2} t^{2}}{1-t^{2}}}
$$

é a integral elíptica completa de segundo tipo.

Na região confinada $\varepsilon<0$ um cálculo análogo resulta em

$$
x(t)=\sqrt{x_{0}^{2}-\lambda} n d\left(\sqrt{2} \sqrt{\lambda+x_{0}^{2}}\left(t+t_{0}\right), k^{-1}\right),
$$

e neste caso o período do movimento e a variável de ação são, respectivamente,

$$
\begin{aligned}
T_{\varepsilon<0} & =\sqrt{\frac{2}{\lambda+x_{0}^{2}}} K\left(\kappa^{-1}\right), \\
S_{\varepsilon<0} & =\frac{8}{3 \sqrt{2}} \sqrt{\lambda+x_{0}^{2}}\left[\left(\lambda-x_{0}^{2}\right) K\left(\kappa^{-1}\right)+x_{0}^{2} E\left(\kappa^{-1}\right)\right] .
\end{aligned}
$$

Esse sistema possui comportamento singular para $\varepsilon=0$, no qual a trajetória é chamada separatrix, quando o período tende a infinito e a variável e ação possui 
uma descontinuidade. Nesse caso temos a integral de quadratura (assumindo que a partícula esteja no ponto de retorno em $t=0$ )

$$
t=\int_{\sqrt{\beta / 2}}^{x} \frac{d y}{\sqrt{2 y^{2}\left(\beta / 2-y^{2}\right)}}=\frac{1}{\sqrt{\beta}} \ln \left[\frac{\sqrt{\beta}+\sqrt{\beta+2 x^{2}}}{\sqrt{2} x}\right]
$$

que resulta

$$
x(t)=\sqrt{\frac{\beta}{2}} \frac{1}{\cosh (\sqrt{\beta} t)} .
$$

\subsubsection{O potencial de mínimos duplos quântico}

A equação de Schrödinger estacionária para este sistema é, em unidades adimensionais,

$$
\left(\frac{1}{2} \frac{d^{2}}{d x^{2}}-\frac{\beta}{2} x^{2}+x^{4}\right) \phi_{n}(x)=e_{n} \phi_{n}(x)
$$

A altura da barreira de potencial é $U=\beta^{2} / 16$. Apesar da solução clássica exposta acima ser exata, a versão quântica não possui solução exata, e para obtermos os níveis de energia e os estados estacionários escrevemos a matriz Hamiltoniana na base de Fourier e a diagonalizamos numericamente, usando tipicamente algumas centenas de elementos.

Na figura 4.2 vemos os primeiros quatro níveis de energia (o potencial foi deslocado para que $e_{0}=0$ ) e as correspondentes autofunções, tanto para o potencial quártico, $\beta=0$, quanto para $\beta=10$. Note que no segundo caso o estado fundamental e o primeiro estado excitado são quase degenerados. Para altos valores de $\beta$ os estados abaixo da barreira tendem a aparecer em dubletos, e os correspondentes estados vêm em pares par-ímpar. Isso já pode ser observado na figura $4.2(\mathrm{~b})$.

Vamos agora considerar os estados definidos em (4.3) e investigar suas propriedades físicas. Uma vez que os níveis de energia $e_{n}$, neste caso, crescem mais rapidamente que $n$, a série (4.4) é convergente para todo valor de $J$. O cor- 


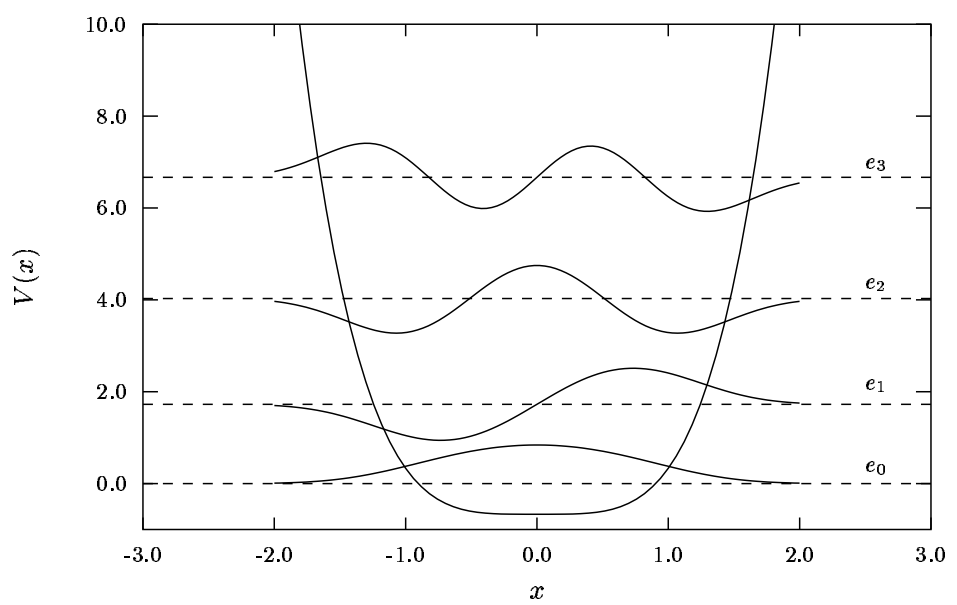

(a) $\beta=0$

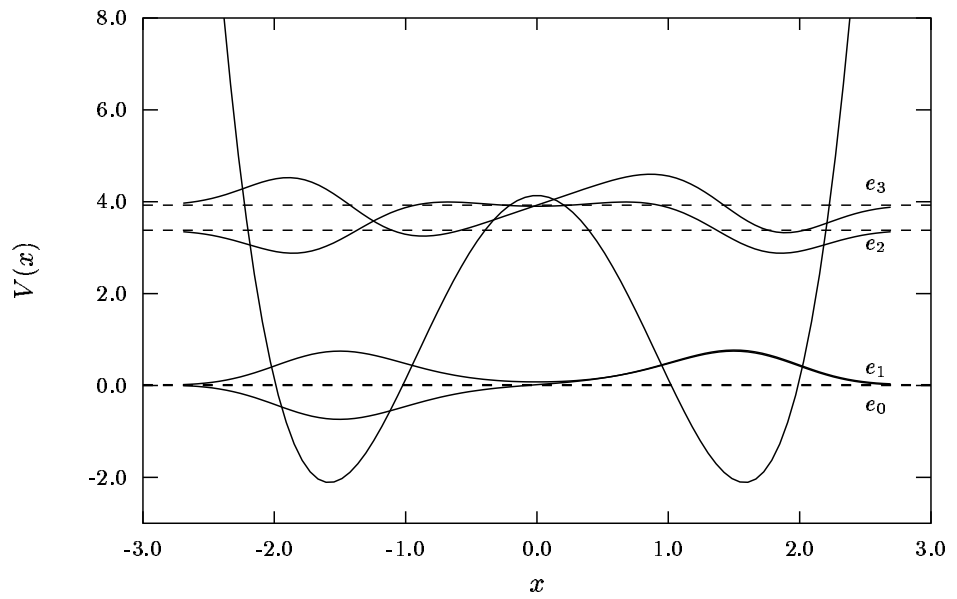

(b) $\beta=10$

Figura 4.2: Níveis de energia e estados estacionários para (a) o poço quártico e (b) o poço duplo $\operatorname{com} \beta=10$.

respondente problema de momentos (4.6), entretanto, não pode ser facilmente resolvido e, portanto, a completeza dessa família é ainda uma conjectura. Como $J$ corresponde à energia medida a partir do ponto zero, para $\beta>0$ haverá um valor crítico $J_{c}$, igual à altura da barreira, $J_{c}=U-e_{0}$, onde o sistema clássico é singular. 


\subsubsection{Estados coerentes generalizados}

A figura 4.3 mostra a densidade de probabilidade $|\langle x \mid J, \gamma\rangle|^{2}$ para um estados GK $\operatorname{com} \beta=20$, que resulta $J_{c} \simeq 21.9$. Usamos $J=25$, que é próximo à barreira de potencial. figuras $4.3(\mathrm{a})$ e $4.3(\mathrm{~b})$ correspondem a $t=0$ e $t=T / 4$, onde $T$ é o período clássico de uma partícula com energia $J-e_{0}$. Vemos que um pico inicialmente localizado evolui para uma distribuição intrincada, em contraste com os estados coerentes canônicos, refletindo a anharmonicidade de potencial. A fim de explorar as propriedades físicas desses estados, analisamos quantidades como sua natureza estatística, evolução temporal de $\Delta \hat{X} \Delta \hat{P}$, função de Wigner e autocorrelação.

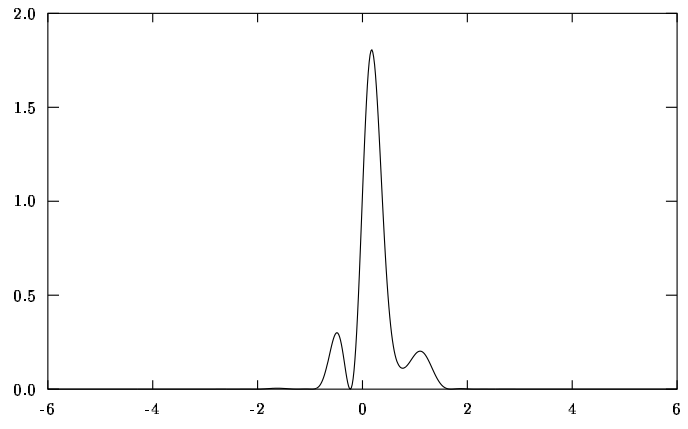

(a) $t=0$

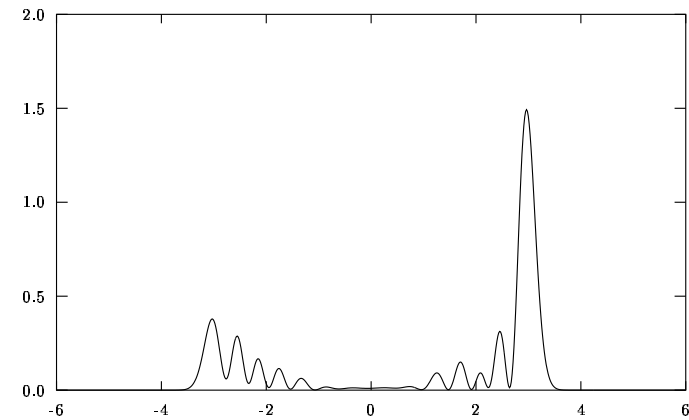

(b) $t=T / 4$

Figura 4.3: Densidade de probabilidade $|\langle x \mid J, \gamma\rangle|^{2}$ para estados GK em (a) $t=0$ e (b) $t=T / 4$ onde $T$ é o período clássico.

Começamos considerando o parâmetro de Mandel, figura 4.4, e notamos que os estados GK podem exibir todos os possíveis comportamentos. Vemos que $Q(J)$ é sempre negativo para pequenos valores de $J$, e que $Q(0)=0$ para qualquer $\beta$, de acordo com (4.12). De fato, quando $\beta>0$ os estados de baixa energia tendem a formar dubletos e a energia do estado excitado fica extremamente pequena. Nesse caso a equação (4.12) pode ser mais aproximada por $Q(J)=-J / e_{1}$. Como resultado, o parâmetro de Mandel vai de 0 a -1 em um intervalo extremamente pequeno de $J$, como vemos na figura 4.4(b). 


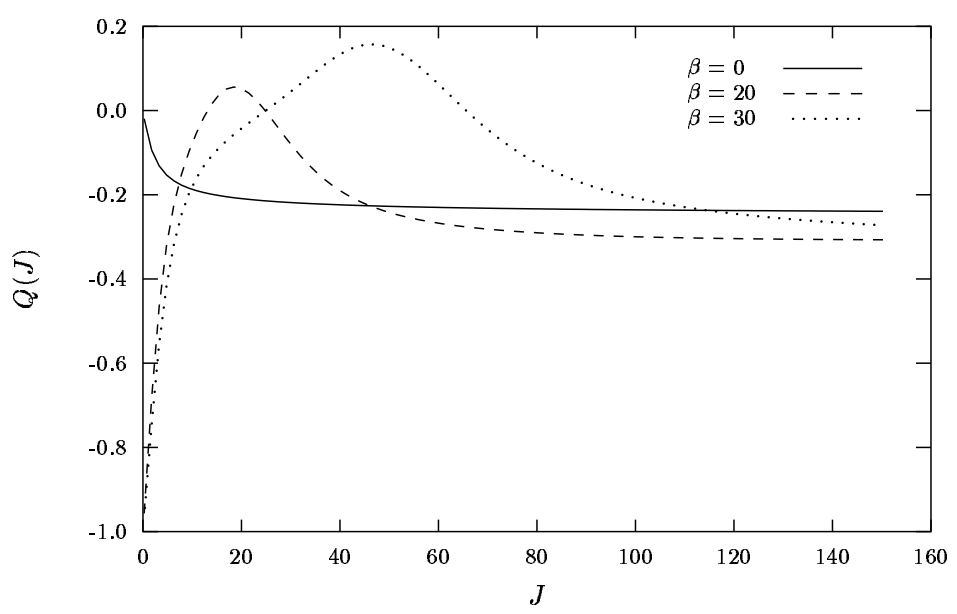

(a)

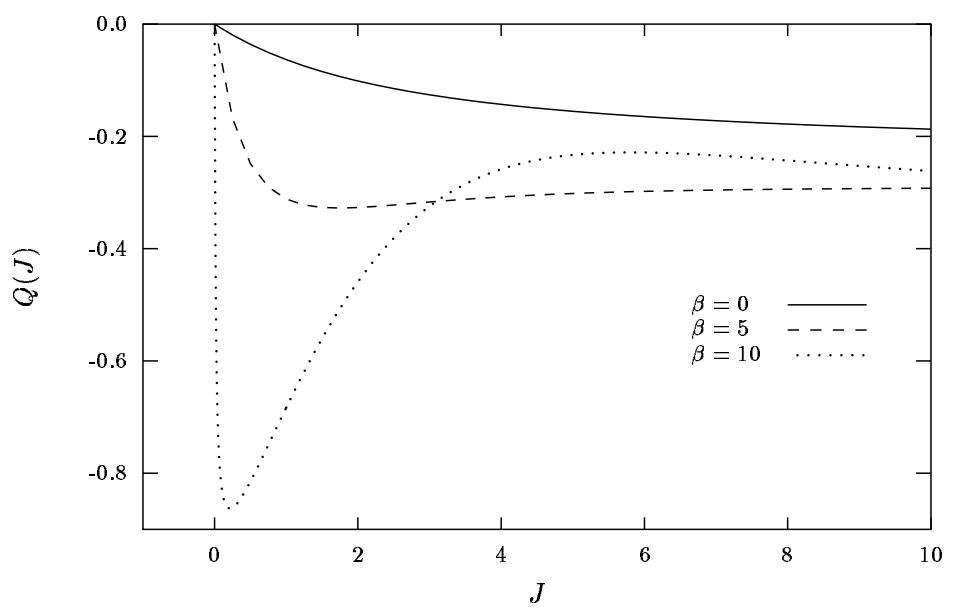

(b)

Figura 4.4: O parâmetro de Mandel pode ser negativo, zero ou positivo, dependendo de $\beta$ e $J$. Em (b) vemos apenas a região de $J$ pequeno.

Além disso, observamos que para grandes valores de $\beta$ os estados GK com $J=e_{1}$ envolvem essencialmente apenas os dois primeiros autoestados e podem ser proximados por

$$
\left|J=e_{1}, \gamma\right\rangle \simeq \frac{|0\rangle+e^{i e_{1} \gamma}|1\rangle}{\sqrt{2}}
$$

que para $\gamma=0$ é um estado localizado de um lado do poço. Conforme $\gamma$ evolui para $\pi / e_{1}$ o estado passa a ser $(|0\rangle-|1\rangle) / \sqrt{2}$, que está localizado do lado oposto. Portanto a partícula tunela de uma lado para o outro com uma frequência muito pequena, proporcional a $e_{1}$. Conforme $J$ cresce, outros estados de base se tornam 
importantes para a superposição de GK, e esse comportamento simples desaparece. Note que o parâmetro de Mandel para o estado (4.132) pode ser obtido analiticamente e é igual a -1 .

Os estados de Gazeau-Klauder para o potencial de mínimos duplos é portanto subpoissoniano para todo $J$ se $\beta$ for menor que um valor crítico $\beta_{c}$, um indicativo de sua natureza não-clássica. Quando $\beta>\beta_{c}$, por outro lado, eles podem se tornar super-poissonianos para todo um intervalo de $J$, como está claro a partir da figura 4.4(a). Essa flexibilidade está em contraste com o caso do oscilador harmônico, onde o estado coerente é sempre poissoniano (enquanto que os estados comprimidos [67] podem exibir todos os tipos de estatística).

Outra quantidade física de interesse é a dependência temporal da dispersão $\Delta \hat{X} \Delta \hat{P}$, que deve ser sempre maior ou igual a $\hbar / 2$ e que tem exatamente esse valor para o estado coerente canônico (4.1). Estados de mínima incerteza para potenciais gerais foram estudados em uma série de trabalhos [34], onde os autores discutem as condições para essa propriedade ser realizada. Uma análise do poço de mínimos duplos sob essa linha foi apresentada recentemente [68]. Estados coerentes derivados de álgebras de Lie também saturam uma relação de incerteza generalizada sob certas circunstâncias [1].

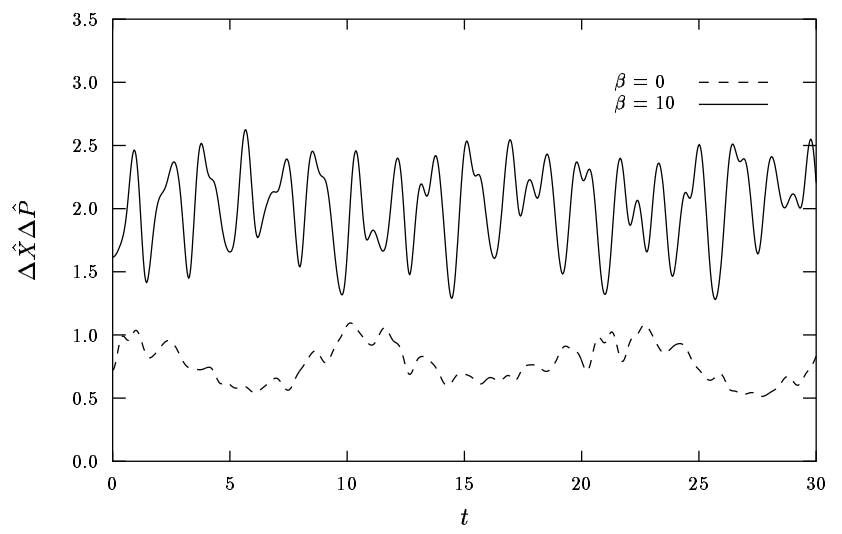

Figura 4.5: A dispersão $\Delta \hat{X} \Delta \hat{P}$ (em unidades de $\hbar$ ) para $J=1$ como função do tempo. Os estados GK não são de mínima incerteza.

Podemos ver na figura 4.5 a dispersão para os estados GK com $J=1$, medida 
em unidades de $\hbar$, tanto para $\beta=0$ quanto para $\beta=10$. Embora no primeiro caso a dispersão se aproxime marcadamente do valor mínimo $\frac{1}{2}$, vemos que os estados GK não são estados de mínima incerteza. Isso não nos surpreende, já que o potencial de mínimos duplos não possuem formulação algébrica. Note também que conforme $\beta$ aumenta também o faz a dispersão média, e os estados GK se tornam mais e mais diferentes de estados de mínima incerteza.

No apêndice A apresentamos uma análise do potencial de mínimos duplos em termos de distribuições de quase-probabilidade, as funções de Wigner e de Husimi, mas apenas os estados estacionários são considerados ali. Na figura 4.6 vemos as funções de Wigner $W(x, p)$ associadas com os estados GK com $J=1, \gamma=0$, novamente para $\beta=0$ e $\beta=10$. Vemos que essa função pode exibir uma estrutura razoavelmente complicada - além de ser par em $p$, não possui simetrias -, e é negativa em algumas porções do espaço de fase, indicando a natureza nãoclássica do estado correspondente. A função de Wigner não está localizada ao longo de nenhuma curva em particular, nem mesmo para o oscilador quártico, em contraste com o caso dos estados estacionários. É claro que quando $J=0$ o estado GK se reduz ao estado fundamental, e as distribuições de quase-probabilidade foram analisadas no apêndice.

Na figura 4.7(a) mostramos a densidade de probabilidade para um estado GK em $t=0$ no regime de alta energia, $J=500$. O valor de $\beta$ é claramente irrelevante nesse caso. Esse estado altamente não-local com diversos máximos e mínimos consiste na superposição de aproximadamente 70 estados estacionários em torno de $\langle n\rangle \approx 80$. Alguns instantes depois, (Figure 4.7(b)), entretanto, podemos ver o surgimento de dois pacotes de mínima incerteza centrados simetricamente em torno da origem, indicando que deve ser possível encontrar uma forma analítica simples para os estados GK no limite assintótico. De fato, nesse regime o comportamento do estados GK é inesperadamente bem descrito pela 

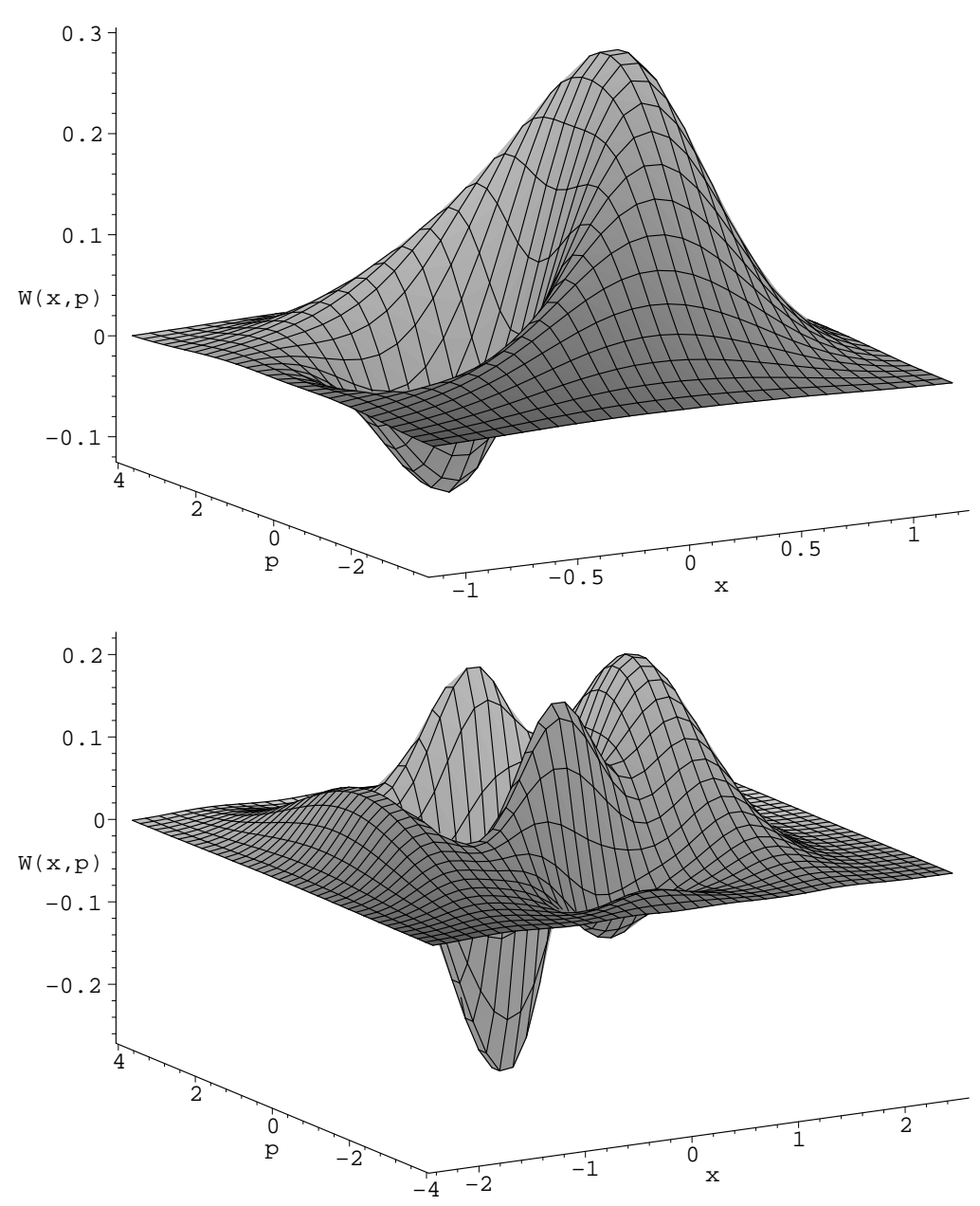

Figura 4.6: As funções de Wigner para estados GK com $J=1$ e $\beta=0$ (acima) e $\beta=10$ (abaixo). Ambas são negativas em regiões do espaço de fase, indicando o caráter não-clássico do estado.

expressão

$$
\langle x \mid J, t\rangle \simeq \frac{e^{i \theta(x, t)}}{\mathcal{N}}\left[e^{i \pi / 4} \Psi_{\alpha}(x, t)+e^{-i \pi / 4} \Psi_{-\alpha}(x, t)\right]
$$

onde $\mathcal{N}$ é uma constante de normalização e $\Psi_{\alpha}(x, t)$ é o estado coerente canônico (4.1) na representação de posição,

$$
\Psi_{\alpha}(x)=(\pi \sigma(t))^{-1 / 4} \exp \left\{-\frac{\left(x-x_{c l}(t)\right)^{2}}{2 \sigma(t)}+i p_{c l}(t) x\right\}
$$

$\operatorname{com} \alpha=x_{c l}(t) / \sqrt{\sigma(t)}+i \sqrt{\sigma(t)} p_{c l}(t)$. As quantidades $x_{c l}(t)$ e $p_{c l}(t)$ são a posição e o momentum clássicos para um oscilador quártico com energia igual a $J$. A largura dependente do tempo $\sigma(t)$ e a fase local tipo-gauge $\theta(x, t)$ devem ser 


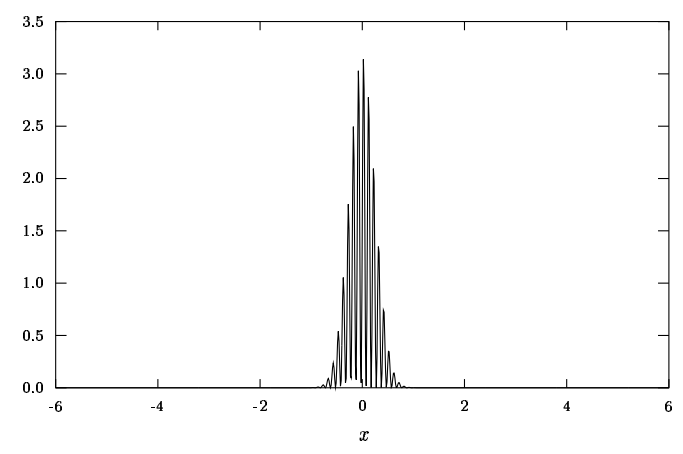

(a) $t=0$

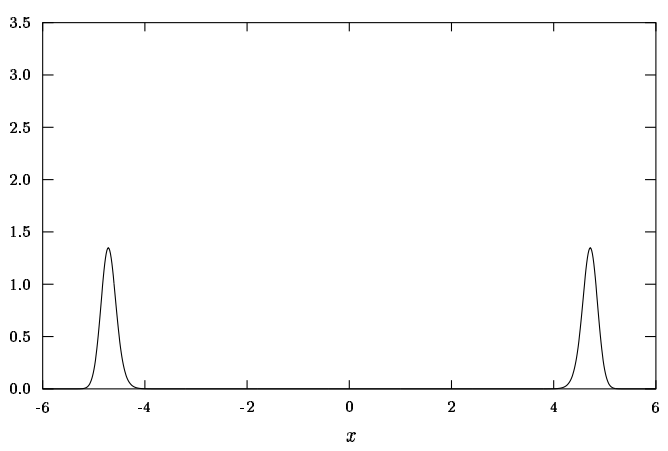

(c) $t=T / 4$

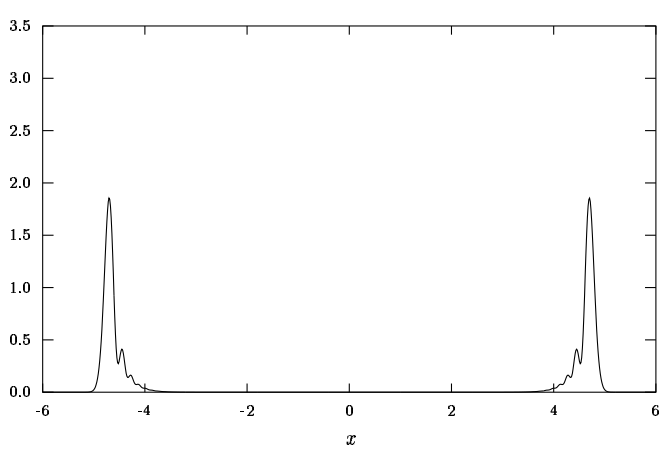

(e) $t=3 T / 4$

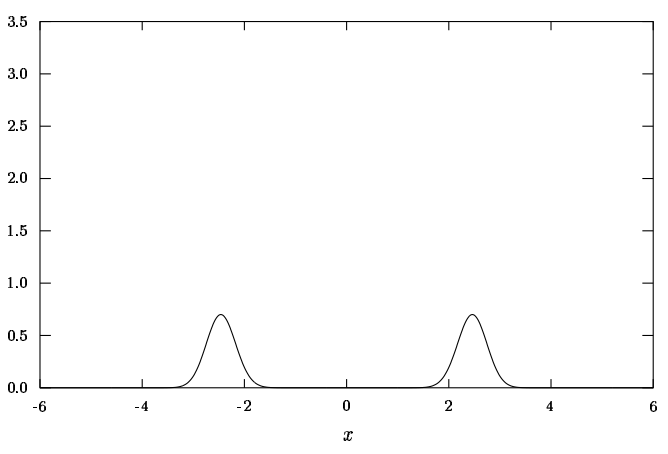

(b) $t=T / 10$

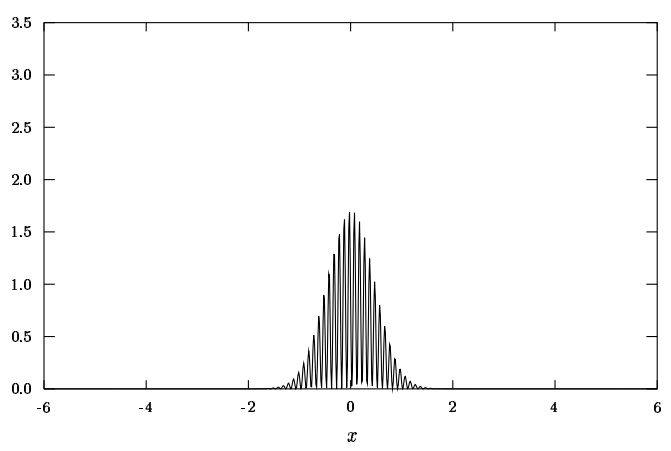

(d) $t=T / 2$

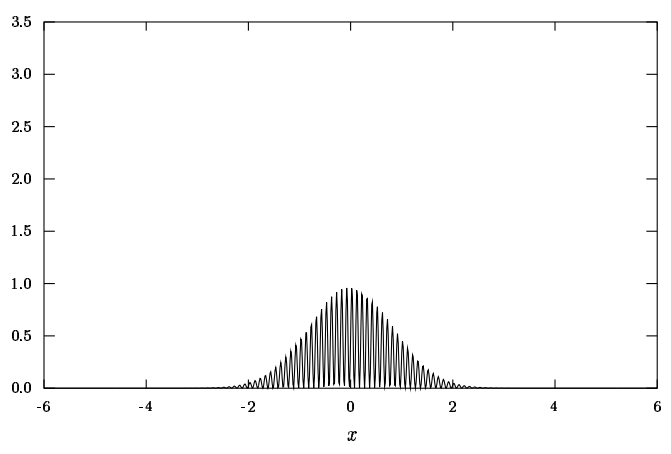

(f) $t=T$

Figura 4.7: A densidade de probabilidade para o estado GK com $J=500$, em diferentes frações do período clássico. Vemos a superposição coerente de dois estados de mínima incerteza.

escolhidas apropriadamente. A concordância entre essa expressão e os dados numéricos é geralmente em torno de 1\%, com concordância excepcional (uma parte por milhão) quando longe dos pontos de retorno.

Os estados de mínima incerteza (4.134) são famosos por serem o análogo quântico de uma partícula clássica e são considerados estados quânticos mesoscópicos quando $|\alpha|^{2} \gg 1$. Consequentemente, uma superposição de dois 
tais estados é usualmente interpretada como uma "superposição quântica macroscópica", uma entidade historicamente associada ao famoso "gato de Schrödinger" [69]. Esquemas para geração [70], detecção [5] e até teletransporte [71] de gatos de Schrödinger em cavidades têm sido objeto de intensas pesquisas nos últimos anos. O fato dos estados GK para um potencial $x^{4}$ serem desse tipo é realmente notável.

Na figura 4.7(c) as gaussianas estão sobre os pontos de retorno clássicos, e a interferência de meio-período é mostrada na figura 4.7(d). Esta função de onda é ortogonal àquela inicial. As gaussianas estão novamente sobre os pontos de retorno clássicos na figura 4.7(e), onde podemos notar o aparecimento de pequenas irregularidades responsáveis pelo maior desvio entre os dados numéricos e a aproximação (4.133). Finalmente mostramos na figura 4.7(f) a densidade de probabilidade depois de um período clássico. Ela está em fase com o estado inicial e a superposição dos dois é da ordem de $60 \%$.

As propriedades de reconstrução dos estados GK durante a evolução temporal podem ser analisadas usando-se a função de autocorrelação, $\mathcal{A}(t)=|\langle J, 0 \mid J, t\rangle|^{2}$, como função da energia de excitação $J$ e do tempo. O comportamento típico para baixas energias aparece na figura 4.8 para $\beta=10$, que fornece $J_{c} \simeq 4.14$. Cada um dos diversos máximos corresponde a uma reconstrução local do estado inicial, e todos os primeiros picos ocorrem, com boa aproximação, em múltiplos de um "quase-período" quântico $\tau$. Um máximo global é alcançado em torno de $T_{r}=11.3$, e vemos que duas escalas de tempo caracterizam o comportamento do sistema nesse ponto. A primeira, $\tau$, é análoga ao período clássico, embora em geral seja diferente dele. A segunda, o tempo de revival $T_{r}$, é cerca de uma ordem de magnitude maior e não está relacionado a propriedades clássicas. Outras escalas de tempo (por exemplo, tempo de super-revival) se tornam importantes para tempos longos, mas vamos restringir nossa atenção para tempos curtos.

A figura 4.8(b) mostra a função de autocorrelação para o mesmo potencial 


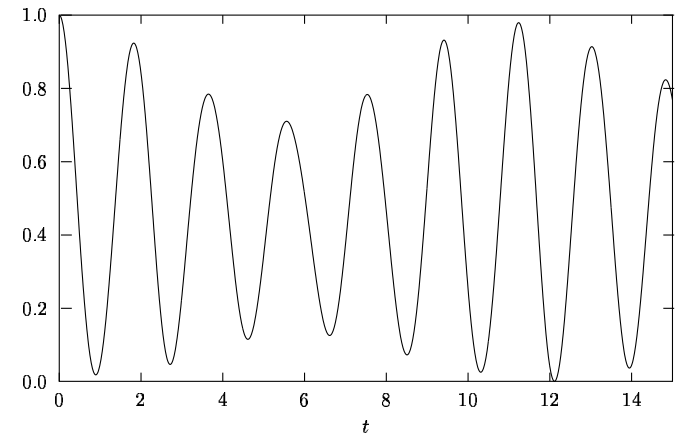

(a) $J=1$

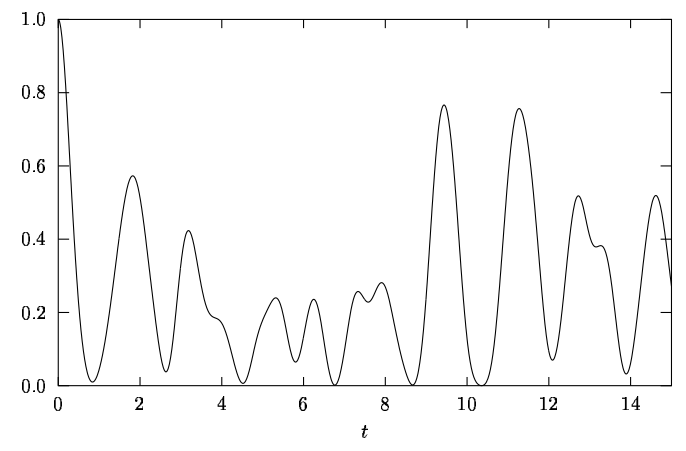

(c) $J=3$

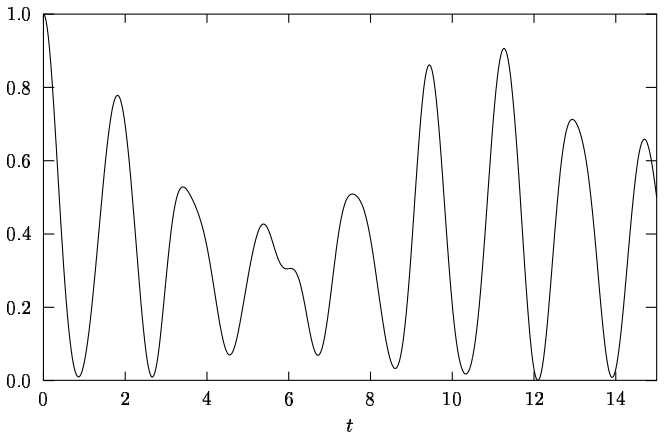

(b) $J=2$

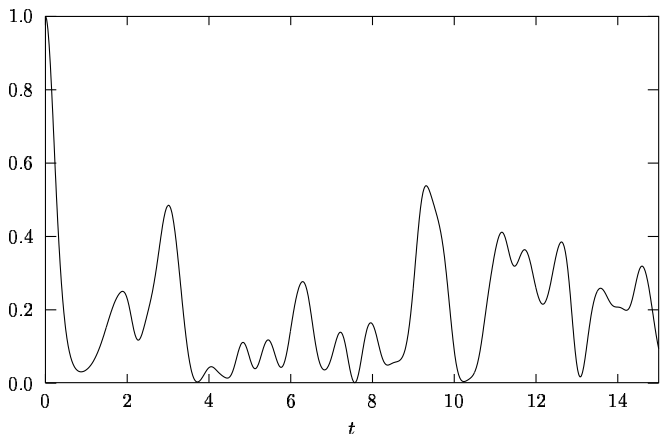

(d) $J=5$

Figura 4.8: A função de autocorrelação para $\beta=10$ e diferentes valores (pequenos) de $J$.

mas com o dobro da energia de excitação, $J=2$. A posição dos máximos muda moderadamente mas o comportamento do terceiro pico indica um processo que é central no entendimento da dinâmica dos estados GK sob mudanças na energia: o desdobramento de singularidades (máximos). Vemos uma singularidade lateral sendo criada perto do terceiro pico, que evolui par picos gêmeos na figura 4.8(c): uma singularidade instável desdobrou-se em dois máximos e um mínimo em um processo bem conhecido na teoria de singularidades. A figura 4.8(c) cooresponde a um valor de $J$ menor que $J_{c}$ e a figura $4.8($ d) mostra a função de autocorrelação logo depois de vencermos esse limite $(J=5)$. A posição do primeiro máximo, que corresponde ao limite clássico para certos valores de $J$ e $\beta$, não é fortemente afetada pela barreira. A mesma conclusão é válida para a posição do tempo de revival.

Na figura 4.9 vemos alguns gráficos de $\mathcal{A}(t)$ para valores mais altos de $J$. 


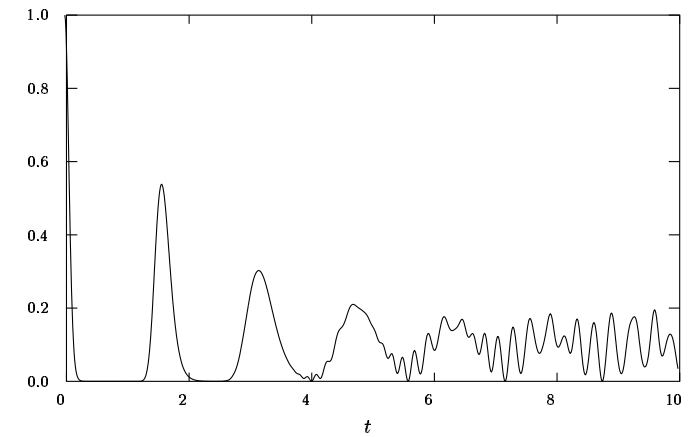

(a) $J=30$

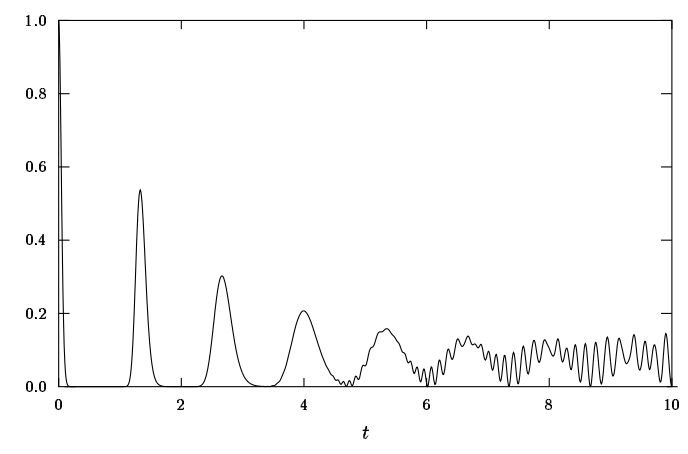

(c) $J=60$

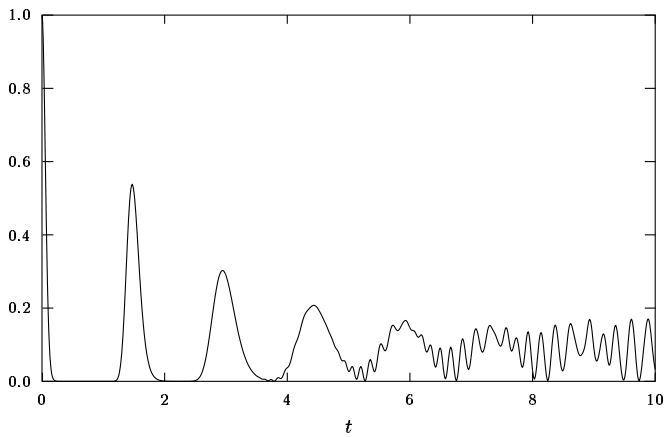

(b) $J=40$

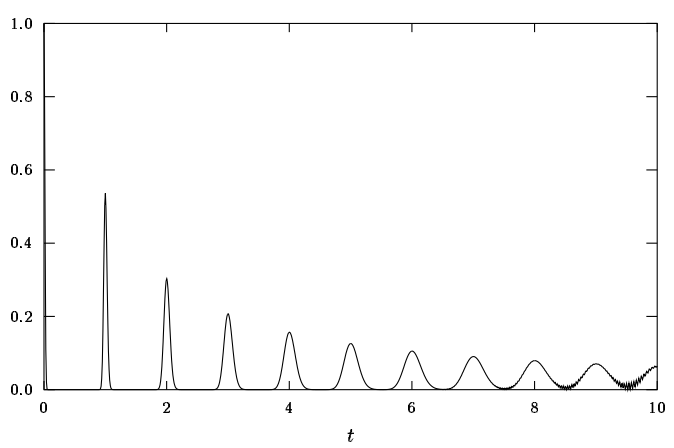

(d) $J=500$

Figura 4.9: A função de autocorrelação para valores maiores de $J$.

Concentremos nossa atenção no quarto pico. Ele não está bem resolvido na primeira figura, mas quando $J$ cresce notamos as pequenas singularidades que envolviam esse máximo serem suprimidas. Isso é confirmado nas figuras 4.9(c) e 4.9(d). Na última o pico é perfeitamente suave. A última figura corresponde a $J=500$, e está relacionada com a evolução do estado de gato (4.133). A reconstrução parcial ocorre em múltiplos do período clássico, mas as intensidades são progressivamente menores devido à dependência temporal do parâmetro $\sigma(t)$. Devemos notar que a regularidade para altas energias é diferente daquela para baixas energias, como podemos ver comparando as figuras 4.8(a) e 4.9(d): outro tipo de ordem é alcançado conforme a energia é aumentada.

Calculamos a autocorrelação para $\beta=20$ a fim de investigar a dependência em $\beta$ do quase-período $\tau$. Vimos que nesse caso $\tau$ é de fato o período clássico, em contraste com o caso $\beta=10$. Isso é explicado pelo número de estados es- 
tacionários abaixo da barreira em cada caso. Na figura 4.10 mostramos essa quantidade como função de $\beta$. A relação é aproximadamente linear e vemos que não há estadas "suficientes" quando $\beta=10$ para que o sistema se comporte de maneira "quase-clássica" abaixo da barreira. Por outro lado, sempre veremos o período clássico como escala de tempo relevante para energias altas, como notado no parágrafo anterior.

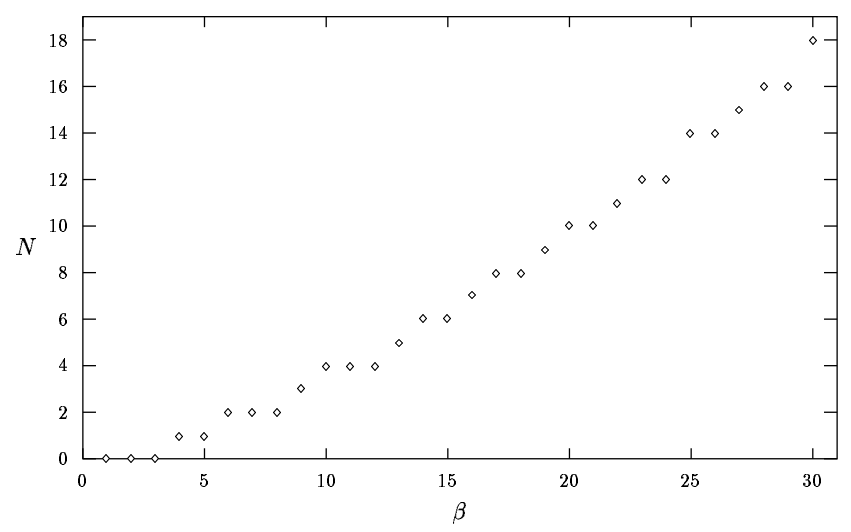

Figura 4.10: Número de estados abaixo da barreira como função de $\beta$.

Finalmente, mostramos na figura 4.11 a posição dos primeiros picos para $\beta=20$, como função de $J$, junto com o período clássico, $T_{c l}$, que é a linha sólida. Símbolos diferentes denotam diferentes intensidaes de reconstrução. Está claro que $\tau$ corresponde a $T_{c l}$ quando $J$ é muito pequeno comparado com a barreira. Conforme esse limite é cruzado o período clássico diverge, mas a posição do primeiro pico não é fortemente afetada. Sua intensidade, entretanto, é progressivamente diminuída até praticamente desaparecer. Uma bifurcação típica aparece na posição do segundo pico em torno de $J=12$, exibindo o desdobramento do singularidade. Antes dese ponto de bifurcação o estado inicial é parcialmente reproduzido em $T_{c l}$ e $2 T_{c l}$, e imediatamente após esse ponto dois máximos na reprodução da imagem (um "eco") são criados em um pequeno intervalo de tempo, um deles sendo dominante. Eles são ambos praticamente indiferentes ao cruzamento da barreira, mas não sobrevivem na região de altas energias. 


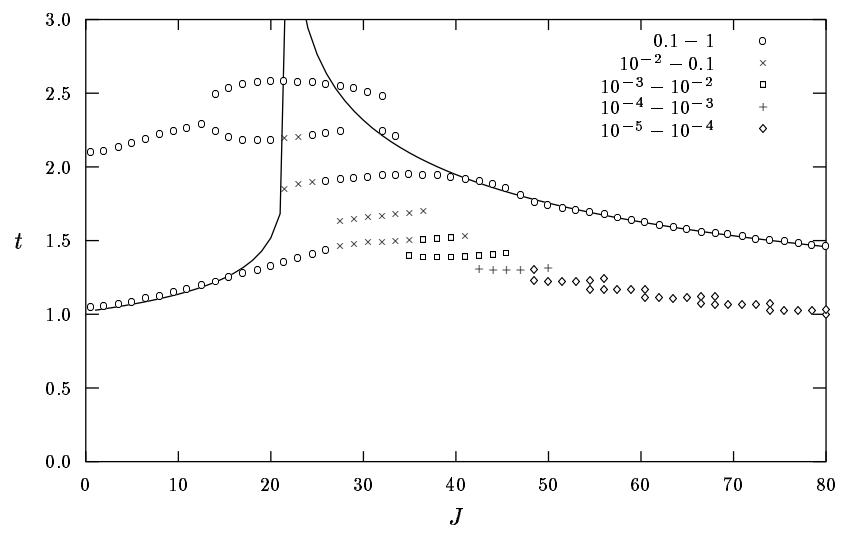

Figura 4.11: Picos iniciais da função de autocorrelação. Símbolos diferentes denotam intensidades diferentes, e a linha cheia é o período clássico $T_{c l}$. Note a concordância perfeita entre o primeiro pico e $T_{c l}$ quando $J$ está longe da barreira. 


\section{Capítulo 5}

\section{Conclusões}

Nossa contribuição para o estudo dos estados coerentes se deu em dois momentos. Primeiro, na apresentação detalhada e exaustiva do cálculo desse estados para um o grupo simplético $S p(4)$. As diversas definições e propriedades concebidas de forma abstrata puderam ser estudadas de forma analítica e numérica. Em segundo lugar, estendemos a teoria de Gazeau-Klauder e, além disso, a aplicamos a um potencial unidimensional que não possui soluções exatas.

Obtivemos explicitamente os estados coerentes para o grupo simplético unitário $S p(4)$ - tradicionalmente estudado por nosso grupo de pesquisa —, para representações irredutíveis arbitrárias. Encontramos sua normalização e a estrutura simplética correspondente, que junto com os valores médios dos operadores resulta em um limite clássico para sistemas quânticos com simetria simplética. No caso das representações simétricas obtivemos explicitamente a resolução da identidade e também coordenadas canônicas que trazem o parêntese de Poisson à sua forma tradicional.

Usamos esses estados coerentes para obter o limite clássico de um sistema quântico com simetria simplética que consiste de um termo integrável mais uma perturbação não-integrável. Vimos que a não-integrabilidade quântica — indicada pela distribuição de espaçamento de níveis dada pela distribuição de Wigner 
— está associada com um movimento caótico na representação de estados coerentes. Para pequenos valores da constante de acoplamento o espaço de fase exibe regiões regulares e caóticas, e simultaneamente a distribuição de espaçamento de níveis é bem descrita pela teoria de Berry-Robnik-Brody para sistemas de dinâmica mista no limite semi-clássico.

Para além da definição de estados coerentes a partir da perspectiva dos grupos de Lie, apresentamos o formalismo de estados coerentes generalizados desenvolvido por Gazeau e Klauder. Em um primeiro momento, estendemos esse formalismo a sistemas com mais de um grau de liberdade. Como ilustração dessa etapa, tratamos da termodinâmica de um gás bidimensional segundo as diferentes simetrias presentes. Também investigamos as propriedades desses estados no contexto do potencial de mínimos duplos unidimensional. A relação entre os regimes de baixa e alta energia foi considerado em termos do comportamento de singularidades na função de autocorrelação. Para altas energias vimos que estados de Gazeau-Klauder podem ser considerados Gatos de Schrödinger, já que consistem em uma superposição coerente de duas gaussianas de mínima incerteza. Esse foi um resultado bastante inesperado, que gerou uma reação positiva da comunidade.

As funções de Wigner e Husimi também foram objeto de estudo durante o trabalho. Obtivemos essas funções para vários estados estacionários do poço de mínimos duplos e investigamos suas propriedades de localização. A generalização dessas funções para outros grupos de Lie é um tópico importante, que pode se beneficiar dos cálculos apresentados aqui para o grupo simplético.

Acreditamos que diversas perspectivas interessantes de trabalho se abrem a partir das áreas apresentadas aqui. Mencionamos algumas possibilidades que nos parecem imediatas:

- Na aplicação dos estados coerentes do grupo $S p(4)$ em ótica quântica;

- Na abordagem algébrica do código genético, a partir de uma generalização 
para o grupo $S p(6)$;

- Em um estudo mais abrangente das conexões entre os estados coerentes e o caos quântico, possivelmente na formulação de propagadores semiclássicos;

- Na definição de funções de Wigner e de Husimi para o grupo simplético;

- Na aplicação dos estados de Gazeau-Klauder a outros potenciais de interesse, como o oscilador de Morse.

Esperamos que os progressos apresentados neste trabalho na compreensão dos estados coerentes e da simetria simplética possa contribuir na investigação dessas questões e de outras que envolvam álgebras de Lie ou assuntos correlatos. O entendimento dos estados coerentes e especialmente sua conexão com aproximações semiclássicas ainda possui um longo percurso a ser trilhado. 


\section{Apêndice A}

\section{Funções de Wigner e de Husimi}

Apresentamos neste apêndice a definição e as propriedades das funções de Wigner e de Husimi, e aplicamos esse formalismo ao caso do potencial de mínimos duplos. Funções de distribuição de quase-probabilidade (DQP), como as de Wigner e de Husimi, são ferramentas importantes no estudo da relação entre mecânica quântica e mecânica clássica e aspectos semiclássicos, uma vez que fornecem uma visão no espaço de fase da matriz densidade. Estados coerentes são centrais na formulação dessa teoria.

\section{A.1 Definição e propriedades}

Seja $|\psi\rangle$ o estado de um dado sistema quântico. Sua matriz densidade $\rho$ é o operador de projeção definido por $\rho=|\psi\rangle\langle\psi|$. A função de Wigner (FW) associada a uma matriz densidade é dada por [12]

$$
W(q, p)=\frac{1}{2 \pi \hbar} \int_{-\infty}^{\infty} d u \exp \left(\frac{i}{\hbar} p u\right)\langle q-u / 2|\rho| q+u / 2\rangle
$$

ou, em termos da função de onda, por

$$
W(q, p)=\frac{1}{2 \pi \hbar} \int_{-\infty}^{\infty} d u \exp \left(\frac{i}{\hbar} p u\right) \psi(q-u / 2) \psi^{*}(q+u / 2) .
$$


As grandezas observáveis do sistema, como valor médio de operadores e probabilidades de transição, podem ser obtidas da função de Wigner,

$$
\begin{aligned}
\langle A\rangle & =\operatorname{Tr}(A \rho)=\int d q d p W(q, p) a(q, p), \\
|\langle\psi \mid \phi\rangle|^{2} & =\int d q d p W_{\psi}(q, p) W_{\phi}(q, p),
\end{aligned}
$$

(onde $a(q, p)$ é o símbolo de Weyl de $\hat{A}$ ) e portanto podemos considerar toda a informação fisicamente relevante de um sistema como contida nessa função. A impressão de que essa função está definida sobre uma espécie de espaço de fase quântico é reforçada pelas relações

$$
\int_{-\infty}^{\infty} d q W(q, p)=|\phi(p)|^{2}, \quad \int_{-\infty}^{\infty} d p W(q, p)=|\psi(q)|^{2},
$$

onde $\phi(p)$ é a função de onda na representação de momentum. Vemos que $W(q, p)$ possui propriedades semelhantes a uma distribuição de probabilidade clássica. Entretanto, ela pode possuir valores negativos, como vemos a partir de (A.3) no caso em que $\langle\psi \mid \phi\rangle=0$. Portanto, a função de Wigner não pode ser interpretada diretamente como representando uma distribuição de probabilidade quântica, o que não é nenhuma surpresa, uma vez que o princípio de incerteza garante que nenhuma partícula possui valores bem definidos de posição e momentum simultaneamente.

Ainda assim, pode-se mostrar que no limite $\hbar \rightarrow 0$ a função $W(q, p)$ reproduz a distribuição de probabilidade clássica, ou seja, ela torna-se uma função altamente concentrada ao longo da trajetória clássica [72]. Entretanto, fora dessa trajetória $W(q, p)$ apresenta comportamento fortemente oscilatório, sendo inclusive negativa. A fim de obter uma função isenta dessas propriedades, definimos 
a função de Husimi (FH) através de uma "suavização" da função de Wigner [13]:

$$
\mathcal{H}(q, p)=\frac{1}{\pi^{2} \hbar^{2}} \int d x d v W(x, v) e^{-(q-x)^{2} / b^{2}-(p-v)^{2} b^{2} / \hbar^{2}} .
$$

Note que suavizamos $W(q, p)$ convoluíndo-a com funções gaussianas em $q$ e em $p$. A largura média dessas gaussianas satisfaz $\Delta q \Delta p=\hbar / 2$, ou seja, elas possuem mínima incerteza. Em outras palavras, através dessa suavização estamos introduzindo o princípio da incerteza na teoria, considerando que toda partícula "ocupa" uma região nesse espaço de fase quântico de área maior ou igual a $\hbar / 2$. $\mathrm{O}$ parâmetro $b$ controla a relação entre a largura em $q$ e a largura em $p$. É fácil ver que conforme esse parêmetro varia entre 0 e $\infty$ a função $\mathcal{H}(q, p)$ vai de "tipo-posição" a "tipo-momentum":

$$
\begin{aligned}
& \mathcal{H}(q, p)=\sqrt{\frac{2}{\pi}} \frac{b}{\hbar}|\psi(q)|^{2} e^{-2 b^{2} p^{2} / \hbar^{2}}, \quad b \ll 1 \\
& \mathcal{H}(q, p)=\frac{1}{b \sqrt{2 \pi}}|\phi(p)|^{2} e^{-q^{2} / 2 b^{2}}, \quad b \gg 1
\end{aligned}
$$

A função de Husimi é positiva definida, porém não possui as distribuições marginais de probabilidade dadas em (A.3). No limite $\hbar \rightarrow 0$ essa função também se concentra sobre a trajetória clássica, porém de forma mais bem comportada que a função de Wigner [73]. Em sistemas realmente quânticos, nos quais a constante de Planck $\hbar$ não pode ser considerada desprezível (e a energia de ponto zero deve ser levada em conta), vestígios da trajetória clássica são em geral chamados de "cicatrizes" [74]. Cicatrizes são em geral analisadas na distribuição de probabilidade, mas foram consideradas na FW e na FH por exemplo em [75].

Como já sabemos, os estados coerentes do oscilador harmônico, $|\alpha\rangle$, são dados por gaussianas de mínima incerteza. De fato, é fácil ver que a função de Husimi 
está intimamente ligada a esses estados:

$$
\mathcal{H}(q, p)=\frac{1}{\pi^{2} \hbar^{2}}\langle\alpha|\rho| \alpha\rangle=\frac{1}{\pi^{2} \hbar^{2}}|\langle\alpha \mid \psi\rangle|^{2}
$$

Os estados coerentes são a ferramenta fundamental utilizada em propostas recentes de se generalizar essas funções para estruturas algébricas mais elaboradas.

É possível unificar as funções de Wigner e de Husimi no mesmo formalismo, da seguinte maneira. Se definirmos, dado um operador $A$, uma função $F_{A}(q, p ; s)$ dada por

$$
\begin{aligned}
F_{A}(q, p ; s) & =\operatorname{Tr}[A \Delta(q, p ; s)] \\
\Delta(q, p ; s) & =\int \frac{d u d v}{2 \pi \hbar} \exp \left\{\frac{i}{\hbar}[u(q-Q)+v(p-P)]+s\left(u^{2}+v^{2}\right) / 4\right\}
\end{aligned}
$$

onde $Q$ e $P$ são os operadores posição e momentum, veremos que se $A=|\psi\rangle\langle\psi|$ obtemos, para $s=0$, a função de Wigner, e para $s=-1$ a função de Husimi. Uma definição mais geral para $\Delta$, que permite a construção de funções de Wigner para grupos de Lie gerais, foi apresentada em [15] e é baseada nos estados coerentes.

\section{A.2 Exemplo: Oscilador Harmônico}

Obteremos aqui as funções de Wigner e de Husimi para os estados estacionários do oscilador harmônico. Vamos supor $m=\omega=\hbar=1$ por simplicidade, onde $\omega$ é a frequencia natural de oscilação. Pela definição de $W(q, p)$, temos

$$
W_{n}(q, p)=\frac{1}{\pi \sqrt{\pi}} \frac{e^{-q^{2}}}{2^{n} n !} \int d y H_{n}(q-y) H_{n}(q+y) e^{-y^{2}+2 i p y}
$$

Usando o fato que

$$
\int d t e^{-t^{2}} H_{n}(t+q+\beta) H_{n}(t-q+\beta)=2^{n} n ! \sqrt{\pi} L_{n}\left(2\left(q^{2}-\beta^{2}\right)\right)
$$


e $H_{n}(-q)=(-1)^{n} H_{n}(q)$, temos

$$
W_{n}(q, p)=\frac{(-1)^{n}}{\pi} e^{-\left(q^{2}+p^{2}\right)} L_{n}\left(2\left(q^{2}+p^{2}\right)\right) .
$$

Notamos que a função de Wigner depende apenas de $q^{2}+p^{2}$, sendo portanto constante sobre as trajetórias clássicas. Notamos também que ela possui caráter oscilatório, sendo negativa em algumas regiões do espaco de fase.

A função de Husimi é muito mais simples de ser calculada neste caso, uma vez que sabemos que o estado coerente é dado por

$$
|\alpha\rangle=e^{-\left(q^{2}+p^{2}\right) / 2} \sum_{n=0}^{\infty} \frac{(q+i p)^{n}}{\sqrt{n !}}|n\rangle
$$

e portanto

$$
\mathcal{H}(q, p)=\frac{e^{-\left(q^{2}+p^{2}\right)}}{\pi^{2} \hbar^{2}} \frac{\left(q^{2}+p^{2}\right)^{n}}{n !}
$$

Vemos que novamente temos uma função constante sobre as trajetórias clássicas. Esse fenômeno não possui generalidade, estando restrito ao caso particular do oscilador harmônico. Queremos notar que essa função possui um máximo em $q^{2}+p^{2}=n$, ou seja, sobre a trajetória que possui a mesma energia do estado quântico. Esse é um caso trivial de cicatriz.

\section{A.3 O potencial de mínimos duplos}

Sistemas unidimensionais, como o oscilador de Morse [76] e o potencial de Pöschl-Teller [77], fornecem bons modelos para o estudo das propriedades de distribuições de quase-probabilidade (DQP). Nesta seção apresentamos as funções de Wigner e de Husimi associadas com os estados estacionários do poço duplo, dando especial atenção à estrutura clássica correspondente. Esse sistema tem a característica peculiar que os estados de baixa energia são bastante não-clássicos, 
devido ao efeito túnel, e esse fato se reflete nas DQP. Além disso, o poço duplo unidimensional foi usado como modelo no estudo de caos quântico e problemas correlatos $[60,78,79]$, e a análise de suas DQP pode trazer novos insights a esses assuntos.

Está claro a partir dos resultados (A.7) que a função de Husimi nem sempre possui cicatrizes, mas que essa propriedade deve depender de $b$ (veja também [80]). A gaussiana que aparece em (A.6) é muitas vezes interpretada como a função de onda do estado coerente de um oscilador harmônico, e nesse caso $b$ é naturalmente tomado igual a $\sqrt{\hbar /(2 m \omega)}$, onde $m$ e $\omega$ são a massa e a frequência angular do oscilador. Para um sistema geral, o parâmetro $b$ é muitas vezes tomado igual a 1, mas no estudo de efeitos de cicatriz acreditamos que uma escolha mais natural seja tomar como valor de $\omega$ a frequência angular associada à trajetória clássica relevante. Adotamos aqui, assim como em [81], essa prescrição.

A equação estacionária de Schrödinger para esse sistema pode ser escrita, em unidades adimensionais, como

$$
\left[-\frac{1}{2} \frac{d^{2}}{d x^{2}}-\frac{\beta}{2} x^{2}+x^{4}\right] \Psi_{n}(x)=e_{n} \Psi_{n}(x),
$$

onde o parâmetro $\beta$ controla a altura da barreira. Note que ao valor $\beta=0$ corresponde o oscilador quártico puro, e que no caso geral a altura é $\beta^{2} / 16$. A solução clássica desse potencial foi apresentada no Capítulo 4, e o período do movimento (que usamos para fixar o valor do parâmetro b), é

$$
T=\left\{\begin{array}{l}
\frac{2}{\sqrt{\lambda}} K\left(\sqrt{\frac{\lambda+\beta / 4}{2 \lambda}}\right), \quad \text { para } E \geq 0, \\
\sqrt{\frac{2}{\lambda+\beta / 4}} K\left(\sqrt{\frac{2 \lambda}{\lambda+\beta / 4}}\right), \quad \text { para } E<0,
\end{array}\right.
$$

onde $\lambda=\left(E+\beta^{2} / 16\right)^{1 / 4}$ e $K(\kappa)$ é a função elíptica completa do primeiro tipo. Vamos denominar o caso $E<0$ como movimento "confinado". 
Na figura 4.2 vemos os primeiros níveis de energia e correspondentes autofunções, tanto para o potencial quártico $(\beta=0)$ quanto para $\beta=10$. Notamos que o estado fundamental está centrado na origem no primeiro caso, mas isso não é mais verdade na presença da barreira, devido ao efeito túnel. Essa característica fortemente não-clássica está refletida em sua função de Wigner.
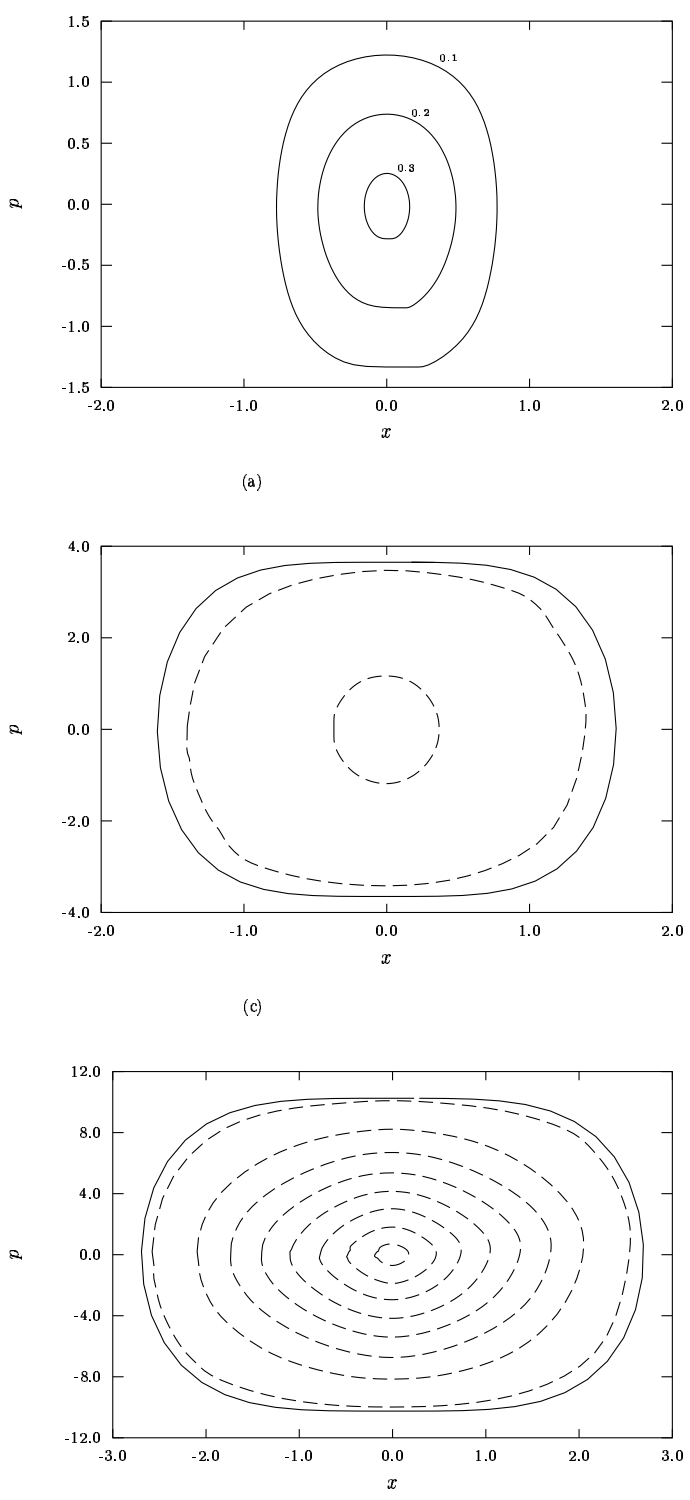
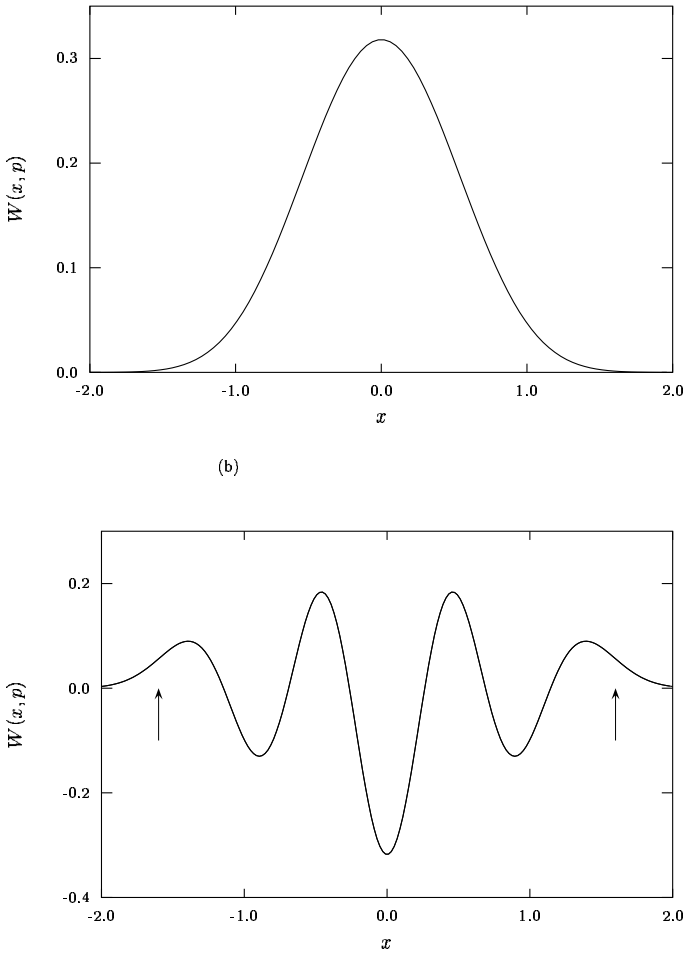

(d)

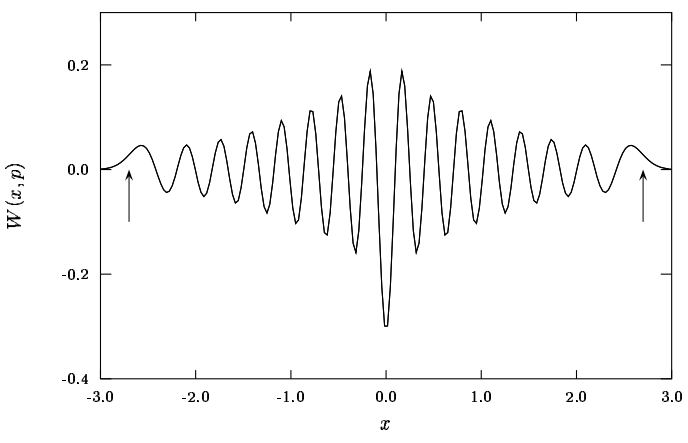

(f)

Figura A.1: FWs para $\beta=0$. (a) e (b) mostram curvas de nível e um corte através do plano $p=0$ da FW do estado fundamental. Em (c) e (e) as curvas tracejadas representam as curvas ao longo das quais a FW é um máximo local e a curva cheia é a trajetória clássica. (d) e (f) também são cortes através do plano $p=0$, e as setas indicam os pontos de retorno. 
Nas figuras A.1(a)-(b) vemos curvas de nível e um corte através do plano $p=0$ da FW do estado fundamental do oscilador quártico. Devemos notar que, como no caso do oscilador harmônico, essa função é positiva definida, em contraste com aquelas correspondentes a estados excitados, que aparecem nas figuras A.1(c)-(f) para $n=3$ e $n=15$. A linha cheia em (c) e (e) é a trajetória clássica com energia $e_{n}-e_{0}$ (note o papel da energia de ponto-zero; ela é necessária para garantir que ao estado fundamental corresponda uma partícula clássica em repouso), as setas mostram os pontos de retorno, e as linhas pontilhadas são as curvas ao longo das quais a função de Wigner é um máximo local. O comportamento fortemente oscilatório é típico da FW. Os estados estacionários apresentados aqui não são gerados por WKB, e portanto não é surpresa que o pico mais externo da FW e a trajetória clássica não coincidam. Entretanto, quando comparamos as figuras A.1(c) e A.1(e) vemos que a diferença entre essas curvas diminui conforme $n$ aumenta, já que no regime de altas enrgias a aproximação WKB deve ser válida.

A figura A.2 é análoga à figura A.2 mas representa a FH. Usando os períodos clássicos correspondentes às energias $e_{3}-e_{0}$ e $e_{15}-e_{0}$, encontramos para $\mathrm{o}$ parâmetro $b$ os valores 0.429 e 0.331 , respectivamente. Vemos que as oscilações que estão presentes na FW desaparecem na FH. A curva ao longo da qual a FH é máxima, a cicatriz, está representada pela linha pontilhada e deve ser comparado, nas figuras Figures A.2(c) e A.2(e), com a trajetória clássica, a linha cheia. É importante notar, entretanto, que a FH em geral não é constante ao longo dessa curva.

Voltamos agora nossa atenção para os casos em que a barreira está presente, $\beta \neq 0$. O espaço de fase clássico do sistema apresenta duas regiões disjuntas para energias abaixo de $\beta^{2} / 16$, e o tunelamento quântico possível entre essa regiões torna os estados abaixo da barreira bastante não-locais. Nenhuma aproximação semiclássica será válida nesse regime fortemente quântico e portanto não há razão para esperarmos que a função de Wigner seja localizado sobre alguma curva em 


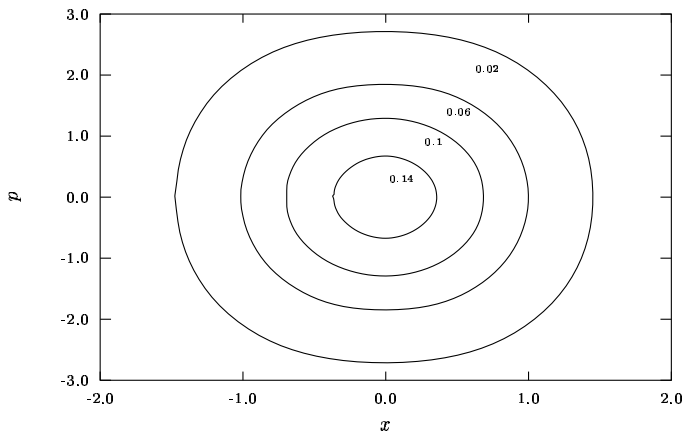

(a)
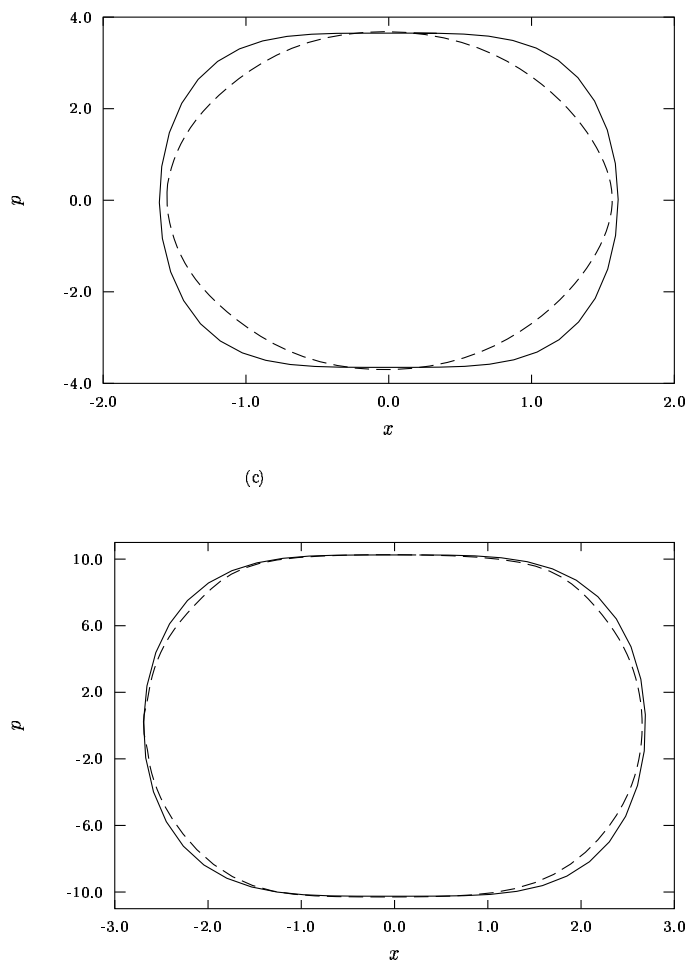

(e)

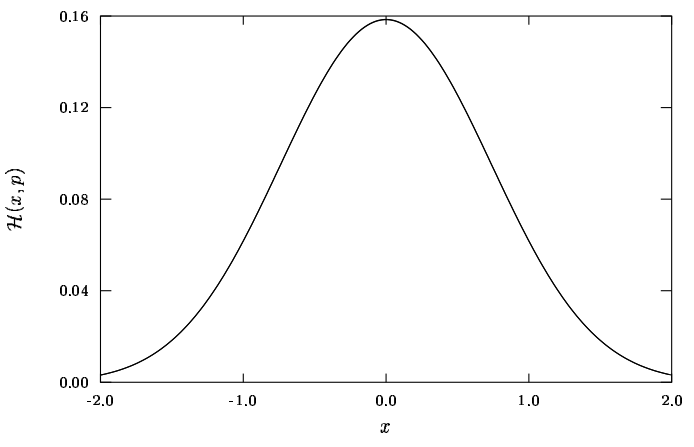

(b)

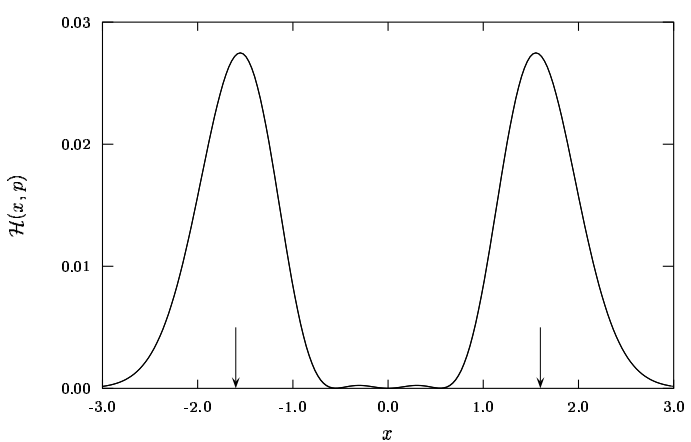

(d)

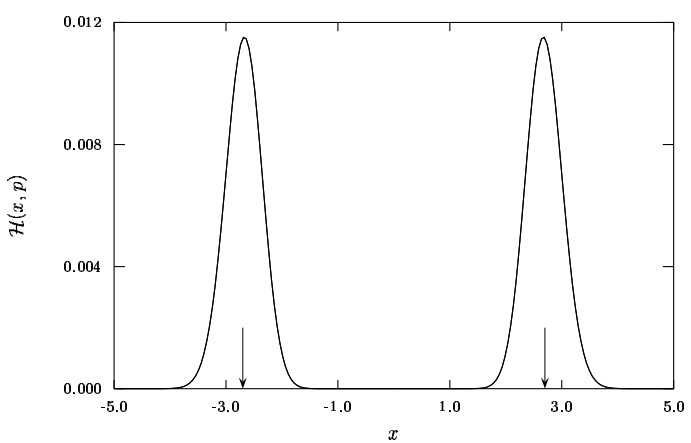

(f)

Figura A.2: FHs para $\beta=0$, em completa analogia com a figura anterior.

particular. Quando $\beta=10$ há 4 estadis abaixo da barreira, e na figura A.3 vemos a FW do estado fundamental. Além de ser negativa em algumas regiões do espaço de fase, essa função não possui qualquer propriedade de localização. As FWs para $n=1,2,3$ são também complexas, e o comportamento fortemente oscilatório está presente.

Se a energia estiver acima da barreira, entretanto, a situação é diferente. As regiões classicamente permitidas se comunicam e existe uma única trajetória 


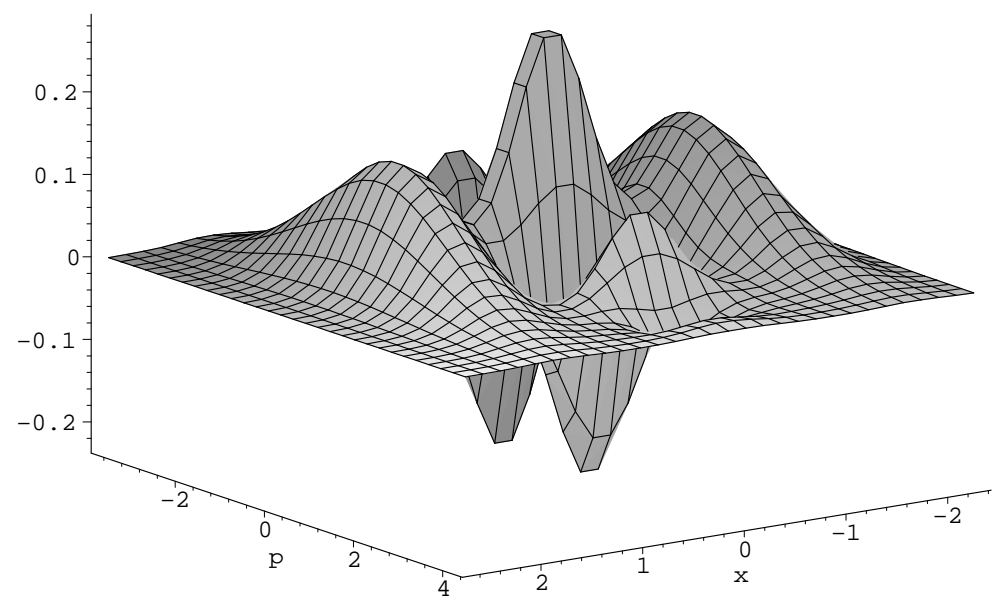

Figura A.3: $\mathrm{FW}$ do estado fundamental para $\beta=10$.

clássica. A função de Wigner então apresenta o mesmo tipo de estrutura que vimos na figura A.1 para o oscilador quártico, e seu pico mais externo possui uma aproximação assimtótica em direção a trajetória clássica, como vemos nas figuras A.4(a)-(b), que são análogas às figuras A.1(e)-(f) mas para $\beta=10$.

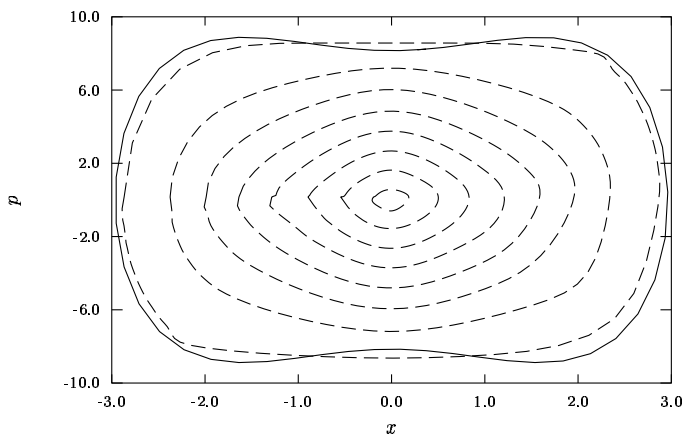

(a)

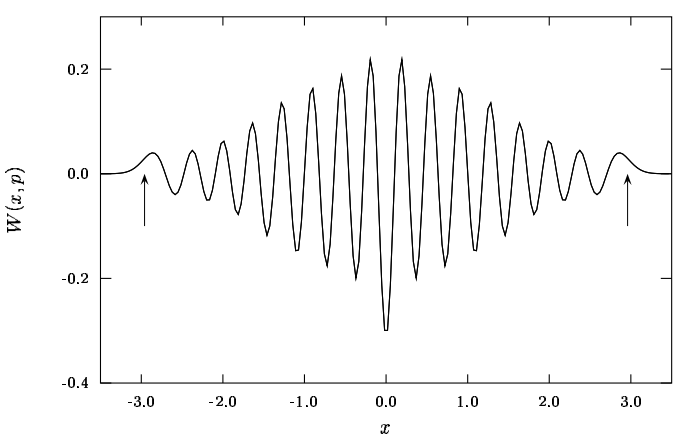

(b)

Figura A.4: FW para $n=15 \operatorname{com} \beta=10$.

A função de Husimi, por outro lado, é mais bem comportada: aquela correspondente ao estado fundamental tem dois picos (usamos $b=0.330$ nesse caso), um de cada lado do poço, como vemos nas figuras A.5(a)-(b). Quando isso é comparado com a complexidade da função de Wigner correspondente (figura A.3), fica claro que a FH está muito mais próxima da distribuição de probabilidade clássica. As figuras A.5(c)-(d) mostram cortes da FH para o terceiro estado exci- 
tado, $n=3$ (com $b=0.365)$. Esses cortes foram feitos, respectivamente, através do plano $p=0$ e através do plano definido por um dos pontos de equilíbrio, $x_{0}=1.58$ (novamente as setas indicam os pontos de retorno). Vemos que a $\mathrm{FH}$ pode tem uma clara cicatriz mesmo para estados abaixo da barreira, em contraste com a FW. De fato, existem duas cicatrizes, localizadas em torno de cada uma das duas possíveis órbitas clássicas.
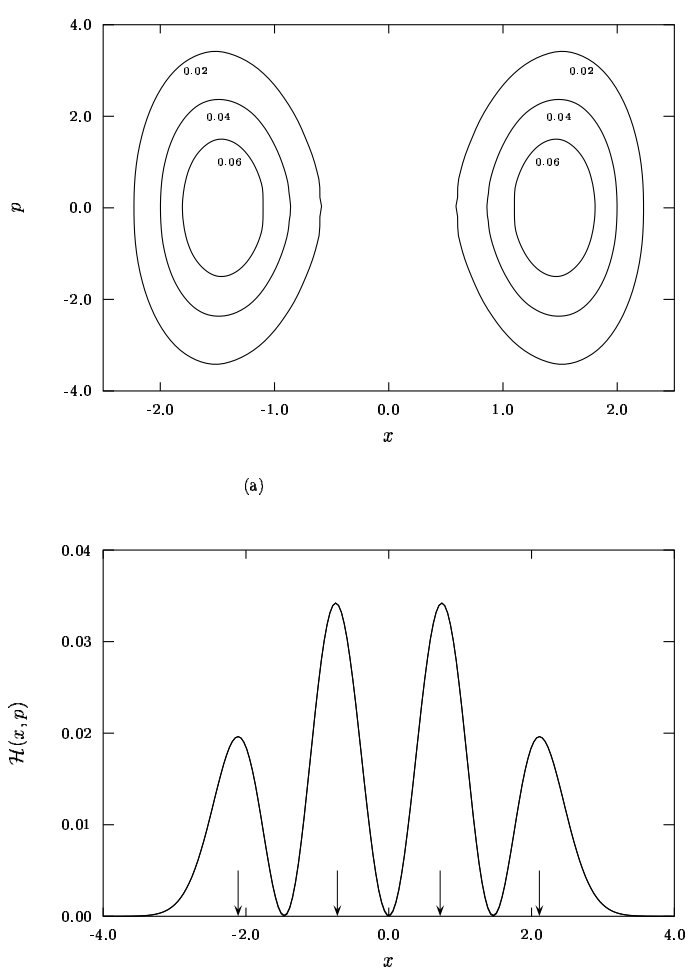

(c)

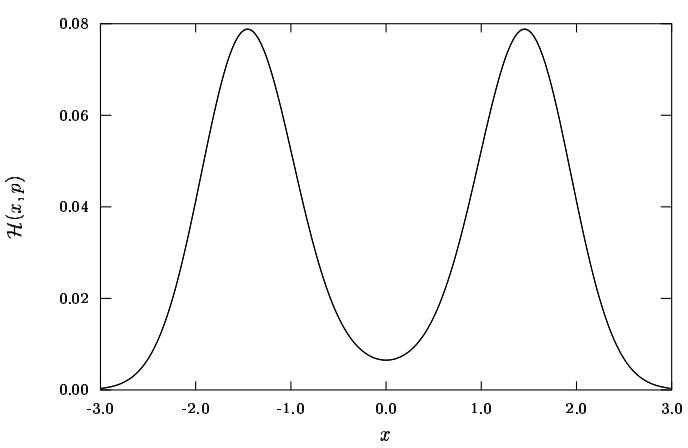

(b)

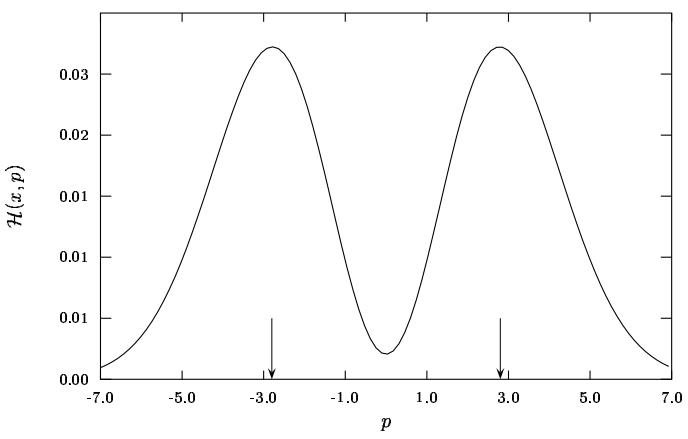

(d)

Figura A.5: FHs para $\beta=10$. (a) e (b) estão em analogia com a figura A.1. (c) é um corte através do plano $p=0$ e (d) é um corte através do plano $x=1.58$. Ambas correspondem a $n=3$.

Por outro lado, a FH pode apresentar um pico não-clássico na origem, como vemos na figura A.6(a), que é um corte através do plano $p=0$ para o estado $n=2(b=0.359)$. Poderíamos ser tentados a atribuir esse efeito como resultante da superposição das cicatrizes, mas isso não é possível em vista do caso $n=3$, onde as órbitas clássicas estão ainda mais próximas do que estão para $n=2$ mas ainda assim não se superpõem. Preferimos considerar esse pico não-clássico como 
uma indicação do confinamento, especialmente porque ele nunca está presente para $n \gg 1$. No regime de altas energias o efeito visual da cicatriz é mais impressionante, já que a função de Husimi tem apenas uma curva de pico, e esta se localiza muito próxima à trajetória clássica. Além disso, as rápidas oscilações que são típicas da função de Wigner estão novamente ausentes, como podemos ver nas figuras A.6(c)-(d), que correspondem ao estado $n=15$ (usando $b=0.363$ ).

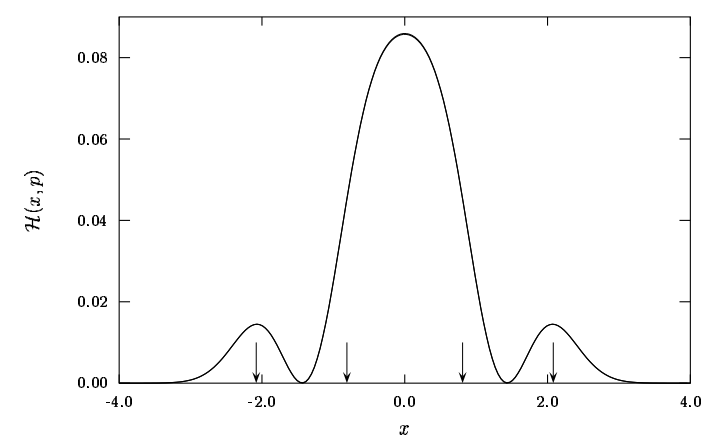

(a)

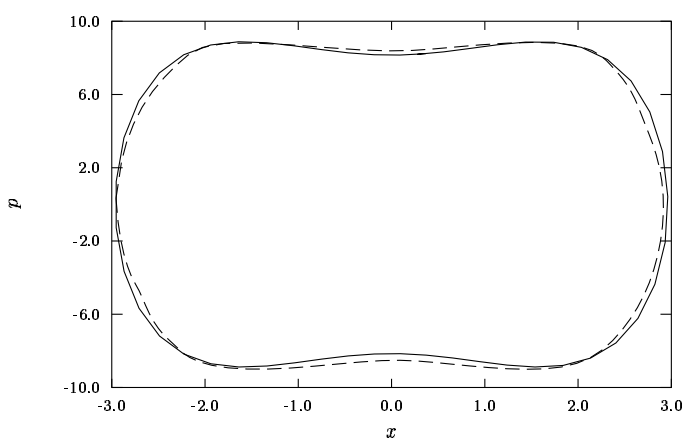

(c)

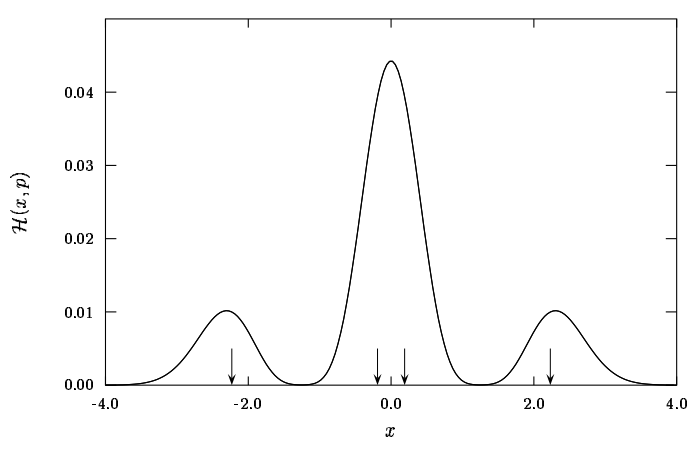

(b)

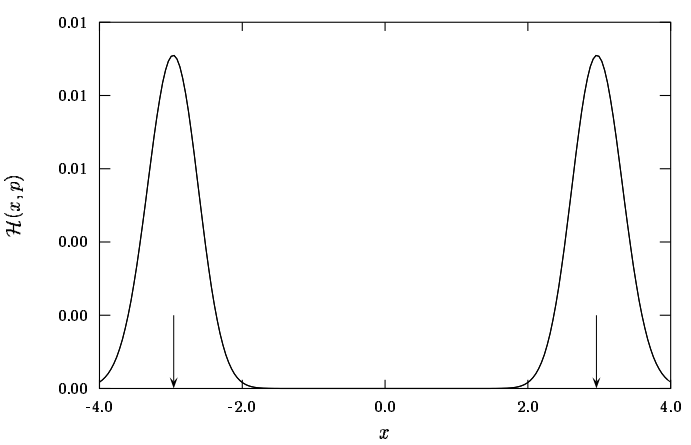

(d)

Figura A.6: FHs para $\beta=10$. (a) e (b) são cortes através do plano $p=0$, para $n=2$ e $n=4$ respectivamente. (c) e (d) são análogos à figura A.2(e) e (f).

Devemos notar que o quinto estado $n=4$ é bastante atípico. Embora esteja acima da barreira, ele está associado com um movimento clássico confinado, já que a diferença $e_{4}-e_{0}=6.06$ é menor que a altura da barreira 6.25. Este é um efeito inesperado da energia de ponto-zero, que pode aparecer apenas em regimes fortemente quânticos. Na figura A.6(b) vemos um corte através do plano $p=0$ de sua função de Husimi, que aparece na figura A.7 (o valor usado para b é 0.439), e o pico não-clássico na origem confirma o caráter confinado (embora não seja um 
máximo global como para $n=2$ ).

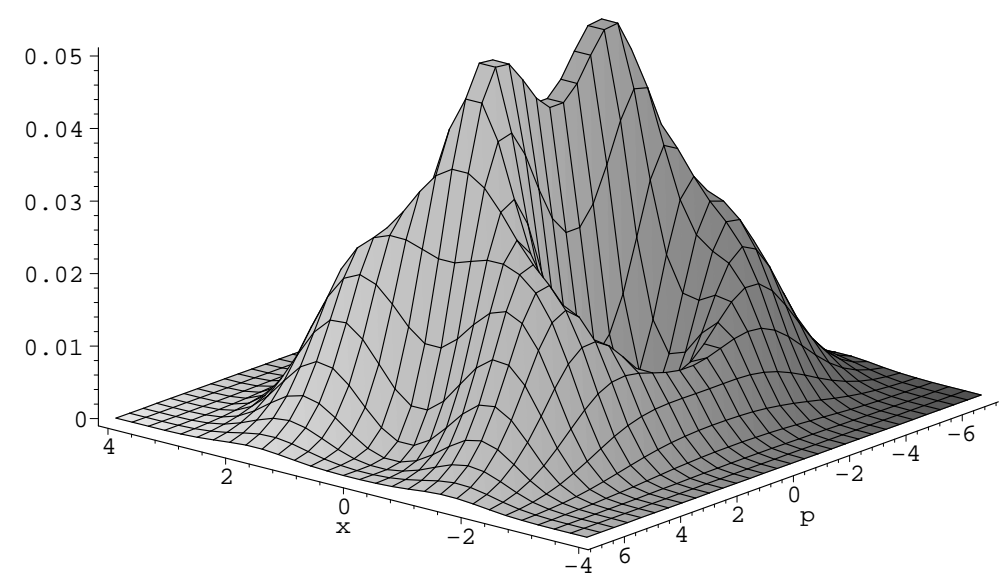

Figura A.7: FHs de $n=4$ para $\beta=10$.

Em resumo, apresentamos funções de Wigner e Husimi para os estados estacionários do oscilador quártico e o potencial mais geral de mínimos duplos. A rica estrutura desse sistema se reflete nos diferentes comportamentos exibidos por suas funções de distribuição de quase-probabilidade, que dependem qualitativamente da relação entre a energia do estado e a altura da barreira e podem exibir notáveis efeitos de cicatrizes. Uma vez que nossos cálculos não se baseiam em aproximações WKB, as funções de Wigner obtidas não são localizadas sobre a trajetória clássica, mas tendem a sê-lo para grandes números quânticos (com o comportamento oscilatório usual). A função de Husimi é mais próxima da distribuição de probabilidade clássica, já que possui propriedades de localização (com a escolha adequada do parâmetro b) mesmo para estados abaixo da barreira. A energia de ponto zero foi reconhecida como sendo importante quando se lida com estados de baixa energia. 


\section{Bibliografia}

[1] J.R. Klauder and B.S. Skagerstan (Eds.). Coherent States: Applications in physics and mathematical physics. World Scientific, 1985.

[2] M. O. Scully and M. S. Zubairy. Quantum optics. Cambridge University Press, 1997.

[3] J.M. Radcliff. Some properties of coherent spin states. J. Phys. A: Math. Gen. 4, 313, 1971.

[4] F.T. Arechi et all. Atomic coherent states in quantum optics. Phys. Rev. A 6, 2211, 1972.

[5] C.C. Gerry and R. Grobe. Cavity-QED state reduction method to produce atomic Schrödinger-cat states. Phys. Rev. A 57, 2247, 1998.

[6] A. Perelomov. Generalized coherent states and their applications. Springer, 1986.

[7] L.G. Yaffe. Large $N$ limits as classical mechanics. Rev. Mod. Phys. 54, 407, 1982.

[8] W.M. Zhang and D.H. Feng. Quantum nonintegrability in finite systems. Phys. Rep. 252, 1, 1995.

[9] E.H. Lieb. The classical limit of quantum spin systems. Commun. Math. Phys. 31, 615, 1973.

[10] D.M. Gitman and A. Shelepin. Coherent states of $S U(N)$ groups. J. Phys. A: Math. Gen. 26, 313, 1993; K. Nemoto. Generalized coherent states for $S U(N)$ systems. J. Phys. A: Math. Gen. 33, 3493, 2000.

[11] S. Gnutzmann and M. Kuś. Coherent states and the classical limit on irreducible $S U(3)$ representations. J. Phys. A: Math. Gen. 31, 9871, 1998.

[12] E.P. Wigner. On the quantum correction for thermodynamic equilibrium. Phys. Rev. 40, 749, 1932.

[13] K. Husimi. Some formal properties of the density matrix. Proc. Phys. Math. Soc. Jpn. 22, 264, 1940. 
[14] M. Hillery, R.F. O'Connel, M.O. Scully, and E.P. Wigner. Distribution functions in physics: fundamentals. Phys. Rep. 106, 121, 1984; H.W. Lee. Theory and application of the quantum phase-space distribution functions. Phys. Rep. 259, 147, 1995.

[15] C. Brif and A. Mann. Phase-space formulation of quantum mechanics and quantum-state reconstruction for physical systems with Lie-group symmetries. Phys. Rev. A 59, 97, 1999.

[16] J.R. Klauder. Continuous-representation theory. I. Postulates of continuousrepresentation theory, II. Generalized relation between quantum and classical dynamics. J. Math. Phys. 4, 1058, 1963.

[17] M. Baranger et all. Semiclassical approximations in phase space with coherent states. J. Phys. A: Math. Gen. 34, 7227, 2001.

[18] E.A. Kochetov. SU(2) coherent state path integral. J. Math. Phys. 36, 4667, 1995; M. Stone, K.S. Park, and A. Garg. The semiclassical propagator for spin coherent states. J. Math. Phys. 41, 8025, 2000.

[19] M. Pletyukhov and O. Zaitsev. Semiclassical theory of spin-orbit interaction in the extended phase space. J. Phys. A: Math. Gen. 36, 5181, 2003.

[20] A. Wünsche. Symplectic groups in quantum optics. J. Opt. B: Quantum Semiclass. Opt. 2, 73, 2000; A. Wünsche. The quantum-mechanical inhomogeneous symplecitc group. J. Opt. B: Quantum Semiclass. Opt. 4, 1, 2002.

[21] J.E.M. Hornos and Y.M.M. Hornos. Algebraic model for the evolution of the genetic code. Phys. Rev. Lett 71, 4401, 1993; J.E.M. Hornos, Y.M.M. Hornos, and M. Forger. Symmetry and symmetry breaking: An algebraic approach to the genetic code. Int. J. Mod. Phys. B 13, 2795, 1999.

[22] T. Prosen and M. Robnik. Semiclassical energy level statistics in the transition region between integrability and chaos: transition from Brody-like to Berry-Robnik behaviour. J. Phys. A: Math. Gen. 27, 8059, 1994.

[23] M. Robnik and T. Prosen. Comment on energy level statistics in the mixed regime. J. Phys. A: Math. Gen. 30, 8787, 1997.

[24] J.P. Gazeau and J.R. Klauder. Coherent states for systems with discrete and continuous spectrum. J. Phys. A: Math. Gen. 32, 123, 1999.

[25] J.R. Klauder. Coherent states for the hydrogen atom. J. Phys. A: Math. Gen. 29, L293, 1996.

[26] J.P. Antoine, J.P. Gazeau, P. Monceau, J.R. Klauder, and K. Penson. Temporally stable coherent states for infinite well and Pöschl-Teller potentials. J. Math. Phys. 42, 2349, 2001. 
[27] B. Roy and P. Roy. Gazeau-Klauder coherent states for the Morse potential and some of its properties. Phys. Lett. 296, 187, 2002.

[28] M. Novaes. Mecânica quântica no espaço de fase: II. Estados coerentes. Rev. Bras. Ens. Fís. 24, 437, 2002.

[29] M.G. Crawford. Temporally stable coherent states in energy-degerate systems: The hydrogen atom. Phys. Rev. A 62, 012104, 2000.

[30] R. Glauber. The quantum theory of optical coherence. Phys. Rev. 130, 2529, 1963; R. Glauber. Coherent and incoherent states of the radiation field. Phys. Rev. 131, 2766, 1963.

[31] E.C.G. Sudarshan. Equivalence of semiclassical and quantum mechanical descriptions of statistical light beams. Phys. Rev. Lett. 10, 277, 1963.

[32] M. A. Nielsen. Quantum computation and quantum information. Cambridge University Press, 2000.

[33] A.O. Barut and L Girardello. New coherent states associated with noncompact groups. Comm. Math. Phys. 21, 41, 1971.

[34] M.M. Nieto. Coherent states for general potentials I-VI. Phys. Rev. D 20, 1321, 1979.

[35] J.R. Klauder. Coherent state path integrals without resolutions of unity. Found. Phys. 31, 57, 2001.

[36] R. P. Feynman and A.R. Hibbs. Quantum mechanics and path integrals. McGraw-Hill, 1965.

[37] W. Lisiecki. Coherent state representations. A survey. Rep. Math. Phys. 35, 327, 1995.

[38] S.D. Bartlett, D.J. Rowe, and J. Repka. Vector coherent state representations, induced representations and geometric quantization: I. Scalar coherent state representations. J. Phys. A: Math. Gen. 35, 5599, 2002.

[39] R. Gilmore. Lie groups, Lie algebras and some of their applications. Krieger, 1994.

[40] W. Magnus. On the exponential solution of differential equations for a linear operator. Comm. Pure Appl. Math. 7, 649, 1954.

[41] V. Bargmann. On a hilbert space of analytic functions and an associated integral transform .1. Comm. Pure Appl. Math. 14, 187, 1961.

[42] N. Mukunda et all. Generalized coherent states and the diagonal representation for operators. J. Math. Phys. 44, 2479, 2003.

[43] S. Helgason. Differential geometry, Lie groups and symmetric spaces. Academic Press, 1978. 
[44] V.I. Arnold. Mathematical methods of classical mechanics. Springer, 1989.

[45] J.E. Marsden and T.S. Ratiu. Introduction to mechanics and symmetry. Springer, 1994.

[46] E.S. Bernardes. Matrix elements for the symplectic sp(4) Lie algebra. J. Phys. A: Math. Gen. 32, 6295, 1999.

[47] M. Cerkaski. Branching rules for $S p(2 n)$ algebra reduction on the chain $S p(2 n-2) \otimes S p(2)$. J. Math. Phys. 28, 989, 1987.

[48] M. Novaes and J.E.M. Hornos. Coherent states for the unitary symplectic group. em preparação .

[49] S. Gnutzmann, F. Haake, and M. Kuś. Quantum chaos of $S U(3)$ observables. J. Phys. A: Math. Gen. 33, 143, 2000.

[50] O. Bohigas, M.J. Giannoni, and C. Schmit. Chaotic movement and universality of spectral fluctuations. Phys. Rev. Lett. 52, 1, 1984.

[51] M.L. Mehta. Random Matrices. Academic Press, 1991.

[52] F. Haake. Quantum signatures of chaos. Springer, 2001.

[53] M.V. Berry and M. Robnik. Semiclassical level spacings when regular and chaotic orbits coexist. J. Phys. A: Math. Gen. 17, 2413, 1984.

[54] T.A. Brody. Statistical measure for repulsion of energy-levels. Lett. Nuovo Cimento 7, 482, 1973.

[55] M. Novaes and J.E.M. Hornos. Quantum nonintegrability and the classical limit for usp(4) systems. em preparação .

[56] A.H. El Kinani and M. Daoud. Generalized intelligent states for nonlinear oscillators. Int. J. Mod. Phys. B 15, 2465, 2001.

[57] J.M. Hollingworth, A. Konstadopoulou, S. Chountasis, A. Vourdas, and N.B. Backhouse. Gazeau-Klauder coherent states in one-mode systems with periodic potential. J. Phys. A: Math. Gen. 34, 9463, 2001.

[58] A.H. El Kinani and M. Daoud. Generalized coherent and intelligent states for exact solvable quantum systems. J. Math. Phys. 43, 714, 2002; A.H. El Kinani and M. Daoud. Generalized intelligent states for an arbitrary quantum system. J. Phys. A: Math. Gen. 34, 5373, 2001.

[59] M. Daoud and A.H. El Kinani. The Moyal bracket in the coherent states framework. J. Phys. A: Math. Gen. 35, 2639, 2002. 
[60] W.E. Bies, L. Kaplan, and E.J. Heller. Scarring effects on tunneling in chaotic double-well potentials. Phys. Rev. E 64, 016204, 2001; F. Grossmann, T. Dittrich, P. Jung, and P. Hänggi. Coherent destruction of tunneling. Phys. Rev. Lett. 67, 516, 1991; O. Bohigas, S. Tomsovic, and D. Ullmo. Manifestations of classical phase-space structures in quantum-mechanics. Phys. Rep. 223, 43, 1993; M. Grifoni and P. Hänggi. Driven quantum tunneling. Phys. Rep. 304, 229, 1998.

[61] L. Mandel. Sub-poissonian photon statistics in resonance fluorescence. Opt. Lett. 4, 205, 1979.

[62] F.A. Berezin. General concept of quantization. Commun. Math. Phys. 40, 153, 1975.

[63] M. Novaes and J.P. Gazeau. Multidimensional generalized coherent states. J. Phys. A: Math. Gen. 36, 199, 2003.

[64] J.P. Gazeau, P.Y. Hsiao, and A. Jellal. Exact trace formulas for twodimensional electron magnetism. Phys. Rev. B 65, 094427-1, 2002.

[65] M. Novaes, M.A.M. de Aguiar, and J.E.M. Hornos. Generalized coherent states for the double-well potential. J. Phys. A: Math. Gen. 36, 5773, 2003.

[66] P.F. Byrd and M.D. Friedman. Handbook of elliptic integrals for engineers and scientists. Springer, 1971.

[67] D.F. Walls. Squeezed states of light. Nature 306, 141, 1983.

[68] M.M. Nieto. Coherent states for unusual potentials. Mod. Phys. Lett. A 16, $2305,2001$.

[69] E. Schrödinger. Naturwissenschaften 23, 812, 1935.

[70] B. Yurke and D. Stoler. Generating quantum-mechanical superpositions of macroscopically distinguishable states via amplitude dispersion. Phys. Rev. Lett. 57, 13, 1986; M. Brune et all. Observing the progressive decoherence of the "meter" in a quantum measurement. Phys. Rev. Lett. 77, 4887, 1996.

[71] L. Davidovich, N. Zagury, M. Brune, J.M. Raimond, and S. Haroche. Teleportation of an atomic state between 2 cavities using nonlocal microwave fields. Phys. Rev. A 50, R895, 1994; M.H.Y. Moussa. Teleportation with identity interchange of quantum states. Phys. Rev. A 55, R3287, 1997; N.G. de Almeida, R. Napolitano, and M.H.Y. Moussa. Accuracy of a teleported cavity-field state. Phys. Rev. A 62, 010101, 2000.

[72] M.V. Berry. Semi-classical mechanics in phase space: a study of Wigner's function. Philos. Trans. R. Soc. London A 287, 237, 1977. 
[73] J.E. Harriman and M.E. Casida. Husimi representation for stationary states. Int. J. Quantum Chem. 45, 263, 1993; J. Kurchan, P. Leboeuf, and M. Saraceno. Semiclassical approximations in the coherent-state representation. Phys. Rev. A 40, 6800, 1989.

[74] E.J. Heller and S. Tomsovic. Postmodern quantum-mechanics. Phys. Today 46, 38, 1993.

[75] A.C. Oliveira and M.C. Nemes. Classical structures in the Husimi distributions of stationary states for $\mathrm{H}-2$ and $\mathrm{HCl}$ molecules in the Morse potential. Phys. Scripta 64, 279, 2001; F. Toscano, M.A.M. de Aguiar, and A.M. Ozorio de Almeida. Scars of the Wigner function. Phys. Rev. Lett. 86, 59, 2001; P.A. Dando and T.S. Montero. Quantum surfaces of section for the diamagnetic hydrogen-atom - Husimi functions versus Wigner functions. J. Phys. B: Atom. Mol. Opt. Phys. 27, 2681, 1994; K. Muller and D. Wintgen. Scars in wave-functions of the diamagnetic Kepler-problem. J. Phys. B: Atom. Mol. Opt. Phys. 27, 2693, 1994.

[76] A. Frank and K.B. Wolf A.L. Rivera. Wigner function of Morse potential eigenstates. Phys. Rev. A 61, 054102, 2000.

[77] G.W. Bund and M.C. Tijero. Mapping Wigner distribution functions into semiclassical distribution functions. Phys. Rev. A 61, 052114, 2000.

[78] R.E. de Carvalho and M.A.M. de Aguiar. Quantum and semiclassical Husimi distribuitions for a one dimensional ressonant system. Phys. Rev. A 46, 1128, 1992.

[79] O. Agam and S. Fishman. Quantum eigenfunctions in terms of periodicorbits of chaotic systems. J. Phys. A: Math. Gen. 26, 2113, 1993.

[80] M.A.M. de Aguiar and A.M. Ozório de Almeida. On the probability density interpretation of smoothed Wigner functions. J. Phys. A: Math. Gen. 23, L1025, 1990.

[81] M. Novaes. Wigner and Husimi functions in the double-well potential. $J$. Opt. B: Quantum Semiclass. Opt. 5, S342, 2003. 